\title{
MONITORING BIOFILM CO 2 PRODUCTION IN RESPONSE TO ANTIBIOTIC EXPOSURES
}

\author{
by \\ Lindsay Jackson \\ Bachelor of Biology and Medical Sciences, University of Western Ontario 2010
}

\author{
A dissertation \\ presented to Ryerson University \\ in partial fulfillment of the \\ requirements for the degree of \\ Doctor of Philosophy \\ in the program \\ of Molecular Science
}

Toronto, Ontario, Canada, 2015

(C) Lindsay Jackson 2015 


\section{AUTHOR'S DECLARATION}

I hereby declare that I am the sole author of this dissertation. This is a true copy of the dissertation, including any required final revisions, as accepted by my examiners.

I authorize Ryerson University to lend this dissertation to other institutions or individuals for the purpose of scholarly research

I further authorize Ryerson University to reproduce this dissertation by photocopying or by other means, in total or in part, at the request of other institutions or individuals for the purpose of scholarly research.

I understand that my dissertation may be made electronically available to the public. 


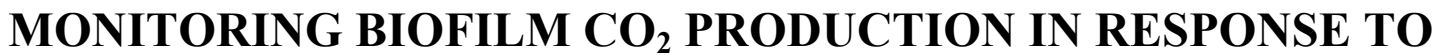 ANTIBIOTIC EXPOSURES
}

\author{
Lindsay Jackson, Doctor of Philosophy, Molecular Science \\ Ryerson University, 2015
}

\begin{abstract}
Bacteria commonly exist in nature as biofilms which help protect them from harsh environmental conditions, thus increasing their ability to adapt and survive. In clinical settings antibiotic treatments based on planktonic susceptibility tests are ineffective against biofilm infections, as biofilms can withstand much higher antibiotic concentrations. To further elucidate biofilm behaviour, multispecies and pure culture biofilms were grown in a $\mathrm{CO}_{2}$ evolution measurement system (CEMS) that tracks whole-biofilm $\mathrm{CO}_{2}$ production non-destructively and in real-time. In addition, metagenomic analysis was performed on multispecies cultures to monitor community dynamics and the intrinsic potential in different physiological states. Multispecies biofilms grown in CEMS were exposed to aminoglycosides under various environmental conditions (e.g. nutrient and $\mathrm{pH}$ variations). Multispecies biofilms were most susceptible to highdose streptomycin exposures when grown for less than 48 hours (young biofilm). However, carbon addition to the antibiotic medium decreased young biofilm susceptibility to streptomycin. Furthermore, young biofilms were susceptible to $4000 \mathrm{mg} / 1$ doses of streptomycin and gentamicin at pH 6.0 and to $4000 \mathrm{mg} / 1$ doses of streptomycin at pH 7.4. Biofilms were least susceptible to the aminoglycosides at neutral $\mathrm{pH}$. Metagenomic analysis on the multispecies biofilms revealed the presence of aminoglycoside-modifying enzymes and that the cultures contained Pseudomonas aeruginosa and Stenotrophomonas maltophilia strains. The dominant strain in planktonic cultures was $P$. aeruginosa. However, in complex growth medium, $S$. maltophilia increased in proportion upon biofilm development and became the dominant species following a high-dose streptomycin exposure. The monitoring of biofilm metabolic responses revealed adding carbon to the antibiotic medium or exposing the biofilms to aminoglycosides at neutral $\mathrm{pH}$ reduces biofilm aminoglycoside susceptibility. In addition, metagenomic analysis
\end{abstract}


uncovered community composition and genes that can play a role in biofilm survival to the antibiotics. However, even with the presence of antibiotic resistance genes, carbon and $\mathrm{pH}$ play an important role in biofilm survival to high-dose antibiotic exposures. 


\section{ACKNOWLEDGEMENTS}

I am very grateful to my Supervisor Dr. Gideon Wolfaardt who has shown me unwavering support and has always encouraged me to be self-reliant. I have truly enjoyed working for you as you have managed to provoke excitement in our research from across the world. To Dr. Otini Kroukamp, I want to thank you for all our inspiring discussions, your guidance, and support. My definition of learning has forever changed from having you as a mentor and I can only hope to spark interest in others to learn as you have for me. I would also like to thank my committee members Dr. Martina Hauser and Dr. Celine Levesque for your critical insights when my focus became too narrow. Dr. William Young has been invaluable in my learning and understanding of genetics, genomics, and the technology needed to be applied to this work.

I would also like to thank all of the Wolfaardt lab members for their friendship, support, humour, and help. I will always be grateful to each of you for the great moments we shared.

Lastly, thank you to my family for all your love and support. In particular, my parents and partner who have always taking an interest in what I am doing and helped me get through the tough times. 


\section{Contents}

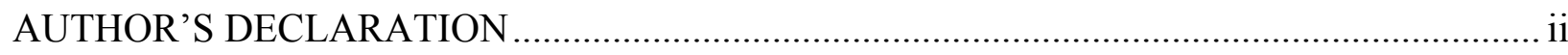

MONITORING BIOFILM CO $\mathrm{CO}_{2}$ PRODUCTION IN RESPONSE TO ANTIBIOTIC

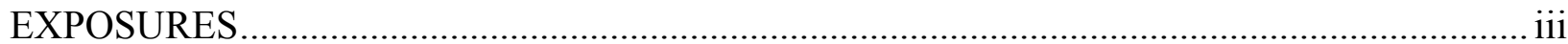

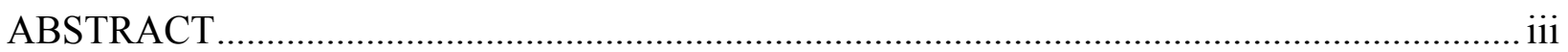

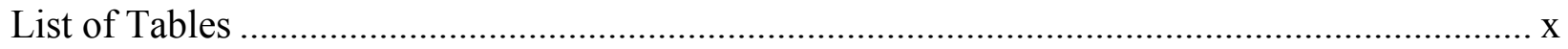

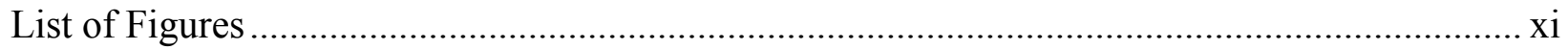

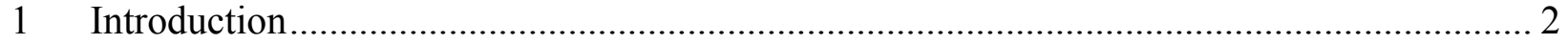

$1.1 \quad$ Biofilm Nature and Pathogenicity ......................................................................... 2

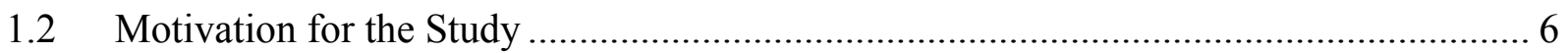

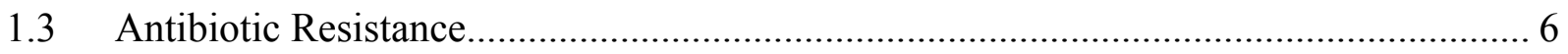

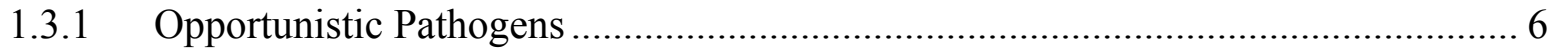

1.3.2 Antimicrobial Resistance Mechanisms in Pseudomonas aeruginosa ...................... 8

1.3.3 Antipseudomonal agents and antibiotics effective against Gram-negative bacteria 10

1.3.4 Streptomycin and Gentamicin Aminoglycoside Antibiotics .................................. 11

1.3.5 Applications for aminoglycosides in clinical and agricultural settings .................. 12

1.3.6 Application of fluoroquinolones with special reference to ciprofloxacin .............. 13

1.3.7 Antibiotic Susceptibility Testing: Bacterial Biofilm and Planktonic Systems:...... 13

1.3.8 Minimum Inhibitory Concentration (MIC) and Minimum Bacterial Eradication Concentration (MBEC) …………………………………........................................ 13

1.3.9 Real-Time Monitoring of Antimicrobial Effects on Biofilms ............................... 15

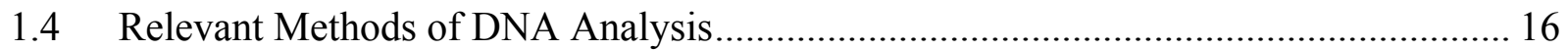

1.4.1 Polymerase Chain Reaction and Genomic Analysis............................................. 16

1.4.2 Overview of mg-RAST (metagenomic-Rapid Annotation using Subsystem

Technology) method of genome analysis ....................................................................... 17

$1.5 \quad$ Purpose of the Proposed Research Project.................................................................. 17

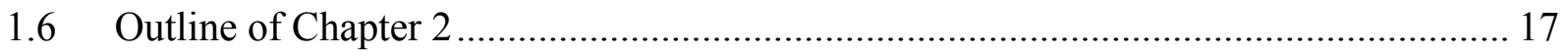

1.7 Outline of Chapter 3: The influence of $\mathrm{pH}$ whole-biofilm susceptibility to gentamicin and streptomycin as monitored by $\mathrm{CO}_{2}$ production ......................................................... 19

1.8 Outline of Chapter 4: The intrinsic potential of the planktonic and biofilm cultures used

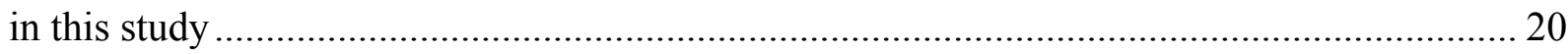

2 Biofilm-Metabolic Response to Streptomycin is Dependent upon Carbon Source and/or

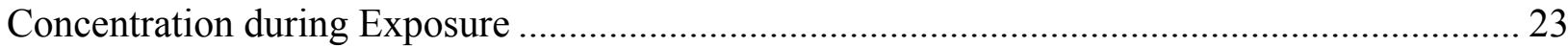




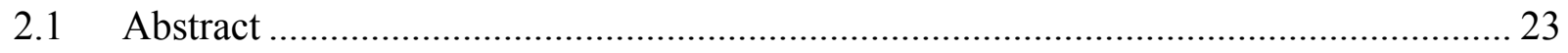

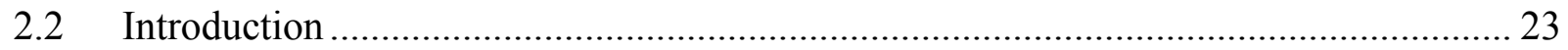

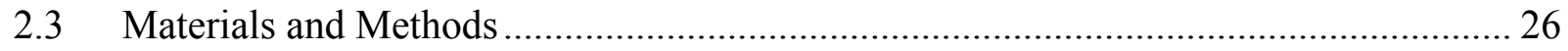

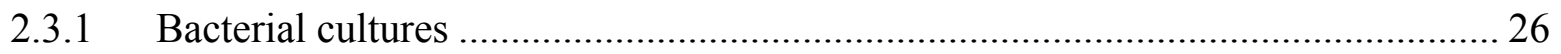

2.3.2 Antibiotic minimum inhibitory concentration (MIC) ……………………........ 27

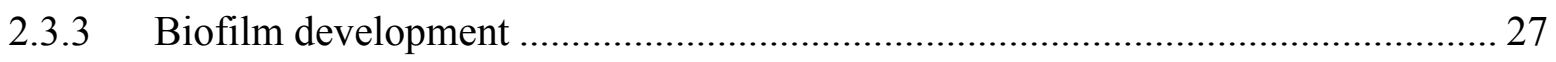

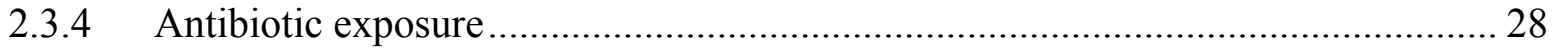

2.3.5 Viable cell counts in biofilm effluent ………….............................................. 28

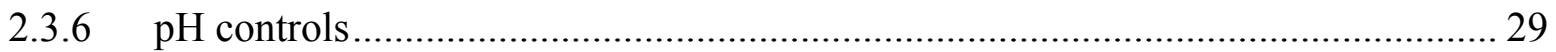

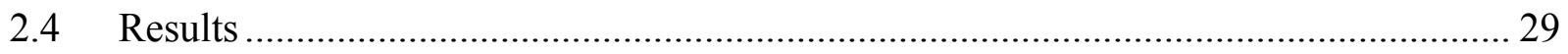

2.4.1 Antibiotic susceptibility of mixed species biofilms grown in $0.3 \mathrm{~g} / \mathrm{L}$ TSB medium 29

2.4.2 Added carbon during antibiotic exposure on young biofilms grown in $0.3 \mathrm{~g} / \mathrm{L}$ TSB medium (containing $0.14 \mathrm{mM}$ glucose) ........................................................................... 29

2.4.3 Effect of additional carbon during antibiotic exposures on biofilms grown in low

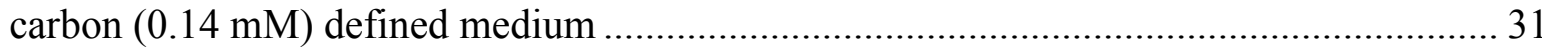

2.4.4 Biofilm metabolic response while switching carbon sources with and without antibiotic present ............................................................................................................ 33

2.4.5 Single species biofilm studies to determine the effect of streptomycin on $P$.

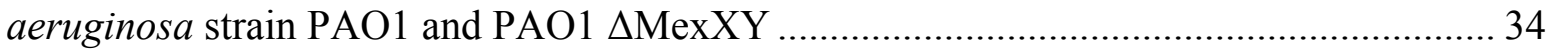

2.4.6 Adaptation and comparison to other Gram-negative strains.................................. 36

2.4.7 Minimum inhibitory concentration of streptomycin in various media types.......... 37

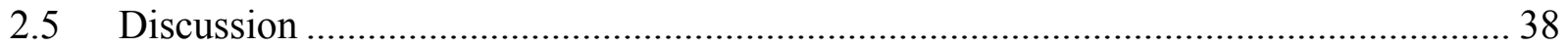

3 The influence of $\mathrm{pH}$ on whole-biofilm susceptibility to gentamicin and streptomycin as

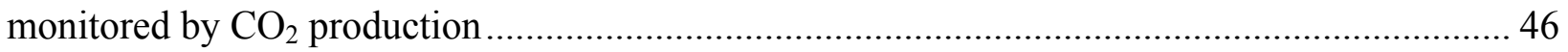

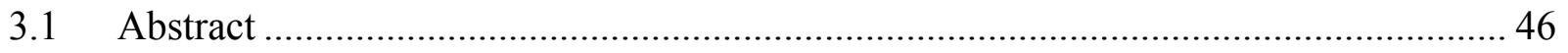

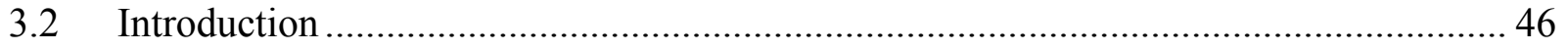

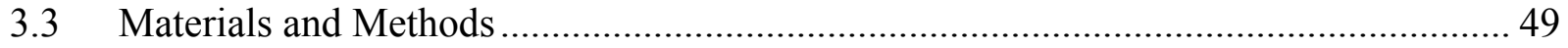

3.3.1 Bacterial strains and culture conditions ............................................................... 49

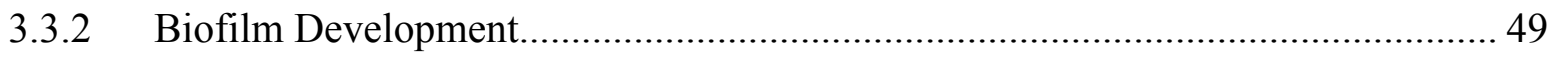

3.3.3 Measuring effluent $\mathrm{pH}$ following $\mathrm{pH}$ adjustments on growth medium fed to steady state biofilms 49

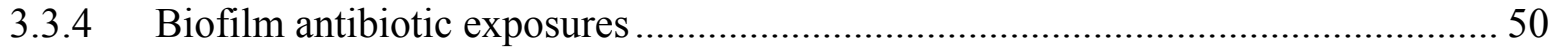

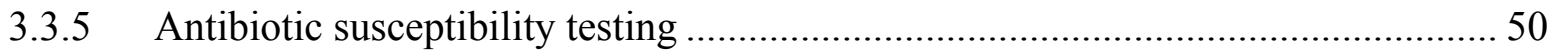




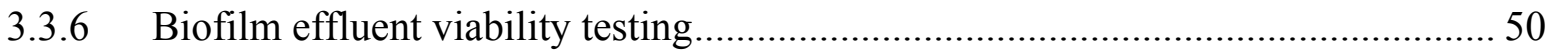

3.3.7 Flow cell experiments to study effect on biofilm structure …………………….... 51

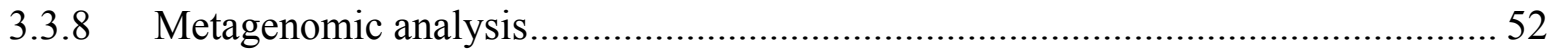

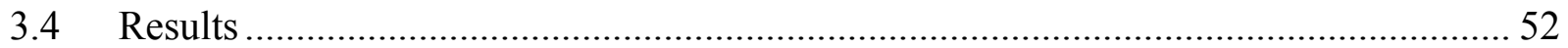

3.4.1 Biofilm adjustment of local environment in response to $\mathrm{pH}$ shifts .......................52

3.4.2 Antibiotic exposures on biofilms at various $\mathrm{pH}$............................................. 52

3.4.3 Biofilm effluent MIC values before and after exposures to high dose streptomycin

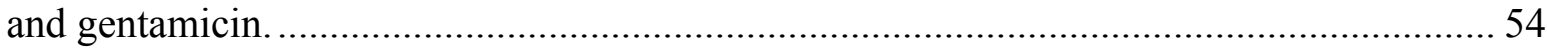

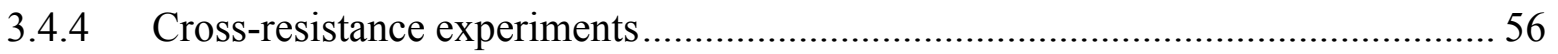

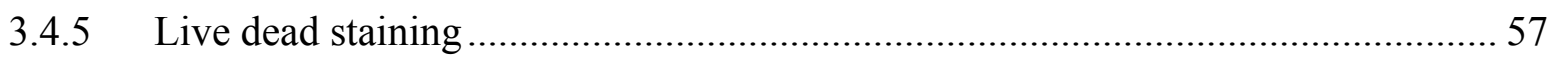

3.4.6 Metagenome analysis results ....................................................................... 59

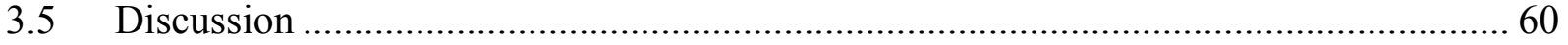

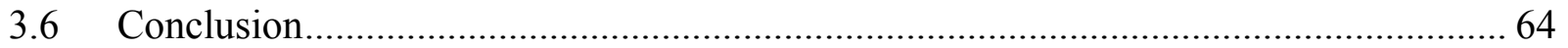

Multispecies biofilms: potential benefits for antibiotic resistance …………………................ 65

4 Multispecies biofilms: potential benefits for antibiotic resistance ........................................ 66

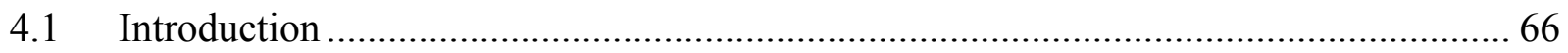

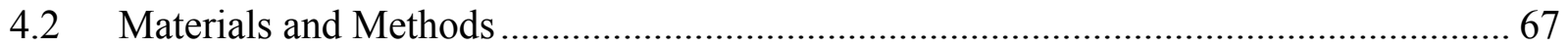

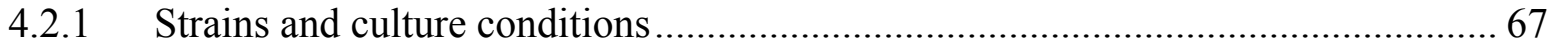

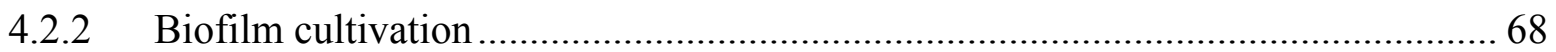

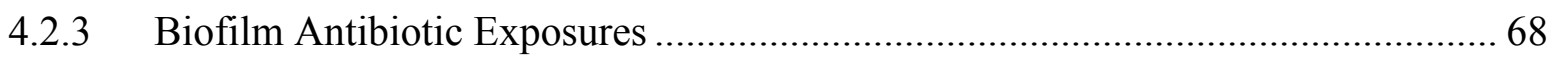

4.2.4 Whole-biofilm and biofilm effluent sample preparation for DNA extraction........ 68

4.2.5 Preparation of genomic DNA for PCR and genome sequencing ............................ 69

4.2.6 Preparation of plasmid DNA for PCR amplification............................................. 69

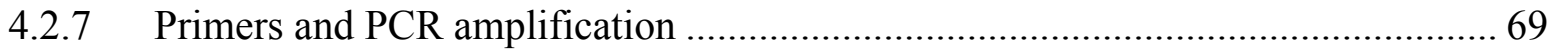

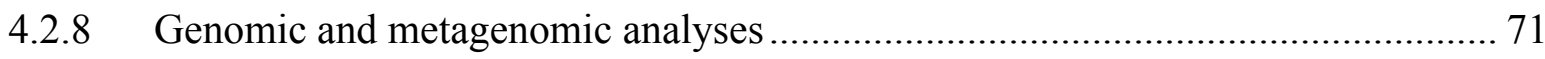

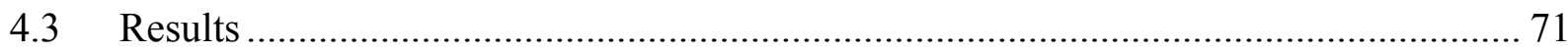

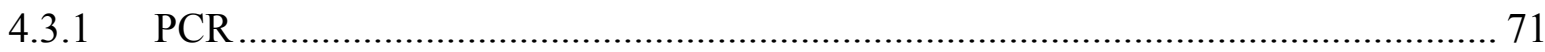

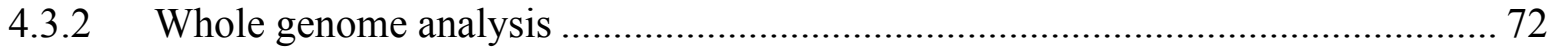

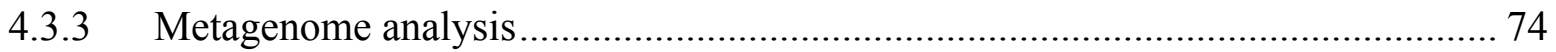

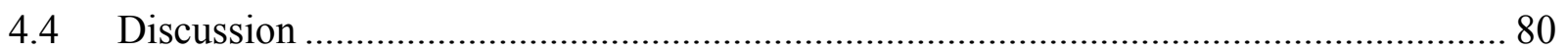

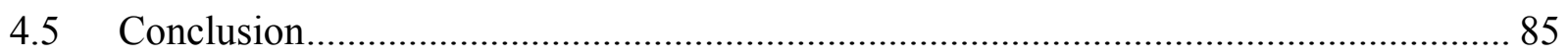

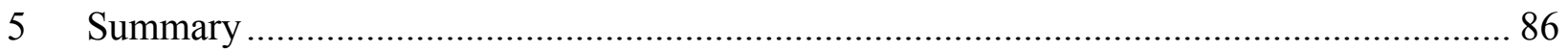

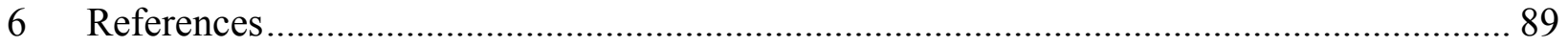




\section{List of Tables}

Table 3.1 Gentamicin $(\mathrm{Gm})$ and streptomycin (Str) MIC's of multispecies overnight cultures and steady state biofilm effluent before and after antibiotic exposures. Effluent collected from biofilms that did not recover from the antibiotic exposures did not produce viable cells, thus could not be tested for their planktonic MIC (in the chart labeled as "No recovery"). 55

Table 4.1. Primers used for PCR amplification of the $16 \mathrm{~S}$ region of DNA and for streptomycin modifying enzyme detection. 70

Table 4.2 Gel electrophoresis results combined with product sequencing results for PCR amplicons with the streptomycin modifying enzymes (SME) on the genomic DNA of the multispecies freezer stocks, multispecies bench cultures, the Gram-negative environmental isolates, and a negative control from a known species (E. coli EMG A20). The only PCR product that returned a sequence relating to a SME was the $\operatorname{aph}(3$ ") PCR product amplified from the $P$. putida isolate. *The section of DNA PCR amplified with the U341 and U378 primers identified the freezer cultures and bench cultures as $P$. aeruginosa. 72 Table 4.3. Subsystem feature counts obtained for the following genomes: PAO1 from GenBank, the multispecies bench culture. The rows in grey represent subsystems in which all of the gene counts are the same. 73

Table 4.4. The subsystem categories found under Virulence, Disease, and Defense following genome annotations of the PAO1 bench culture on the RAST server.

Table 4.5. The number of hits (unique sequence reads) from the subsystem categories of functional genes for each of the culture-derived genomic DNA sequences annotated using the mg-RAST server.

Table 4.6. Annotated aminoglycoside resistance related genes from metagenomic analysis. Each culture is present and numbered 1-7 as follows: 1) Planktonic Freezer stock in $3 \mathrm{~g} / \mathrm{L} \mathrm{TSB}, 2$ ) Planktonic bench culture in $3 \mathrm{~g} / \mathrm{L} \mathrm{TSB}, 3$ ) Biofilm grown in $0.3 \mathrm{~g} / \mathrm{L} \mathrm{TSB}$, 4) Pre-exposure biofilm effluent in $0.3 \mathrm{~g} / \mathrm{L} \mathrm{TSB}$, 5) Post-exposure biofilm effluent in $0.3 \mathrm{~g} / \mathrm{L} \mathrm{TSB}$, 6) Preexposure biofilm effluent in $1 \mathrm{mM}$ citrate medium, and 7) Post-exposure biofilm effluent in 1 $\mathrm{mM}$ citrate grown medium. All functional annotations were sourced from Genbank, and the genes were categorized according to function. The abundance signifies how many times that particular gene was found, the average \% identity represents how closely the annotated genes from the samples submitted match the Genbank gene sequences, and the number of hits represents the total unique sequence reads for the gene identified. *The EC numbers from the KEGG database were given for the genes that were annotated from multiple species or were from plasmids (Kanehisa and Goto, 2000). 


\section{List of Figures}

Figure 1.1. Monitoring of biofilm metabolism through the capture of $\mathrm{CO}_{2}$ production in realtime. (A) The CEMS system was set-up with media being fed into a gas-permeable inner silicone tube in the CEMS using a peristaltic pump. $\mathrm{CO}_{2}$ released by biofilms in the inner tube cross the silicon wall into the annular space confined by a gas-impermeable outer tygon tube, from where it is collected and transferred by $\mathrm{CO}_{2}$-free sweeper gas to a $\mathrm{CO}_{2}$ analyzed. (B) A cross-section of the CEMS showing flow of growth medium (liquid) and gas, separated by a gas-permeable silicone. 16

Figure 2.1. The $\mathrm{CO}_{2}$ production $(\mu \mathrm{mol} / \mathrm{h}$ ) of multispecies biofilms grown on $0.3 \mathrm{~g} / \mathrm{L}$ TSB medium exposed to $4000 \mathrm{mg} / \mathrm{L}$ streptomycin with and without the addition of carbon to the antibiotic medium. Column A delineates late exponential phase (A1) and early steady state biofilms (A2-A4) exposed to streptomycin. The biofilms were exposed to streptomycin in either $0.3 \mathrm{~g} / \mathrm{L}$ TSB medium $(\mathrm{A} 1-\mathrm{A} 3$ ) or $0.3 \mathrm{~g} / \mathrm{L}$ TSB medium with the addition of $1 \mathrm{mM}$ citrate to the $0.3 \mathrm{~g} / \mathrm{L}$ TSB antibiotic medium (A4). Column B delineates the metabolic response of biofilms that recovered following the high dose streptomycin exposures from the addition of either $3 \mathrm{~g} / \mathrm{L}$ TSB (B1), $1 \mathrm{mM}$ glucose (B2), or $2 \mathrm{mM}$ pyruvate (B3) to the antibiotic medium................... 31 Figure 2.2. The $\mathrm{CO}_{2}$ production $(\mu \mathrm{mol} / \mathrm{h}$ ) of multispecies biofilms grown in $0.14 \mathrm{mM}$ citrate defined medium and exposed to $4000 \mathrm{mg} / \mathrm{L}$ streptomycin for 4-h intervals (A) $0.14 \mathrm{mM}$ citrate medium (B) $0.14 \mathrm{mM}$ citrate growth medium with the addition of $1 \mathrm{mM}$ citrate during the first and second exposure, and a third antibiotic exposure in $0.14 \mathrm{mM}$ citrate medium.................... 32 Figure 2.3. The $\mathrm{CO}_{2}$ production $(\mu \mathrm{mol} / \mathrm{h}$ ) of multispecies biofilms grown in defined medium: (A) $1 \mathrm{mM}$ glucose as the carbon source exposed two times to $1 \mathrm{mM} 4000 \mathrm{mg} / \mathrm{L}$ streptomycin in $1 \mathrm{mM}$ citrate, (B) $1 \mathrm{mM}$ citrate exposed two times to $4000 \mathrm{mg} / \mathrm{L}$ streptomycin in $1 \mathrm{mM}$ glucose (C) $1 \mathrm{mM}$ citrate grown biofilms exposed to $1 \mathrm{mM}$ antibiotic free glucose (D) $1 \mathrm{mM}$ glucose grown biofilms exposed to $1 \mathrm{mM}$ antibiotic free citrate.

Figure 2.4. $\mathrm{CO}_{2}$ production $(\mu \mathrm{mol} / \mathrm{h}$ ) of biofilms of $P$. aeruginosa PAO1 (column $\mathrm{A}$ ) and PAO1 $\triangle \mathrm{MexXY}$ (column B) grown in $0.3 \mathrm{~g} / \mathrm{L}$ TSB medium. The biofilms were grown for less than 48 $\mathrm{h}$ and had reached steady state $\mathrm{CO}_{2}$ output before they were exposed to $4000 \mathrm{mg} / \mathrm{L}$ streptomycin (str) for $4 \mathrm{~h}$. Figures (A1) and (B1) biofilms were exposed to str in $0.3 \mathrm{~g} / \mathrm{L} \mathrm{TSB}$ medium. Figures (A2) and (B2) were exposed to str in $0.3 \mathrm{~g} / \mathrm{L}$ TSB medium in $0.3 \mathrm{~g} / \mathrm{L}$ TSB with the addition of $0.86 \mathrm{mM}$ glucose (glu).

Figure 2.5. The $\mathrm{CO}_{2}$ production $(\mu \mathrm{mol} / \mathrm{h}$ ) of biofilms of $P$. aeruginosa PAO1 (column A) and PAO1 $\triangle$ MexXY (column) grown in $0.3 \mathrm{~g} / \mathrm{L}$ TSB medium. The biofilms inoculated from bench cultures were grown for less than $48 \mathrm{~h}$ and had reached steady state $\mathrm{CO}_{2}$ output before they were exposed to $4000 \mathrm{mg} / \mathrm{L}$ streptomycin (str) for $4 \mathrm{~h}$. Figures (A1) and (B1) biofilms were exposed to str in $0.3 \mathrm{~g} / \mathrm{L}$ TSB medium. Figures (A2) and (B2) were exposed to str in $0.3 \mathrm{~g} / \mathrm{L}$ TSB medium in $0.3 \mathrm{~g} / \mathrm{L} \mathrm{TSB}$ with the addition of $0.86 \mathrm{mM}$ glucose (glu).

Figure 2.6. The $\mathrm{CO}_{2}$ production $(\mu \mathrm{mol} / \mathrm{h}$ ) of bacterial isolates exposed to streptomycin in $0.3 \mathrm{~g} / \mathrm{L}$ TSB medium for 4-h intervals. (A) multispecies bench culture grown on $0.3 \mathrm{~g} / \mathrm{L}$ TSB medium and exposed to $4000 \mathrm{mg} / \mathrm{L}$ streptomycin. The effluent cell numbers decreased from $2.0 \times 10^{7}$ $\mathrm{CFU} / \mathrm{ml}$ at steady state to $9.0 \times 10^{2} \mathrm{CFU} / \mathrm{ml}$ at the end of the first $4 \mathrm{~h}$ exposure. In contrast, the second exposure did not result in a similar decrease of effluent cell numbers; which were $2.4 \times 10^{5}$ 
$\mathrm{CFU} / \mathrm{ml}$ at the end of the $4 \mathrm{~h}$ exposure (B) P. aeruginosa on $0.3 \mathrm{~g} / \mathrm{L}$ TSB medium and exposed to either 4000, 8000, or 12,000 mg/L streptomycin (C) an Enterobacter species biofilm (D) Enterobacter asburiae biofilm, (E) a Pseudomonas putida biofilm, and (F) multispecies biofilm grown for 5 days prior to the streptomycin exposure. 38 Figure 3.1 Multispecies biofilms were grown in $0.3 \mathrm{~g} / \mathrm{L}$ TSB medium until a steady state $\mathrm{CO}_{2}$ output was reached prior to being exposed for $4 \mathrm{~h}$ to $4000 \mathrm{mg} / \mathrm{L}$ of either gentamicin (Column A:

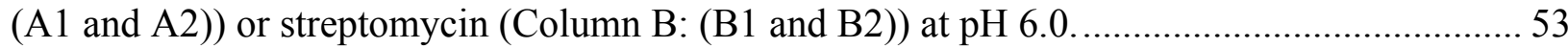
Figure 3.2. Multispecies biofilms were grown in $0.3 \mathrm{~g} / \mathrm{L}$ TSB medium until a steady state $\mathrm{CO}_{2}$ output was reached prior to being exposed for $4 \mathrm{~h}$ to $4000 \mathrm{mg} / \mathrm{L}$ of either gentamicin ((Column A: (A1 and A2)) or streptomycin (Column B: (B1 and B2)) at pH 7.0......

Figure 3.3. Multispecies biofilms were grown in $0.3 \mathrm{~g} / \mathrm{L}$ TSB medium until a steady state $\mathrm{CO}_{2}$ output was reached prior to being exposed for $4 \mathrm{~h}$ to $4000 \mathrm{mg} / \mathrm{L}$ of either gentamicin ((Column A: (A1 and A2)) or streptomycin (Column B: (B1 and B2))at pH 7.4. 55 Figure 3.4. Multispecies biofilms grown in $0.3 \mathrm{~g} / \mathrm{L}$ TSB medium and exposed to (A) $4000 \mathrm{mg} / \mathrm{L}$ streptomycin at pH 7.03 times, followed by two subsequent $4000 \mathrm{mg} / \mathrm{L}$ gentamicin exposures at pH 7.0 (B) $4000 \mathrm{mg} / \mathrm{L}$ gentamicin at pH 7.4, followed by one $4000 \mathrm{mg} / \mathrm{L}$ streptomycin exposure

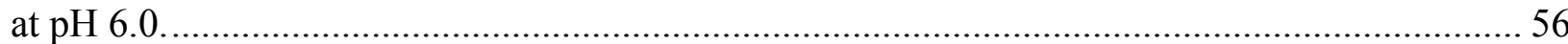
Figure 3.5. Multispecies biofilms that were grown in flow cells in $0.3 \mathrm{~g} / \mathrm{L}$ TSB were stained with Syto9 and Propidium Iodide for visualization of live versus dead biomass composition. (A) The pH 6.0 control showed an average of less than $0.5 \%$ biomass compared to the total biomass and the gentamicin exposures at $\mathrm{pH} 6.0 \mathrm{had}$ an average of $55 \%$ dead biomass in the biofilm at $168 \mathrm{~h}$ after exposure. (B) The $\mathrm{pH} 7.4$ control showed an average of less than $0.5 \%$ biomass compared to the total biomass and the antibiotic treated biofilms had $6 \%$ dead at $168 \mathrm{~h}$ after exposure..... 57 Figure 3.6. Single CLSM micrographs representative of the multispecies biofilms stained with Syto 9 and Propidium Iodide. Biofilms $168 \mathrm{~h}$ after 4-h $4000 \mathrm{mg} / \mathrm{L}$ gentamicin exposure at $\mathrm{pH} 7.4$ in $0.3 \mathrm{~g} / \mathrm{L}$ TSB medium (A and $\mathrm{B}$ ) and $168 \mathrm{~h}$ after a 4-h antibiotic-free $\mathrm{pH} 7.4$ exposure in 0.3 g/L TSB medium (C and D).................................................................................. 58 Figure 3.7. Single CLSM micrographs representative of the multispecies biofilms stained with Syto 9 and Propidium Iodide. Biofilms $168 \mathrm{~h}$ after 4-h $4000 \mathrm{mg} / \mathrm{L}$ gentamicin exposure at $\mathrm{pH} 6.0$ in $0.3 \mathrm{~g} / \mathrm{L}$ TSB medium (A and $\mathrm{B}$ ) and $168 \mathrm{~h}$ after a 4-h antibiotic-free $\mathrm{pH} 6.0$ exposure in 0.3 $\mathrm{g} / \mathrm{L}$ TSB medium (C and D).....

Figure 4.1. The percent genus distribution of bacterial 16S DNA present in the multispecies (A) freezer stock, (B) biofilm, (C) pre-streptomycin exposure effluent and, (D) post-streptomycin exposure effluent. The figure demonstrates how the multispecies community shifted from planktonic state to biofilm and following a streptomycin exposure in $0.3 \mathrm{~g} / \mathrm{L}$ TSB medium. The dark-grey boxes demonstrate the shift of Stenotrophomonas in the community. 76 Figure 4.2.The percent genus distribution of bacterial 16S DNA present in the multispecies (A) bench cultures grown in $0.3 \mathrm{~g} / \mathrm{L} \mathrm{TSB},(\mathrm{B})$ effluent collected from pre-streptomycin exposure in 1 $\mathrm{mM}$ citrate medium and, $(\mathrm{C})$ effluent collected from pre-streptomycin exposure in $1 \mathrm{mM}$ citrate medium. The black boxes highlight the fact that each of these cultures was largely dominated by 


\section{List of Abbreviations:}

BLAST

CEMS

CF

$\mathrm{CFU} / \mathrm{mL}$

CLSM

DNA

eDNA

EPS

gfp

HIV

MIC

MBC

MBEC

OD600

PCR

mg-RAST

RNA

TSA

TSB

VDD
Basic Local Alignment Search Tool

Carbon dioxide evolution measurements system

Cystic fibrosis

Colony forming units per millilitre

Confocal laser scanning microscopy

Deoxyribonucleic acid

Extracellular DNA

Extracellular polymeric substance

Green fluorescent protein

Human immunodeficiency virus

Minimum inhibitory concentration

Minimum bactericidal concentration

Minimum biofilm eradication concentration

Optical density at $600 \mathrm{~nm}$

Polymerase chain reaction

Metagenomic-Rapid Annotation using Subsystem Technology

Ribonucleic acid

Tryptic soy agar

Tryptic soy broth

Virulence, Disease and Defence 
Chapter 1

Introduction 


\section{Introduction}

\subsection{Biofilm Nature and Pathogenicity}

In nature, bacteria often exist in heterogeneous communities called biofilms. Bacteria can establish themselves in sessile communities or biofilms by excreting a self-produced extracellular polymeric substance (EPS) that embeds the cells and allows for attachment to biotic and abiotic surfaces (Donlan, 2002). Evidence of biofilm growth has been dated back approximately 3.3 billion years ago; allowing for stability of bacterial living conditions in harsh and changing environments (Hall-Stoodley et al., 2004). In both nature and disease, biofilms have proven extremely beneficial to the bacteria within. For example a single cell inside a human host remains open and exposed to the immune system to which it is often easily identified and destroyed whereas in biofilm, antigens present on bacterial cells can remain hidden from the host's immune system (Wolcott et al., 2008).

Microbial infections were the main cause of death in humans until the advent of antibiotics. Although microbial infections may no longer be the leading cause of death in developed countries, they remain an important issue. Hospital-acquired infections cost the US alone billions of dollars annually, with more than 2 million patients affected each year. Sixty to seventy percent of these infections are caused by implanted medical devices where biofilms can form (Bryers et al., 2008). With the rise in antibiotic resistance and the difficulty in treating biofilm infections in particular, there remains a pressing need to study biofilm behaviour. Bacterial biofilms are well known to the medical community as resilient infectious agents that are difficult to eradicate. More than $65 \%$ of bacterial infections in humans are caused by biofilm forming bacteria (Nithya et al, 2010). Individuals who have a weakened immune system are more likely to acquire biofilm infections, such as patients with human immunodeficiency virus (HIV), cystic fibrosis (CF), or those with severe burns (Costerton et al., 1999). A relatively high percentage of nosocomial infections $(10 \%$ to $20 \%)$ are caused by the formation of biofilms on a patient's medical device such as a contact lens, artificial joint or synthetic valve (Nithya et al., 2010 and Costerton et al., 1999). Important human infections that involve biofilms include native valve endocarditis, otitis media, chronic bacterial prostatitis, CF, and periodontitis. After having formed a biofilm, bacteria can persist for a long time in a patient or on their attached medical device(s) leading to chronic infections (Costerton et al., 1999). An individual who has a chronic infection due to 
bacterial biofilm development would be asymptomatic while hosting a community of bacteria. However, dangerous infections arise sporadically as a result of bacterial dispersal from the biofilm into the individual's systemic circulation (Marks et al., 2013). The intermittent systemic infections increase the odds of severe health complications leading to a greater risk of fatality (Hall-Stoodley and Stoodley, 2009). A chronic infection allows not only for bacterial adaptation to the host's environment, but for manipulation of the host's immune system in order to further benefit the biofilm and supply it with nutrients needed to persist and thrive (Hall-Stoodley and Stoodley, 2009; Wolcott et al., 2008). The inflammatory response is meant to combat infection, though bacteria such as Pseudomonas aeruginosa can produce signalling molecules that influence the functioning of mammalian host cells for their benefit (Jahoor et al., 2008). Whether bacterial biofilms are manipulating their host's environment or it is their own physiology that leads to chronic infections, they are difficult to eliminate due to the variety of defence mechanisms. Studies have shown that when biofilm forming bacteria aggregate within a biofilm they can be 10 to a 1000 times more resistant to antibiotics compared to bacteria in planktonic state (Nithya et al., 2010; Mah and O’Toole, 2001). Better understanding of resistance should lead to new methods of combating the diseases and life threatening infections caused by biofilms.

Stewart (2002) outlines five mechanisms in which biofilms can circumvent the detrimental effects of antibiotics: (1) poor antibiotic penetration, (2) nutrient limitation, (3) slow growth, (4) adaptive stress responses and, (5) the formation of persister cells. Mechanisms (2) through (5) also apply to bacteria that live in the planktonic state. Other resistance mechanisms that bacteria in both planktonic and biofilms can develop include, but are not limited to, target mutations, low cell permeability, efflux pumps, and antibiotic-modifying enzymes (Stewart, 2002).

Poor antibiotic penetration of the biofilm can occur from binding of antibiotics to substances present in the EPS. For instance a common component of the extracellular matrix of $P$. aeruginosa is the negatively-charged alginate that can bind to positively charged aminoglycoside antibiotics. Also, coagulase-negative Staphylococci can excrete slime that negatively affects glycopeptide antibiotic activity (Fux et al., 2005; Tseng et al., 2013; Davenport et al., 2014). Moreover, bacterial cells embedded in the EPS have added protection from retention of secreted enzymes that remain in the EPS and act to destroy antibiotics and antimicrobials (Gilbert et al., 
2002). Secretion of catalase can impede penetration of hydrogen peroxide and secreted betalactamases can disable beta-lactam antibiotics (Fux et al., 2005). Despite mechanisms that reduce the penetration of antibiotics through the EPS or destroy antimicrobials prior to reaching embedded cells, it was found that these mechanisms alone cannot account for resistance or tolerance to very high antibiotic concentrations. Stewart (2002) argues that a slight reduction in antibiotic mobility in biofilms cannot account for the extremely high resistance to killing with high-dose antibiotic exposures, because high antibiotic concentrations retain the ability to penetrate the biofilm.

The second antibiotic protective mechanism described by Stewart (2002) is that of nutrient limitation. Within the biofilm there are areas where nutrients are limited, such as in the depths of the biofilm due to continuous nutrient consumption in the outer layers. According to Fux et al. (2005), the bacteria in the depths of the biofilm are in a similar state to bacteria that have reached stationary phase in planktonic solutions. Close to the attachment surface there are limited nutrients and high cell densities creating competition for nutrients and leaving certain members of the community starved. The majority of antibiotics target physiological processes linked to growth and replication (Cotsonas and $\mathrm{Wu}, 2009$ ), thus lack of growth and replication will protect cells by leaving antibiotics without a target. It has been demonstrated that non-growing cells regain antibiotic susceptibility once diluted into a fresh medium and start growing again (Fux et al., 2005).

Slow growth and adaptation to stress are the third and fourth mechanisms that incur protection for biofilm-forming bacterial species (Stewart, 2002). Slow growth, similarly to starved/nongrowing cells, can aid bacteria in resisting most antibiotics since antibiotics normally target some form of macromolecular synthesis which occurs during exponential growth (Eng et al., 1991; Gilbert and Brown, 1988; Stewart, 2002). Thus, mature biofilm cells, being similar to bacteria in the stationary phase of planktonic growth, may not be producing many new molecules. The fourth protective mechanism that biofilms can use against antibiotics is adaption to stress. Stewart (2002) proposes the idea that bacteria in biofilms may have more time to express traits that prevent antibiotic susceptibility due to the retardation of antibiotics within in the biofilm. One example that demonstrates this point is the ability of $P$. aeruginosa biofilms to induce the $k a t B$ gene, a catalase gene, upon treatment with $50 \mathrm{mM}$ hydrogen peroxide. A planktonic culture 
of the same strain of $P$. aeruginosa was unable to express catalase upon peroxide treatment (Elkins et al., 1999). In the planktonic state, the antimicrobial overwhelms the bacteria before the stress response is activated (Stewart, 2002).

The final and most important mechanism that biofilms have to protect themselves against antimicrobials is the formation of persister cells (Lewis, 2010; Conlon et al., 2013; Keren et al., 2004a). Persister cell formation is suggested as a possible mechanism that biofilms use to survive extremely high concentrations of antibiotics, as well as higher than normal concentrations of heavy metals and antimicrobials (Stewart, 2002 and Harrison et al., 2005). Fux et al. (2005) describe persister cell formation as passive protection against antibiotics and host defences due to metabolic inactivity. Persister cells are formed through redundant mechanisms. Some examples include: toxin-antitoxin systems, genes affecting global regulators, enzymes involved in amino acid synthesis and metabolism, DNA-repair (CMPG13400), nutrient uptake or phospholipid metabolism, and quorum sensing molecules (Lewis, 2010; Groote et al., 2009; Möker et al., 2010). Biofilms and stationary phase planktonic cells generally have a higher persister cell population than actively-growing planktonic cells (Keren et al., 2004a). The presence of persister cells offers an explanation for broad resistance to antibiotics since these cells have entered a highly protected and spore-like state. Therefore, persisters would be unaffected for a time by antibiotics in their environment. However, this mechanism of antibiotic tolerance cannot account for all resistance. The opportunistic pathogen $P$. aeruginosa has been shown to produce low levels of persister cells (likely due to fitness associated trade-offs) and other mechanisms of resistance remain important for this pathogen's survival (Stepanyan et al., 2015).

Other researchers have similar suggestions as to how biofilms permit antibiotic resistance to antimicrobials. Donlan and Costerton (2002) attributed increased resistance to antimicrobials in biofilms to reduced penetration through the EPS, differential growth rate of bacteria within biofilms, and other physiological changes that result when bacteria grow in a biofilm. Whether the mechanisms of resistance are physiological, genetic or environmental, it seems that complete eradication of biofilms will be a difficult task due to many overlapping mechanisms of resistance of bacteria living within a biofilm. In addition, many of the antibiotic-resistance studies have been performed on single-species biofilms. Multispecies biofilms would certainly add 
complexity to the issue of antimicrobial resistance as each species would make different contributions genetically and physiologically to the biofilm (Giaouris et al., 2013; Simões et al., 2009; Hoa et al., 2015).

The motivation to study biofilm behaviour in response to antibiotics developed from the interest to learn more in relation to the proposed reasons of biofilm resistance. Biofilm survival following high dose antibiotic exposures has often been attributed to the large population of slow growing or dormant cells within the biofilm. Other mechanisms of multidrug resistance result from active resistance mechanisms. It is shown that biofilms are dynamic, responding rapidly to environmental changes. Biofilms can survive very high doses of antibiotics through activity as oppose to inactivity.

\subsection{Motivation for the Study}

Biofilm survival following high dose antibiotic exposures has often been attributed to the large population of slow growing or dormant cells within the biofilm. However, other mechanisms of multidrug resistance result from active mechanisms. Both active and passive resistance mechanisms can be reflected in overall metabolic output. Therefore, in the interest of understanding the dynamic nature of biofilms and their response to antibiotic exposures the following study chose to monitor whole-biofilm $\mathrm{CO}_{2}$-production of multispecies biofilms during high-dose aminoglycoside exposures. It was hypothesized that the multispecies biofilms (containing the opportunistic pathogens $P$. aeruginosa and $S$. maltophilia) would be susceptible to high-dose antibiotic exposures. Furthermore, it was hypothesized that changing environmental parameters, such as added nutrients and more acidic pHs during aminoglycoside exposures, would decrease aminoglycoside efficacy and biofilm susceptibility.

\subsection{Antibiotic Resistance}

\subsubsection{Opportunistic Pathogens}

In the study of biofilm-related infections and diseases, especially in the medical community, the term opportunistic pathogen is commonly used. Brown et al. 2012 have defined opportunistic pathogens as "non-obligate and/or non-specialist parasites of a focal host." Opportunistic pathogens can be found living on human hosts or in environmental settings (for example 
Staphyloccocus aureus and P. aeruginosa, respectively) (Brown et al., 2012). Opportunistic pathogens are normally harmless, but can become pathogenic and cause great harm to humans that have fallen ill or become injured. Opportunistic pathogens can exhibit high degrees of antimicrobial resistance, and often can form biofilm infections.

The bacterium Stenotrophomonas maltophilia is an example of an opportunistic pathogen. It has been found in a variety of different environments such as Antarctica, rivers, raw milk, sewage, in soils, vegetation, etc. (Spencer, 1995; Denton and Kerr; 1998). Recently the biofilm-forming $S$. maltophilia has been linked to many diseases such as pneumonia, osteomyelitis, endocarditis, meningitis, CF, and many more (Brooke, 2012; Spencer, 1995). Other biofilm-forming organisms, such as $P$. aeruginosa, are also found in many different environmental settings. Although rather harmless to healthy individuals, it can be pathogenic for an immunocompromised host (Wolfgang et al. 2003).

P. aeruginosa is one of the most studied biofilm-forming bacteria (Musk, 2005). Its mechanisms of pathogenicity are well described allowing for a good understanding of the complexity of antibiotic resistance in biofilms. $P$. aeruginosa is a prevalent opportunistic pathogen and is a common cause of nosocomial infections that readily infects immunosuppressed patients including ones with HIV or CF and patients with severe burns or with implanted medical devices (Hentzer, 2002; Costeron et al. 1999; Nithya et al., 2010).

Bacterial colonization of a CF lung is a good example of a site of injury in an individual that will lead to the opportunistic infections in a host. In CF, initial lung infections are often caused by $P$. aeruginosa, Staphylococcus aureus, and Haemophilus influenza (Starner et al., 2005). When the CF lung is colonized with $P$. aeruginosa, Burkholderia cepacia infections are more likely to become life-threatening. Other pathogens commonly associated with $\mathrm{CF}$ include: Nontuberculous mycobacteria, Achromobacter xylosoxidans, and S. maltophilia (Gabriel et al., 2004). Studies have shown that by 3 years of age $98 \%$ of patients with CF have P. aeruginosa infections (Golvan et al., 2007). The mucoid phenotype of $P$. aeruginosa develops over time and enables successful colonization of the lungs. This increases its ability to evade the host's immune system, exacerbates the immune response and increases resistance to antibiotics (Koch and Hoiby, 1993; Pedersen et al., 1992). The maturity level of the biofilm has also been a factor in 
the susceptibility of the organism to the host's immune system's defences. $t$ appears that older, more established, biofilms are more difficult for the host to eliminate (Hentzer et al., 2001). The underlying reasons for increased survival of aged biofilms may include increased thickness, population density, changes in gene expression, and inactivation of lower layers of the biofilm (Stewart, 2002; Stewart, 1994). Antibiotics are traditionally used to aid with systemic and biofilm bacterial infections. Therefore genetic antibiotic resistance mechanisms, whether intrinsic or acquired, are of interest in addition to biofilm protective mechanisms.

\subsubsection{Antimicrobial Resistance Mechanisms in Pseudomonas aeruginosa}

Much time and energy has been spent trying to uncover all aspects of antibiotic resistance in bacteria, because the arrival antibiotics radically changed the likelihood of survival following a bacterial infection. Antibiotics are indispensible and have likely saved more lives than any other drug (Aminov, 2010; Sulakvelidze, 2005; Dryden et al., 2009). Recently, the view on the effectiveness of antibiotics has become bleak due to the rise in antibiotic resistance and the inability to treat patients infected with resistant organisms.

For one of the most studied biofilm-forming bacteria and a prevalent opportunistic pathogen, $P$. aeruginosa, many antibiotic resistance mechanisms have been uncovered. Moore and Flaws (2011) placed resistance mechanisms for P. aeruginosa into one of three categories: 1) genetically encoded resistance; 2) mutations; or 3) acquired resistance through genetic transfer of plasmids carrying antimicrobial resistant genes.

Intrinsic antibiotic resistance mechanisms in P. aeruginosa include: multidrug efflux pumps, reduction in outer-membrane permeability and antimicrobial degrading enzymes (De Kievit et al. 2001; Moore and Flaws, 2011). Naturally-occurring efflux pumps that are used by bacteria to extrude toxic substances from within their cells can aid in preventing the accumulation of a variety of antibiotics (Lomovskaya et al., 2001). Multidrug efflux pumps require energy from the proton motive force, except efflux pumps from the ABC family that make use of ATP, to actively remove antibiotics (Lister et al, 2009). In P. aeruginosa, efflux pumps can extrude various classes of antibiotics, including: beta-lactams, aminoglycosides, macrolides, and fluoroquinolones (Moore and Flaws, 2011). In addition to efflux pump function, intrinsic

antibiotic resistance can occur through changes in outer membrane permeability. Beta-lactam 
and quinolone antibiotics utilize outer membrane porins to enter the cell. Decreased outer membrane permeability thus limits access of these antibiotics to their targets within the cell. Aminoglycoside antibiotics do not use porin channels to cross the outermembrane (Moore and Flaws, 2011). Instead, they attach to lipopolysaccharides (LPS) on P. aeruginosa's outer membrane, increasing the membrane permeability and allowing the aminoglycosides to reach the cytoplasm. The outermembrane protein oprH has been linked to colistin and aminoglycoside resistance by preventing LPS-antibiotic binding (Lambert, 2002). For the third intrinsic mechanism, antimicrobial degrading enzymes, only one known chromosomally-encoded gene exists that $P$. aeruginosa uses for antibiotic resistance. This is $a m p C$, which provides resistance to beta-lactam antibiotics through antibiotic degradation (Lister et al., 2009).

Moore and Flaws' (2011) second category of antimicrobial resistance mechanisms are those acquired through genetic mutation. In particular, fluoroquinolone antibiotic resistance does not occur through acquisition of genes, but from genetic mutations that change the antimicrobial targets. Fluoroquinolone antibiotics disrupt DNA replication by targeting the enzyme DNA gyrase, which consists of two subunits (GyrA and GyrB). DNA gyrase is a type II DNA Topoisomerase involved in DNA replication (Kureishi et al., 1994; Hooper, 2001). If mutations arise in the $g y r A$ or, less commonly, the $g y r B$ gene, antibiotic resistance can occur (Hooper, 2001). For example, $P$. aeruginosa can confer resistance to fluoroquinolones when the gyr $A$ gene is mutated, which alters the DNA gyrase GyrA subunit (Lister et al., 2009). In addition, mutations that alter the $30 \mathrm{~S}$ ribosome lead to streptomycin (an aminoglycoside) resistance. Streptomycin is the only aminoglycoside thus far to show loss of function due to ribosomal mutations (Moore and Flaws, 2011). Furthermore, beta-lactam antibiotic resistance can arise due to genetic mutations that alter the form of penicillin-binding proteins, which are responsible for cell wall synthesis and are beta-lactam antibiotic targets (Georgopapadakou, 1993; Moore and Flaws, 2011).

Acquisition of bacterial plasmids containing antibiotic resistance genes is the third category of antimicrobial resistance mechanisms described by Moore and Flaws (2011). There are three known classes of aminoglycoside modifying enzymes that $P$. aeruginosa obtains from plasmids. These are O-phosphotransferase (APH), O-Nucleotidyl transferase (ANT), and NAcetyltransferase (ACC). The latter two enzymes require ATP as a substrate in their reactions 
(Mingeot-Leclercq, 1999; Moore and Flaws, 2011). Beta-lactamases are a group of beta-lactam inhibiting enzymes that can be imported through plasmids. Plasmid-mediated fluoroquinolone resistance has never been demonstrated (Lister et al., 2009).

P. aeruginosa does have many ways to develop resistance against each class of antibiotics. However, there are still several classes of antibiotics that can be used effectively against pseudomonal infections. Patients infected with $P$. aeruginosa can be treated with aminoglycosides (tobramycin and gentamicin), variants of beta-lactams (ceftazidime and impenem), polymyxins, and fluoroquinolones (ciprofloxacin) (Geller, 2009; Su et al., 2010). The beneficial effects of each antibiotic are dependent upon the growth stage of the bacterial population. For example, ciprofloxacin is a fluoroquinolone antibiotic and is most effective on slow growing cells (Keren et al., 2004b). In contrast, tobramycin and gentamicin are aminoglycoside antibiotics that inhibit protein synthesis and are most effective against bacterial cells in the exponential phase of growth (Keren et al., 2004b).

The pathogenicity and virulence of $P$. aeruginosa extends beyond antibiotic resistance mechanisms. P. aeruginosa has virulence factors capable of altering a host's immune system and local environment. Though, the methods that contribute to the efficacy of $P$. aeruginosa as a pathogen will not be discussed. Biofilm infections are often a result of multiple species coinhabiting a wound. Each community may carry a variety of resistance genes and respond optimally to different antibiotic treatments. Therefore, it is as important to compile data on the responses of multispecies biofilms to antibiotic treatments as well.

\subsubsection{Antipseudomonal agents and antibiotics effective against Gram-negative bacteria}

Although $P$. aeruginosa has not always been considered an important pathogen, it is now one of the most common pathogens implicated in a wide range of hospital acquired infections (Giamarellou, 2002). Any mucosa membrane in the body or the skin of patients treated with antibiotics can be colonized with $P$. aeruginosa, but it is not a normal organism found on healthy patients (Iregbu and Eze, 2014; Giamarellou, 2002). Several classes of antibiotics have antipseudomonal activity, including; aminoglycosides, ticarcillin, ureidopenicillins, ceftazidime, cefepime, aztreonam, the carbapenems, and ciprofloxacin (Giamarellou and Antoniadou, 2001). The aminoglycosides gentamicin and tobramycin, and the fluoroquinolone ciprofloxacin are 
currently used as antipseudomonal agents in infections in CF patients (Rossolini and Mantengoli, 2003; Mogayzel et al., 2013; Geller, 2009; Su et al., 2010). Ciprofloxacin (a fluoroquinolone) is an antipseudomonal agent that has been show to be effective against non-growing cells, as biofilms have often been compared to stationary phase cultures (Chalkley and Koornhof, 1985; Eng et al., 1991; Brown et al., 1988). Aminoglycosides, though less effective against more slowly growing cultures, have been used over the years as important antipseudomonal agents, particularly in the treatment of CF lung infections (Poole, 2005; Eng et al., 1991).

\subsubsection{Streptomycin and Gentamicin Aminoglycoside Antibiotics}

Aminoglycoside are broad spectrum antibiotics that target the bacterial ribosome and prevent protein synthesis (Mingeot-Leclercq, 1999). There is a net negative charge inside bacterial cell cytoplasm that is believed to be the main driving force for the uptake of aminoglycoside that have a net positive charge at physiological pH (Bryan and Kwan, 1983; Damper, 1981). Aminoglycoside uptake is initiated by ionic binding to the cell membrane (Mandel et al., 1984) and in Gram-negative bacteria by disrupting the $\mathrm{Mg}^{2+}$ bridges between adjacent lipopolysaccharide (LPS) molecules in the outermembrane (Mingeot-Leclercq, 1999). There are two additional phases of aminoglycoside uptake following ionic binding; energy-dependent phase I and energy-dependent phase II. The second energy-dependent phase is enhanced by ribosomal binding (Bryan and Kwan, 1983).

Factors that affect aminoglycoside uptake include a surplus or scarcity of $\mathrm{Mg}^{2+}$ or $\mathrm{Ca}^{2+}$, changes in $\mathrm{pH}$, electrical potential and ATP production, (Mulcahy et al., 2008; Fraimow et al., 1991). Any process that causes changes in membrane permeability or energy production will likely affect aminoglycoside uptake and mode of action. For example, extracellular DNA affects membrane permeability because it binds to $\mathrm{Mg}^{2+}$ and $\mathrm{Ca}^{2+}$ ions that stabilize LPS molecules. This leads to membrane modifications and loss of ionic binding of streptomycin (Mulcahy et al., 2008). The uptake and lethality of streptomycin has been shown to require respiration (electron transport), since it has been antagonized by electron transport inhibitors, such as potassium cyanide, carbon monoxide, sodium azide etc (Hancock, 1981). 


\subsubsection{Applications for aminoglycosides in clinical and agricultural settings}

Aminoglycoside antibiotics have a wide range of applications. They are broad spectrum antibiotics, meaning they have activity against a range of Gram-positive and Gram-negative bacteria. They can also be used in clinical settings to combat infectious diseases or in agriculture to combat plant pathogens. The first aminoglycoside discovered, streptomycin, was isolated from a soil microorganism Streptomyces griseus in the 1940's (Emerson de Lima Procopio et al., 2012; Baltz, 2008; Kingston, 2004). Streptomycin is one of the primary drugs used to treat infections caused by Mycobacterium tuberculosis (Mariam et al., 2011). According to the FDA, in addition to the use of streptomycin in treating M. tuberculosis, the antibiotic can be used to treat infections caused by susceptible bacteria that are not responsive to less harmful antibiotic agents, including: Pasteurella pestis, Francisella tularensis, Brucella, Calymmatobacterium granulomatis, H. Ducreyi, H. Influenza, K. pneumonia pneumonia, E.coli, Proteus, A. aerogenes, K. pneumoniae, Enterococcus faecalis, Streptococcus viridans, Enterococcus faecalis, and Gram-negative bacillary bacteremia (http://www.drugs.com/pro/streptomycin.html, retrieved June 10, 2015). Streptomycin antibiotics have also been used heavily in agriculture since the 1950 's in order to perturb bacterial diseases on potatoes, tobacco and other vegetable seedlings, as well as being used for the treatment of blight (Stockwell and Duffy, 2012).

Following the discovery of streptomycin, which demonstrated success in treating bacterial infections, many other aminoglycosides were introduced as antibacterial agents and also proved to be successful. In particular, gentamicin was shown to be highly effective in treating Gramnegative bacterial infections (Mingeot-Leclercq et al., 1999). According to the FDA, gentamicin can be used to treat severe bacterial infections, including infections caused by: Pseudomonas aeruginosa, Proteus species (indole-positive and indole-negative), Escherichia coli, KlebsiellaEnterobacter-Serratia species, Citrobacter species and Staphylococcus species (coagulasepositive and coagulase-negative) (http:/www.drugs.com/pro/gentamicin-sulfate.html\#c4ed5f4f13df-44dc-87c5-6867e7edea7d, retrieved June 10, 2015). Gentamicin is an important antipseudomonal agent and is commonly used for bacterial infections in patients with $\mathrm{CF}$ (Rossolini and Mantengoli, 2003; Mogayzel et al., 2013). Gentamicin is also used to combat agricultural pests in Latin American countries, such as in Mexico, Chile, and Central American Countries, but not in the United States (US). Unlike the use of streptomycin for agriculture in the 
US, the use of gentamicin in agriculture is controversial considering its use in treating infectious diseases (Vidaver, 2002).

\subsubsection{Application of fluoroquinolones with special reference to ciprofloxacin}

Similarly to aminoglycoside antibiotics, fluoroquinolones are broad spectrum antibiotics. They target one of two enzymes (DNA gyrase or Topoisomerase IV) that are required for DNA synthesis (Hooper, 2001). Fluoroquinolone antibiotics are widely used in both veterinary and human medicine (Piddock, 1998). Ciprofloxacin, in particular, is one fluoroquinolone that is highly effective against Gram-negative bacteria. Some of the bacterial infections that ciprofloxacin is approved to treat include respiratory tract infections that commonly caused by Streptococcus pneumoniae, Haemophilus influenza or Moraxella catarrhalis (Hooper, 1998).

\subsubsection{Antibiotic Susceptibility Testing: Bacterial Biofilm and Planktonic Systems:}

In order to study antibiotic efficacy and the development of antibiotic resistance there need to be standards in place so that clinical laboratories can come to an agreement on when an organism is resistant to an antibiotic. In the United States, antibiotic breakpoints and antibiotic susceptibility testing standards are designated by the Clinical and Laboratory Standards Institute (CLSI). In Europe, they use the European Committee on Antimicrobial Susceptibility Testing (EUCAST). Antibiotic breakpoints are determined by minimum inhibitory concentrations (MICs), which are susceptibility tests performed on planktonic bacterial cultures. Over the years there has been increasing interest and need to determine biofilm antibiotic susceptibility, because the antibiotic MICs are not indicative of biofilm susceptibility to antibiotics. New systems are constantly developed to try and understand biofilm behaviour and antibiotic susceptibility. Each has their own advantages and limitations. Choosing a particular system for studying biofilms will all depend on the questions you want to answer and insights you would like to gain from the experimental system.

\subsubsection{Minimum Inhibitory Concentration (MIC) and Minimum Bacterial Eradication Concentration (MBEC)}

Antibiotic susceptibility testing is an essential part of clinical, agricultural, veterinary and environmental testing in order to determine antimicrobial resistance levels. Antibiotics are heavily relied on to treat all sorts of infections and diseases caused by bacteria. Thus, it is crucial 
to understand the level of antibiotic resistance in the populations being treated. Currently, MICs are the main method to test planktonic antibiotic susceptibility (Girard et al., 2010). The more that has been understood about biofilms the more apparent it became that the planktonic antibiotic susceptibility tests were not a good indication of biofilm survival to antibiotics. The development of new methods for biofilm antibiotic susceptibility emerged and the idea of a minimum biofilm eradication concentration was developed (MBEC). Both the MIC and MBEC methods have benefits and can be used in combination to increase understanding of the antibiotic susceptibility of a particular bacterial species under different physiological states.

According to Andrews (2002) the MIC of an antimicrobial is the minimum antibiotic concentration that will prevent visible growth of an overnight culture of bacteria. If the microorganism to be tested for antimicrobial susceptibility is an anaerobe, more time is allocated for the culture to grow since anaerobes grow at a slower rate. The minimum bactericidal concentration (MBC) is defined as the lowest concentration of an antimicrobial that will prevent growth of a bacterial species, even after the antimicrobial substance is removed from the media (Andrews, 2002). Minimum biofilm eradication concentration (MBEC) is defined as "the minimum concentration of antimicrobial that eradicates the biofilm" (Ceri et al., 2001). The MBEC assay is performed using a Calgary Biofilm Device (CBD). The CBD is a high throughput method that produces 96 biofilms on pegs in a plastic apparatus placed on a tilt-table allowing for flow conditions (Ceri et al. 2001). Many articles have determined the effectiveness of antibiotics in a biofilm by comparing the MBEC numbers to MIC of planktonic bacteria. However, this comparison is erroneous because, as stated above, the MIC only determines the amount of antibiotic that will inhibit growth of an overnight not completely eradicate it. MBC and MBEC can be better comparised since both methods determine the minimum amount of antibiotic required to completely kill planktonic and biofilm cultures, respectively (Andrews, 2002; Ceri et al., 2001). However, there are differing environmental and physiological factors to consider when comparing the antibiotic susceptibility of biofilms to planktonic cultures. In order to compare the two systems directly all factors should be equal.

A drawback to the MBEC method of antibiotic susceptibility testing is that it is destructive as the experiment needs to be stopped in order to determine the biofilms MBEC. For this dissertation, there was interest in monitoring a biofilm's response to antibiotics in real-time and non- 
destructively. In addition, since many studies already look at genetics and MIC and MBEC studies, it was of interest to monitor biofilm metabolism in response to antibiotics to determine if metabolic studies coincide with results obtained by other methods.

\subsubsection{Real-Time Monitoring of Antimicrobial Effects on Biofilms}

As mentioned earlier, biofilms are responsible for a large number of infections. Traditional antibiotic susceptibility testing such as planktonic MIC tests has not always been useful in determining biofilm susceptibility to high concentrations of antimicrobials. Fortunately, a few groups have come up with unique way to monitor biofilms in real-time that can help delineate bacterial behaviour during antimicrobial exposures.

Firstly, a $\mathrm{CO}_{2}$ evolution measurement system (CEMS) that measures gas phase $\mathrm{CO}_{2}$ produced by whole-biofilms (Kroukamp and Wolfaardt, 2009) is a tool that can be used to assess the effects of antimicrobials on biofilm metabolism (Fig. 1.1). The CEMS can be used non-destructively and in real time while monitoring biofilm antimicrobial response and recovery. The CEMS setup allows for the growth of both aerobic and anaerobic biofilms (Kroukamp et al., 2010). Environmental parameters can easily be changed without disrupting the biofilm. Given that the measurements can be taken without disrupting the biofilm, it is possible to observe recovery of the biofilm. This occurs even days after an antimicrobial exposure appeared to completely kill the biofilm based on viable counts or $\mathrm{CO}_{2}$ respiration levels.

A second real-time biofilm measurement system that was developed to monitor physiological states of biofilms is chip calorimetry. The chip calorimetry tool measures heat production of biofilms, which can be monitored during antimicrobial exposures (Buchholz et al., 2010a). Chip calorimetry is beneficial because it, similarly to CEMS, does not interfere with or disrupt the biofilm. As long as every known chemical and physical process is taken into account, as they can also absorb or release heat, data can be interpreted directly (Buchholz et al., 2010b).

For this study, the CEMS system was chosen in order to monitor biofilm $\mathrm{CO}_{2}$ production, because in combination with other methods and tools, real-time monitoring of biofilm behaviour can provide insight into the mechanisms involved in biofilm antimicrobial resistance. Given that many cellular processes require energy and in turn can affect antibiotic susceptibility, it seemed fitting to monitor the effects of antibiotics on biofilms through monitoring of metabolism. 
Metabolic analysis of $\mathrm{CO}_{2}$ production in biofilms, planktonic and effluent MIC testing, microscopy, effluent viable cell counts, and DNA work were methods used to confirm antibiotic susceptibility and resistance.
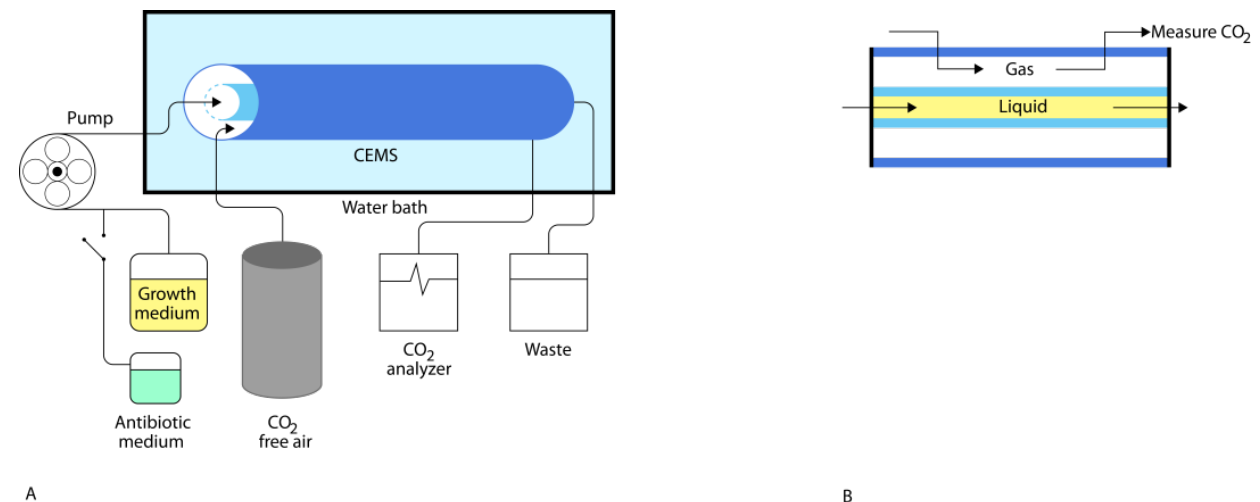

Figure 1.1. Monitoring of biofilm metabolism through the capture of $\mathrm{CO}_{2}$ production in real-time. (A) The CEMS system was setup with media being fed into a gas-permeable inner silicone tube in the CEMS using a peristaltic pump. $\mathrm{CO}_{2}$ released by biofilms in the inner tube cross the silicon wall into the annular space confined by a gas-impermeable outer tygon tube, from where it is collected and transferred by $\mathrm{CO}_{2}$-free sweeper gas to a $\mathrm{CO}_{2}$ analyzed. (B) A cross-section of the CEMS showing flow of growth medium (liquid) and gas, separated by a gas-permeable silicone.

\subsection{Relevant Methods of DNA Analysis}

\subsubsection{Polymerase Chain Reaction and Genomic Analysis}

In addition to monitoring bacterial and planktonic behavioural responses to antibiotics, genetic analysis can be performed in order to determine the genetic potential of bacterial cultures and biofilms. Both polymerase chain reaction (PCR) and genomic testing can be performed on bacterial biofilms and their planktonic counter parts providing useful information regarding potential antimicrobial resistance mechanisms. Many resistance mechanisms develop from the acquisition of antibiotic resistance plasmids and genetic mutations which alter antibiotic uptake, efflux, or cause changes in antimicrobial targets (Moore and Flaws, 2011).To detect the presence of a gene of interest PCR can be used to rapidly amplify segments of DNA using RNA primers (Erlich et al., 2015) and detect the presence of antibiotic resistance genes that are carried on plasmids or integrated into the bacterial genome (e.g. aminoglycoside modifying enzymes) (Kaito et al., 2009). Genomic analysis is a top down approach that provides a contiguous sequence of all the chromosomes in a bacterium. For example, genome sequencing can provide information on the set of virulence or resistance factors present in a bacterium (Weinstock, 2000). 


\subsubsection{Overview of mg-RAST (metagenomic-Rapid Annotation using Subsystem Technology) method of genome analysis}

Genomic and metagenomic analyses are often needed by researchers to study microorganisms

for a multitude of purposes. Sequencing bacterial genomes has become more efficient with the use of bench top sequencers, such as Illuminas MiSeq, Ion Torrent PGM, or Roche 454 FLX Junior (Edwards and Holt 2013). Once a genome sequence has been generated it must be compared to known sequence databases for further analysis and annotations (Meyer et al., 2008). An increase in the number of configuration and analysis tools that are available for researchers to use has decreased challenges with genome analysis. RAST is one of the open-source metagenomic tools that allows for annotation of genomic data. Once a researcher has a genome that is sequenced and is in the form of a FASTA, FASTQ, or SFF file, RAST can annotate the sequence. The mg-RAST server compares metagenome sequence data using the SEED framework (Meyer et al., 2008). The SEED is a website service that is continually updating its genomic data, genome annotations, functionally related proteins and protein families from a variety of sources. As RAST annotates a new genome, it cross-checks information provided on the SEED server (Overbeek et al., 2013).

\subsection{Purpose of the Proposed Research Project}

In this dissertation it was shown how changes in environmental conditions and energy status can affect biofilm susceptibility to high doses of antibiotics. Changes in carbon concentration can alter growth state and energy availability, while changes in $\mathrm{pH}$ affect the net charge on aminoglycosides and the electrical potential. These environmental factors invariably affect aminoglycoside efficacy. This study focused on the monitoring of whole-biofilm behaviour during high dose aminoglycoside antibiotic exposures at various $\mathrm{pH}$ and with added carbon as these environmental conditions affect antibiotic uptake, energy-requiring antibiotic resistance mechanisms, and biofilm physiological status. In addition, genetic analysis was performed to analyze the intrinsic potential of various cultures tested in this study.

\subsection{Outline of Chapter 2}

The first goal was to delineate the metabolic response of early steady state biofilms grown in CEMS when exposed to high concentrations of streptomycin. Next, there was interest in 
determining how antibiotic susceptibility changed upon the addition of carbon to the antibiotic media, during streptomycin exposure, since streptomycin resistance and uptake can require energy. Monitoring of metabolism before, during and after antibiotic exposures allowed us to make further hypotheses regarding mechanisms of biofilm survival. The CEMS is a tool that allows for monitoring of whole-biofilm behaviour and is unique in regards to the fact that it captures $\mathrm{CO}_{2}$ metabolism non-destructively, in real-time.

Initially, high concentration streptomycin exposures were performed on multispecies biofilms containing predominantly the nosocomial pathogens $P$. aeruginosa and $S$. maltophilia. $P$. aeruginosa and $S$. maltophilia are easily accessible pathogenic bacteria that have been shown to resist high concentrations of antimicrobials (Nithya et al., 2010; Brooke, 2012). The intentions of this work were not to solve medically relevant ways to treat biofilm infections, but to gain insight into the behaviour of common pathogens when they are treated with high doses of antibiotics. The experiments demonstrated, under certain conditions, the biofilms do not survive the high dose antibiotic exposures. In particular, when the mixed species biofilms were exposed to high doses of streptomycin at early steady state in $0.3 \mathrm{~g} / \mathrm{L} \mathrm{TSB}$ medium (containing $0.14 \mathrm{mM}$ glucose) they would be susceptible to the antibiotic treatment. The addition of extra carbon during the exposures could aid the biofilms in recovering from the antibiotic. Furthermore, when a defined medium was used with citrate as the carbon at the same concentration $(0.14 \mathrm{mM})$ as the $0.3 \mathrm{~g} / \mathrm{L}$ TSB medium, biofilms were able to recover from the high dose streptomycin exposures. Thus, in the defined $0.14 \mathrm{mM}$ citrate medium the biofilms were less susceptible than in the 0.3 g/L TSB medium.

Further testing was performed on pure culture Gram-negative bacteria and the multispecies culture stored as a bench culture. The other bacterial strains that were exposed to high doses of streptomycin in $0.3 \mathrm{~g} / \mathrm{L}$ TSB in CEMS included: 3 Gram-negative environmental isolates $(E$. asburiae, Enterobacter sp., and $P$. putida), a pure culture of $P$. aeruginosa PAO1 and PAO1 $\Delta \mathrm{MexXY}$, and a multispecies bench culture. Unlike the multispecies culture, all the environmental isolates and the bench culture were able to recover from the high dose antibiotic exposures. However, the pure PAO1 and PAO1 $\triangle \mathrm{MexXY}$ mutant were unable to recover from the high dose streptomycin exposures in $0.3 \mathrm{~g} / \mathrm{L}$ TSB medium when inoculated directly from a freezer stock culture, but when the PAO1 pure culture was inoculated from a bench culture it 
could recover from the high dose streptomycin exposures with and without the addition of carbon.

Analysis of biofilm metabolic behaviour following antibiotic exposures provided useful information in terms of antibiotic susceptibility of the various cultures to streptomycin antibiotics. Particular trends in metabolism when the various Gram-negative isolates were exposed to streptomycin were delineated.

\subsection{Outline of Chapter 3: The influence of $\mathrm{pH}$ whole-biofilm susceptibility to gentamicin and streptomycin as monitored by $\mathrm{CO}_{2}$ production}

Aminoglycoside antibiotics are positively charged and their uptake is potentiated by an electrical potential difference across the bacterial membrane that leaves the inner cytoplasmic membrane negatively charged (Shapiro, 2000; Taber, 1987; Damper, 1981). Owing to the fact $\mathrm{pH}$ can affect the overall charge on aminoglycosides and the electrical potential across bacterial membranes (Damper, 1981; Whooley and McLoughlin, 1983) it was of interest to test how biofilm metabolism and subsequent susceptibility to aminoglycoside antibiotics would be affected at different $\mathrm{pH}$.

To monitor biofilm susceptibility to aminoglycosides at various $\mathrm{pH}$, the aminoglycosides gentamicin and streptomycin were used on biofilms grown from a multispecies culture containing primarily $P$. aeruginosa and S. maltophilia. Biofilms were grown in $0.3 \mathrm{~g} / \mathrm{L} \mathrm{TSB}$ at $\mathrm{pH} 7.0$ in the CEMS apparatus to steady state levels prior to being exposed to $4000 \mathrm{mg} / \mathrm{L}$ of the aminoglycosides in $0.3 \mathrm{~g} / \mathrm{L} \mathrm{TSB}$ medium at $\mathrm{pH} 6.0,7.0$, or 7.4. Biofilm effluent samples were collected before, during and after the antibiotic exposures to determine viable cell counts. In addition, effluent antibiotic MICs were monitored before exposures and following recovery from an exposure to track changes in antibiotic susceptibility. The CEMS can only track changes in biofilm metabolism, but does not provide information on biofilm structure and density. Therefore, biofilms were grown in flow cells so that they could be analyzed microscopically following antibiotic exposures. Live/dead staining was performed on biofilms following gentamicin exposures at either $\mathrm{pH} 6.0$ or $\mathrm{pH} 7.4$ and the effects of the antibiotics on biomass were observed. 
Monitoring the effects of aminoglycosides and $\mathrm{pH}$ on biofilm behaviour and antibiotic susceptibility helped to gain insight into which conditions most adversely affected the biofilm. Though both gentamicin and streptomycin are aminoglycosides, there were differences in biofilm susceptibility to the antibiotics at the $\mathrm{pH}$ tested.

\subsection{Outline of Chapter 4: The intrinsic potential of the planktonic and biofilm cultures used in this study}

Behavioural analysis of bacterial biofilms can provide useful information on biofilm response to antibiotics, however, inevitably questions regarding the mechanisms of how biofilms are able to resist antibiotics will arise. Several methods were used to determine the intrinsic potential that the bacterial strains used in this study had for streptomycin or gentamicin resistance. This included PCR for all possible streptomycin-modifying enzymes in each culture tested and metagenomic analysis of the multispecies culture in various physiological states and following antibiotic exposures in biofilm. The overall goal of looking for streptomycin modifying enzymes with PCR and doing full genome analysis was to determine potential mechanism of antibiotic resistance that the bacterial cultures could employ to resist doses of antibiotics 100 to1000 times greater than their planktonic MIC.

For PCR studies, genomic DNA of the 3 Gram-negative environmental isolates (E. asburiae, Enterobacter sp., and P. putida) as well as the multispecies cultures was amplified with streptomycin modifying enzymes. In addition, full genome sequencing was performed on the multispecies cultures in planktonic state, from a biofilm, and biofilm effluent before and after streptomycin exposures. The annotated genomes were scoured for any genes or genetic mutations that could have lead to the intrinsic resistance of the antibiotics. One streptomycin modifying enzyme was identified in P. putida from the PCR work and several aminoglycoside modifying enzymes were found from the genomic and metagenomic analysis performed on the sequenced genomes. In addition, metagenomic analysis also revealed shifts in community composition of the multispecies culture under the conditions tested. P. aeruginosa was the dominant species in planktonic cultures and in the defined medium. While S. maltophilia increased its percentage in the population when going from a planktonic state to biofilm and became the dominant species following a streptomycin exposure in the complex medium. 
Although the presence of a gene in a culture does not necessarily result in upregulation of that gene, knowing the intrinsic potential can help infer potential mechanisms of antibiotic resistance. Further research can then be targeted to look into the specific genes that may be upregulated in order to benefit the bacteria in the presence of an antibiotic. Future studies can also be performed on how and when members of a bacterial culture provide benefit to the community as a whole. 


\section{Chapter 2}

\section{Biofilm-Metabolic Response to Streptomycin is Dependent upon Carbon Source and/or Concentration during Exposure}

*This chapter has been published as: Jackson, L., Kroukamp, O., and Wolfaardt, G.M. 2015. Effect of Carbon on Whole-Biofilm Metabolic Response to High Doses of Streptomycin. Front. Microbiol., 6:953. doi: 10.3389/fmicb.2015.00953 (Writing was completed in consultation with the co-authors.) 


\section{Biofilm-Metabolic Response to Streptomycin is Dependent upon Carbon Source and/or Concentration during Exposure}

\subsection{Abstract}

Biofilms behave as complex communities made of multiple species with the ability to adapt to a variety of harsh conditions. In clinical settings, antibiotic treatments based on planktonic susceptibility tests are often ineffective against biofilm infections, as biofilms can withstand much higher antibiotic concentrations. Biofilm responses to doses of antibiotic much greater than their planktonic susceptibilities were analyzed. Using a $\mathrm{CO}_{2}$ evolution measurement system we delineated the real time metabolic response to streptomycin exposures in continuous flow biofilms. Metabolically stable biofilms from a multispecies culture (containing mainly Pseudomonas aeruginosa and Stenotrophomonas maltophilia), biofilms grown from Gramnegative environmental isolates, and biofilms grown from pure culture $P$. aeruginosa strains

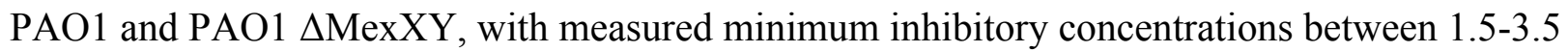
$\mathrm{mg} / \mathrm{L}$, were exposed to $4000 \mathrm{mg} / \mathrm{L}$ streptomycin for $4 \mathrm{~h}$ after which sterile growth medium was resumed. In complex growth medium, early steady state multispecies biofilms were susceptible to streptomycin exposures, inferred by a cessation of $\mathrm{CO}_{2}$ production. However, the multispecies biofilms were able to survive the high dose exposures when extra carbon was in the complex growth medium with antibiotic medium, or when they were grown in a defined citrate medium. The single species environmental strains and PAO1 biofilms showed similar metabolic profiles in response to the antibiotic; ceasing $\mathrm{CO}_{2}$ production after initial exposure, with $\mathrm{CO}_{2}$ levels

dropping towards baseline levels prior to recovery back to steady state levels, followed by subsequent antibiotic exposures with increased $\mathrm{CO}_{2}$ output above initial steady state values. The ability to monitor biofilm metabolic responses in real-time allowed us to explore the environmental conditions resulting in early vulnerability after antibiotic exposure compared to the resistance displayed subsequent to additional exposures.

\subsection{Introduction}

Most biofilms are formed by multispecies microbial communities (Lindsay and Holy, 2006). Biofilms have many positive roles in nature (e.g. recycling of elements in soils), industry (e.g. wastewater treatment), and for human health (e.g. intestinal nutrient metabolism) though they 
may cause negative effects such as corrosion of oil rigs and pipelines and a variety of infections in humans (Lavelle et al., 2006; Qureshi et al., 2005; Flint et al., 2007; Gottenbos et al., 1999). More than $65 \%$ of bacterial infections in humans are caused due to bacteria forming biofilms and $10-20 \%$ of nosocomial infections are caused by the formation of biofilms on medical devices (Nithya et al., 2010). Once a biofilm has formed in a chronic wound it is difficult for the host's immune system to eradicate (Fux et al., 2005), and thousands of deaths and billions of dollars in medical costs are incurred each year from such infections (Dowd et al., 2008). A variety of bacterial species are often isolated from biofilm infections, with two common pathogens associated with nosocomial infections being Stenotrophomonas maltophilia and Pseudomonas aeruginosa (Dowd et al., 2008; Tseng et al., 2009; Pedersen et al., 1992; Nithya et al., 2010). Stenotrophomonas and Pseudomonas species are associated with a number of illnesses and have been co-isolated in several wound types and infections (Araoka, 2010; Tseng et al., 2008).

With a large proportion of infections caused by bacterial biofilms, many efforts have been made to understand antibiotic resistance, as antibiotics remain a main treatment of bacterial infections (Hanlon, 2007; Martin and Ernst, 2003). Earlier reports indicated that bacterial biofilms can resist concentrations of antibiotics up to 1000 times greater than their planktonic counterparts (Nithya, 2010; Mah and O'Toole, 2001; Nickel, 1985; Gristina et al., 1987) though there are studies that point out this is a false impression (Spoering and Lewis, 2001). Biofilm antibiotic resistance has been linked to various conditions and behaviours specific to the biofilm environment, such as reduced antibiotic penetration due to adsorption to matrix components or degradation by extracellular enzymes (Stewart and Costerton, 2001), slower growth (Ashby et al., 1994), expression efflux pumps (Brooun et al., 2000), expression and/or increased local concentrations of antibiotic-modifying or -degrading enzymes (O'Toole et al. 2000; Giwercman et al., 1991), alterations in antibiotic targets (O'Toole et al., 2000), nutrient limitation (Dorrer and Teuber, 1977; Nguyen et al., 2011), adaptive stress responses (Nguyen et al., 2011), and the presence of persister cells (Spoering and Lewis, 2001; Stewart, 2002).

Although having antibiotic resistance genes is important for bacterial resistance, the physiological state of bacteria can greatly affect their susceptibility since antibiotics traditionally target various forms of macromolecular synthesis (Cotsonas and Wu, 2009; Hancock, 1981; Eng et al., 1991; Hooper, 2001). Thus, it can be expected that nutrients and energy sources affect 
bacterial susceptibility to antimicrobials. Earlier work with $P$. aeruginosa demonstrated that the minimum inhibitory concentration (MIC) of colistin and polymyxin B was dependent on the carbon source in which the bacteria were cultivated (Conrad, 1979). Another study found that carbon sources entering in upper glycolysis (such as glucose and mannitol) potentiate aminoglycoside killing of bacterial persister cells as compared to carbon sources that entered lower glycolysis (such as succinate or citrate) (Allison et al., 2011). Furthermore, efflux pump mediated resistance in clinically relevant Gram-negative bacteria can be reduced by substituting the culture medium with non-phosphotransferase system sugars that cause changes in protein concentration in the periplasmic space, which limit efflux pump activity (Villagra, 2012).

The preceding studies described how various carbon sources affect antibiotic susceptibility by investigating how variations in substrate can affect bacterial growth, antibiotic uptake, and cellular composition in planktonic systems. Since bacterial biofilms are highly resistant to antibiotics, how whole-biofilm metabolism and antibiotic susceptibility were affected when grown in various carbon sources and when additional carbon was added during antibiotic exposure was explored. The antibiotic streptomycin was used, which is an aminoglycoside that targets protein synthesis, and can disrupt the integrity of the bacterial outer membrane (Taber et al., 1987). Both streptomycin uptake and efflux are energy-dependent processes (Taber et al., 1987; Webber and Piddock, 2003), and certain antibiotic modifying enzymes may require energy, which could all be affected by nutritional status. Thus, energy dependent processes and the net flux of the antibiotic could influence the survival. Finding the mechanisms of antibiotic resistance was not the primary objective of this study, though an efflux pump mutant was subjected to the same conditions to determine if a similar response would be observed. Through careful monitoring of whole-biofilm metabolic response to high doses of antibiotics in various nutrient conditions there was interest in demonstrating important aspects of biofilm behaviour that can be further explored with gene, protein, or cellular based methods.

To test how changes in medium and carbon content affect biofilm susceptibility, the $\mathrm{CO}_{2}$ evolution measurement system (CEMS) (Kroukamp and Wolfaardt, 2009) was used. The goal was to delineate the real-time metabolic response of whole biofilms of a Stenotrophomonas maltophilia-P. aeruginosa containing multispecies culture, as well as several environmental isolates when exposed to high concentrations of streptomycin in complex and defined growth 
media with varying carbon concentrations. Genomic analyses of the starting cultures obtained from freezer stocks and a bench culture both revealed the presence of S. maltophilia, despite the frequent subculturing of the bench culture on agar plates. Further metagenomic analysis was performed on biofilm and biofilm effluent (before and after antibiotic exposure), which showed the presence of $S$. maltophilia in the cultures as well. Streptomycin was applied at concentrations a 100 to a 1000 times greater than the planktonic minimum inhibitory concentration (MIC), in accordance with the frequent reference to these high values in the literature (e.g., Mah and O’Toole, 2001). Increases in biofilm metabolism upon antibiotic addition were observed. Therefore, it was hypothesized that the addition of carbon during antibiotic exposures may aid in biofilm recovery from high dose streptomycin exposures, in contrast to the notion that biofilms survive high concentration antibiotic exposures due to inactivity. Keeping in mind that biofilms are spatially highly heterogeneous, the aim was to elucidate trends in whole-biofilm metabolic behaviour.

\subsection{Materials and Methods}

\subsubsection{Bacterial cultures}

Three Gram-negative environmental strains (Enterobacter asburiae, Enterobacter sp., and Pseudomonas putida) isolated from a washroom sink drain (Ghadakapour et al., 2014), and a multispecies culture containing Pseudomonas aeruginosa and Stenotrophomonas maltophilia were used in this study to compare metabolic behaviour among Gram-negative isolates exposed to streptomycin antibiotics. The bacteria in the multispecies biofilm containing $P$. aeruginosa and $S$. maltophilia were determined by full genome sequencing and metagenomic analysis and the environmental strains were identified by $16 \mathrm{~S}$ rRNA. Further testing was done on single species biofilms of P. aeruginosa strains PAO1 and PAO1 $\triangle \mathrm{MexXY}$ (Fraud and Poole, 2011) to observe how efflux pump activity affects response to high dose streptomycin exposures. Continuous flow systems (see below) were inoculated from freezer stocks as well as bench cultures repeatedly sub-cultured on agar plates. All pre-cultures were grown in either $3 \mathrm{~g} / \mathrm{L}$ tryptic soy broth (TSB, EMD Chemicals, Billerica, MA, USA.) (which contains $1.4 \mathrm{mM}$ glucose) or in a defined growth medium with final concentrations of $1.51 \mathrm{mM}\left(\mathrm{NH}_{4}\right)_{2} \mathrm{SO}_{4}, 3.37$

$\mathrm{mM} \mathrm{Na}_{2} \mathrm{HPO}_{4}, 2.20 \mathrm{mM} \mathrm{KH}_{2} \mathrm{PO}_{4}, 179 \mathrm{mM} \mathrm{NaCl}, 0.1 \mathrm{mM} \mathrm{MgCl} 2 \cdot \mathrm{H}_{2} \mathrm{O}, 0.01 \mathrm{mM} \mathrm{CaCl} \cdot 2 \mathrm{H}_{2} \mathrm{O}$, 
and $0.001 \mathrm{mM} \mathrm{FeCl}_{3}$ with $5 \mathrm{mM}$ glucose or sodium citrate (Clark and Maaløe, 1967) at 37 C, while agitated (300 rotations per minute (rpm)).

\subsubsection{Antibiotic minimum inhibitory concentration (MIC)}

A stock solution of streptomycin sulfate (Bio Basic Inc. Markham, ON, CA) with a final concentration of $10,000 \mathrm{mg} / \mathrm{L}$ was prepared following a protocol described earlier (Andrews, 2001). The MIC of streptomycin for the multispecies culture containing P. aeruginosa and $S$. maltophilia strains, E. asburiae, Enterobacter sp., and $P$. putida were determined in $3 \mathrm{~g} / \mathrm{L}$ TSB (Ghadakapour et al., 2014). In addition, biofilm effluent MIC was obtained for multispecies biofilms inoculated from bench cultures (see biofilm effluent collection described below and Fig. 4A, B). MIC's were determined at $25{ }^{\circ} \mathrm{C}$ for all isolates, as well as at $37{ }^{\circ} \mathrm{C}$ for the multispecies culture. Although MIC's are generally performed at $37{ }^{\circ} \mathrm{C}$, testing at $25{ }^{\circ} \mathrm{C}$ was performed in order to demonstrate the planktonic antibiotic susceptibility at the same temperature used for biofilm antibiotic testing. Approximately $10^{7}$ cells ( $100 \mu \mathrm{l}$ from a $10^{8}$ cells $/ \mathrm{ml}$ suspension) of overnight culture were added to the $5 \mathrm{ml}$ antibiotic dilutions and incubated overnight with shaking at $300 \mathrm{rpm}$, at either $37{ }^{\circ} \mathrm{C}$ or $25{ }^{\circ} \mathrm{C}$ for $18-20 \mathrm{~h}$. The antibiotic concentrations tested ranged from 1.75 - $200 \mathrm{mg} / \mathrm{L}$ streptomycin and each dilution tested was performed in triplicate. A positive control $\left(10^{7}\right.$ cells from the overnight culture added to sterile $3 \mathrm{~g} / \mathrm{L}$ TSB without antibiotic) and a negative control (sterile $3 \mathrm{~g} / \mathrm{L}$ TSB medium without any inoculum) were prepared for each experiment. The antibiotic MIC was determined at the concentration of antibiotic that resulted in no growth of the culture (as determined by no turbidity or cloudiness seen in the culture) (Andrews, 2001).

\subsubsection{Biofilm development}

A carbon dioxide evolution measurement system (CEMS) was used to grow biofilms. In this system, a continuous flow of growth medium is fed into inner silicone tubing where the biofilm grows. The silicone tubing is permeable to gas enabling the $\mathrm{CO}_{2}$ produced by the biofilm to be collected by a $\mathrm{CO}_{2}$-free sweeper gas and measured in real time through a $\mathrm{CO}_{2}$ analyzer (Kroukamp et al., 2010). Growth medium, with and without added antibiotic, was fed into the CEMS at a flow rate of $15 \mathrm{ml} / \mathrm{h}$ (hydraulic retention time of $8 \mathrm{~min}$ ) using a peristaltic pump. Planktonic cells were being washed away faster than they can multiply within the tube because 
the dilution rate exceeds planktonic bacterial specific growth rates by at least 10 times. The CEMS apparatus was immersed in a water bath kept at $25{ }^{\circ} \mathrm{C}$. Biofilms were fed continuously with $0.3 \mathrm{~g} / \mathrm{L}$ TSB medium until biofilms reached metabolic levels that corresponded with late exponential phase, early steady state, or late steady state levels prior to the aminoglycoside exposures. For inoculation, one milliliter of the respective pre-cultures was introduced into the CEMS without flow for up to 60 minutes before flow of media was resumed. Batch-grown precultures were always cultivated in the same complex (TSB) or defined medium as the biofilm under investigation.

For the purpose of the biofilm experiments, low and high TSB concentrations were considered to be $0.3 \mathrm{~g} / \mathrm{L}$ ( $1 \%$ of manufacturer's recommended concentration containing $0.14 \mathrm{mM}$ glucose) and $3 \mathrm{~g} / \mathrm{L}$ (1.4 mM glucose), respectively. In the case of the defined medium, high concentrations of carbon were considered to be at $1 \mathrm{mM}$ for citrate and glucose or $2 \mathrm{mM}$ for pyruvate added to the antibiotic medium, while citrate used at a concentration of $0.14 \mathrm{mM}$ was considered a low carbon medium.

\subsubsection{Antibiotic exposure}

The $\mathrm{CO}_{2}$ profile produced by biofilms grown in the CEMS was used to determine when the biofilms had reached a metabolically-stable state between $24-48 \mathrm{~h}$ after inoculation (early steady state biofilms). Streptomycin sulfate (Biobasic Inc. Markham, ON, CA) was added directly to sterile medium and the biofilms were exposed to streptomycin concentrations of $4000 \mathrm{mg} / \mathrm{L}$ up to $12000 \mathrm{mg} / \mathrm{L}$ for $4 \mathrm{~h}$; the physiological half-life of streptomycin in human plasma is $2-4 \mathrm{~h}$, thus a 4-h exposure time was chosen (Clarke, 1986). All of the antibiotic exposures were performed at a minimum in duplicates, except for the antibiotic exposures on the two environmental isolates E. asburiae and Enterobacter sp.

\subsubsection{Viable cell counts in biofilm effluent}

Effluent samples were collected to determine the viability and numbers of planktonic cells being released from biofilms before, during, and after antibiotic exposures. Biofilm effluent samples collected from steady state biofilms fed with antibiotic-free growth medium were serially diluted before plating. Biofilm effluent samples collected from antibiotic containing medium were 
washed twice via centrifugation at $12000 \mathrm{x}$ g for 150 seconds and re-suspended in $0.9 \%$ saline solution to reduce the presence of residual antibiotic before plating on $3 \mathrm{~g} / \mathrm{L}$ TSA plates and incubated at $37^{\circ} \mathrm{C}$.

\subsection{6 pH controls}

The $\mathrm{pH}$ of the media used in this study ranged from 6.71-7.09, and the $\mathrm{pH}$ of the antibiotic containing media ranged between 6.14-6.36. Lower $\mathrm{pH}$ increases the ratio of dissolved $\mathrm{CO}_{2}$ to bicarbonate ions. Since it is the dissolved $\mathrm{CO}_{2}$ that crosses the silicone tube wall it could be argued that sudden increases in measured $\mathrm{CO}_{2}$ upon a decrease in $\mathrm{pH}$ could be attributed to this $\mathrm{pH}$ (and dissolved $\mathrm{CO}_{2}$ ratio) shift alone. Even for any $\mathrm{pH}$ where the bicarbonate ion to dissolved $\mathrm{CO}_{2}$ ratio may increase, the entire reactor volume would still be replaced within 8 minutes, therefore any measured $\mathrm{CO}_{2}$ changes due to $\mathrm{pH}$ induced dissolved $\mathrm{CO}_{2}$ ratio would be transient.

\subsection{Results}

\subsubsection{Antibiotic susceptibility of mixed species biofilms grown in $0.3 \mathrm{~g} / \mathrm{L}$ TSB medium}

To test the notion that biofilms can withstand antibiotic concentrations 10 to a 1000 times their planktonic MIC, multispecies-biofilms (inoculum planktonic MIC between 1.5-3.5 mg/L) were exposed to $4000 \mathrm{mg} / \mathrm{L}$ of streptomycin. As previously observed (Kroukamp et al., 2010), biofilms formed by Pseudomonas strains may have notably different lengths in lag phase that will cause the biofilm to stabilize $\mathrm{CO}_{2}$ production at different times depending on the inoculum. Thus biofilm growth stage was considered as criterion for when to introduce the antibiotic - a factor deserving to be considered in biofilm research. Biofilms grown until just before the onset of steady state and early steady state $\mathrm{CO}_{2}$ production were susceptible to $4000 \mathrm{mg} / \mathrm{L}$ of streptomycin (Fig. 1A1-A3). Even when provided with antibiotic-free growth medium for up to 7 days following exposure, the biofilms did not recover.

\subsubsection{Added carbon during antibiotic exposure on young biofilms grown in $0.3 \mathrm{~g} / \mathrm{L}$ TSB medium (containing $0.14 \mathrm{mM}$ glucose)}

Biofilms exposed to high doses of streptomycin (Fig. 2.1A1-A3) demonstrated a brief spike in $\mathrm{CO}_{2}$ production upon addition of the antibiotic medium. This innate tendency to increase 
metabolism at the onset of streptomycin addition led to the hypothesis that added energy during an antibiotic exposure, especially for early steady state biofilms, would decrease susceptibility of biofilms grown in low concentration $(0.3 \mathrm{~g} / \mathrm{L}) \mathrm{TSB}$. This was demonstrated (similar to biofilms shown in Fig. 2.1A2-A3) by providing additional carbon during the antibiotic exposure. Indeed, when biofilms grown in $0.3 \mathrm{~g} / \mathrm{L}$ TSB were exposed to $4000 \mathrm{mg} / \mathrm{L}$ streptomycin dissolved in high concentration $(3 \mathrm{~g} / \mathrm{L}) \mathrm{TSB}$, there was an immediate spike in metabolism, followed by a drop in $\mathrm{CO}_{2}$ production to near baseline levels and subsequent recovery over a period of more than $100 \mathrm{~h}$ to return to steady state (Fig. 2.1B1). Similarly, the biofilms grown in $0.3 \mathrm{~g} / \mathrm{L} \mathrm{TSB}$ could be rescued when exposed to streptomycin with the addition of $1 \mathrm{mM}$ glucose or $2 \mathrm{mM}$ pyruvate (Fig. 2.1B2-B3). Unlike glucose or pyruvate addition, addition of $1 \mathrm{mM}$ citrate to the antibiotic medium did not result in the rescue of the biofilm (Fig. 1A4). When comparing the graphs in column A to the graphs in column B there are a few observations worth mentioning: In column B, when additional carbon sources are added to the antibiotic media (Fig. 1) the initial increases in $\mathrm{CO}_{2}$ output are greater than those in column $\mathrm{A}$ when no carbon is added along with the antibiotic, or when citrate is added to the antibiotic medium. Furthermore, the $\mathrm{CO}_{2}$ outputs of the biofilms in column B (Fig. 2.1B1-B2) do not drop completely to base line levels prior to biofilm recovery towards steady state $\mathrm{CO}_{2}$ output. 

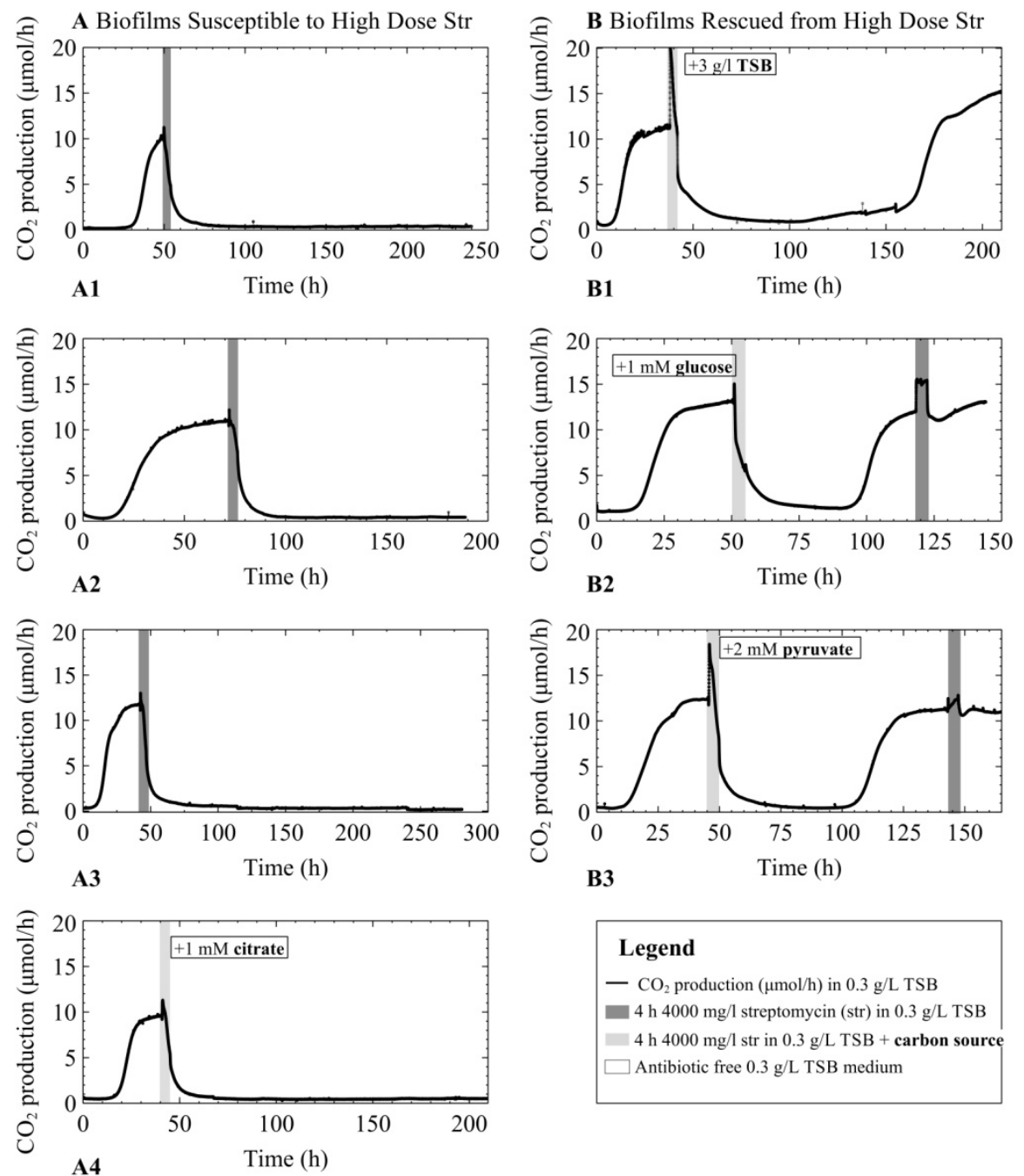

Figure 2.1. The $\mathrm{CO}_{2}$ production $(\mu \mathrm{mol} / \mathrm{h}$ ) of multispecies biofilms grown on $0.3 \mathrm{~g} / \mathrm{L} \mathrm{TSB}$ medium exposed to $4000 \mathrm{mg} / \mathrm{L}$ streptomycin with and without the addition of carbon to the antibiotic medium. Column $A$ delineates late exponential phase (A1) and early steady state biofilms (A2-A4) exposed to streptomycin. The biofilms were exposed to streptomycin in either 0.3 $\mathrm{g} / \mathrm{L} \mathrm{TSB}$ medium ( $\mathrm{A} 1-\mathrm{A} 3$ ) or $0.3 \mathrm{~g} / \mathrm{L}$ TSB medium with the addition of $1 \mathrm{mM}$ citrate to the $0.3 \mathrm{~g} / \mathrm{L} \mathrm{TSB}$ antibiotic medium (A4). Column $B$ delineates the metabolic response of biofilms that recovered following the high dose streptomycin exposures from the addition of either $3 \mathrm{~g} / \mathrm{L}$ TSB (B1), $1 \mathrm{mM}$ glucose (B2), or $2 \mathrm{mM}$ pyruvate (B3) to the antibiotic medium.

\subsubsection{Effect of additional carbon during antibiotic exposures on biofilms grown in low carbon $(0.14 \mathrm{mM})$ defined medium}

Bacteria behave differently when provided with various nutrient sources, in either complex or minimal media (Rojo, 2010). In addition, $P$. aeruginosa prefers organic acids (as carbon sources) to glucose (Ng and Dawes, 1967). Thus to how the multispecies biofilms survive antibiotic exposures with citrate as the carbon source the multispecies biofilms were grown in a defined medium with citrate as the sole carbon source (at a concentration equal to that of the glucose 
$(0.14 \mathrm{mM})$ in the $0.3 \mathrm{~g} / \mathrm{L}$ TSB medium) would show similar sensitivity to the antibiotic to when grown in $0.3 \mathrm{~g} / \mathrm{L}$ TSB medium.
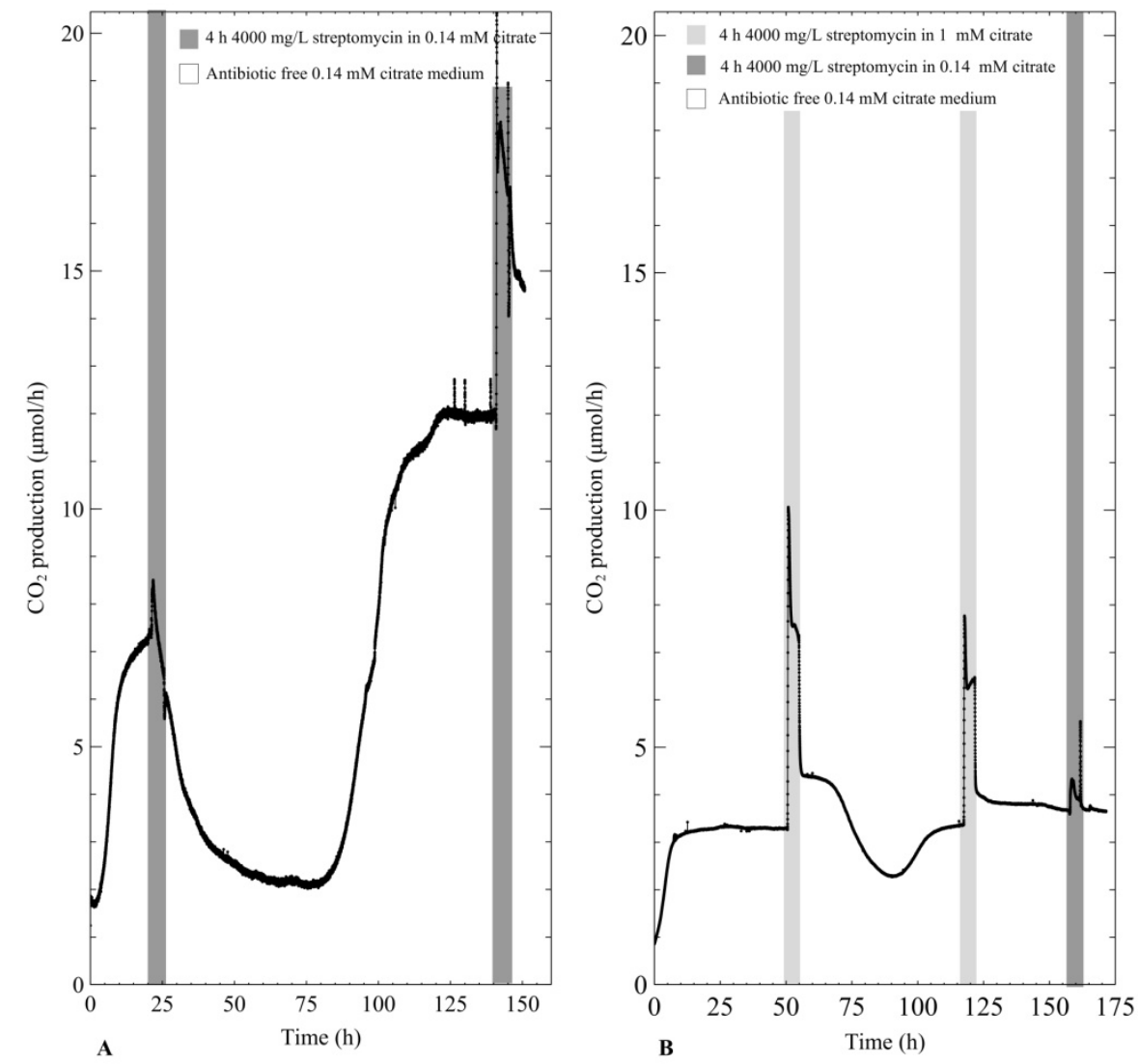

Figure 2.2. The $\mathrm{CO}_{2}$ production ( $\mu \mathrm{mol} / \mathrm{h}$ ) of multispecies biofilms grown in $0.14 \mathrm{mM}$ citrate defined medium and exposed to $4000 \mathrm{mg} / \mathrm{L}$ streptomycin for 4-h intervals (A) $0.14 \mathrm{mM}$ citrate medium (B) $0.14 \mathrm{mM}$ citrate growth medium with the addition of $1 \mathrm{mM}$ citrate during the first and second exposure, and a third antibiotic exposure in $0.14 \mathrm{mM}$ citrate medium.

The metabolic profile of early steady state multispecies inoculum biofilms grown in low concentration $(0.14 \mathrm{mM})$ citrate medium showed a slight increase in $\mathrm{CO}_{2}$ production upon antibiotic exposure before $\mathrm{CO}_{2}$ levels decreased towards baseline levels (Fig. 2.2A). However, unlike the susceptibility to the antibiotic in $0.3 \mathrm{~g} / \mathrm{L} \mathrm{TSB}$, the biofilms were able to recover from the $4000 \mathrm{mg} / \mathrm{L}$ streptomycin exposure even on the early steady state biofilm. Furthermore, a second exposure to the same concentration of streptomycin resulted in an increase in biofilm metabolism that was maintained throughout the 4-h exposure. When the biofilms grown in low citrate $(0.14 \mathrm{mM})$ concentration medium were exposed to antibiotic medium supplemented with $1 \mathrm{mM}$ citrate, $\mathrm{CO}_{2}$ production rapidly increased and remained above steady state values for the duration of the antibiotic exposure. Following the resumption of antibiotic-free growth medium after the first antibiotic exposure, there was a drop in biofilm metabolism below steady state 
levels that rapidly recovered, though subsequent exposures returned to steady state metabolism immediately following the removal of the antibiotic medium (Fig. 2.2B).

\subsubsection{Biofilm metabolic response while switching carbon sources with and without antibiotic present}

While biofilms grown from the start in defined medium with citrate as carbon source were resistant to high streptomycin concentrations, added citrate could not rescue TSB grown biofilms from streptomycin. To explore citrate's inability to rescue biofilms exposed to streptomycin in TSB (glucose containing) growth medium (Fig. 2.1A4), biofilm metabolic changes were delineated when switching from citrate to glucose medium with and without antibiotics present in the medium. When biofilms were grown in a defined medium containing $1 \mathrm{mM}$ glucose and exposed to antibiotic in $1 \mathrm{mM}$ citrate, the metabolism dropped by $\sim 80 \%$ at the end of the 4-h exposure, with rapid recovery once switched back to antibiotic free $1 \mathrm{mM}$ glucose medium (Fig. 2.3A). When biofilms were grown in $1 \mathrm{mM}$ citrate medium and exposed to the antibiotic in 1 $\mathrm{mM}$ glucose medium there was a similar initial drop in metabolism when the carbon source was switched. Conversely, the metabolism recovered before the end of exposure, while further exposures resulted in an increase in metabolism for the 4-h duration (Fig. 2.3B). To demonstrate the effects of switching the carbon source on biofilm metabolism without any antibiotic present media was switched from either $1 \mathrm{mM}$ citrate or glucose media to a $4 \mathrm{~h} 1 \mathrm{mM}$ glucose or citrate exposure. The $\mathrm{CO}_{2}$ production of biofilms shuts down instantaneously when switching from either glucose medium to citrate and vice versa. Without antibiotic present both glucose and citrate biofilms could start to increase their $\mathrm{CO}_{2}$ output levels again during mid exposure. Interestingly, the return to steady state $\mathrm{CO}_{2}$ output took longer when antibiotic was not present in the media (Fig. 2.3C and D). 

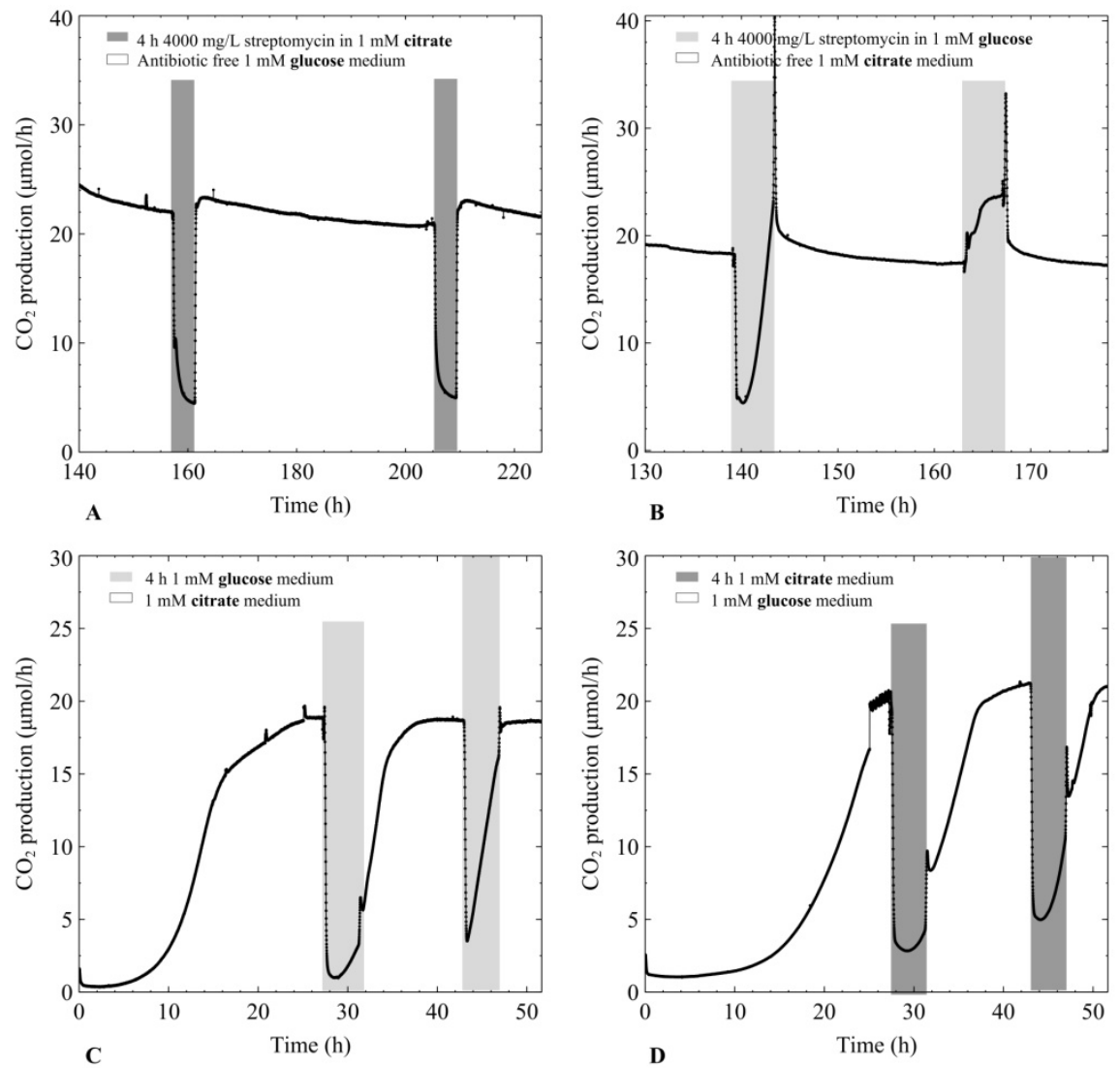

Figure 2.3. The $\mathrm{CO}_{2}$ production $(\mu \mathrm{mol} / \mathrm{h}$ ) of multispecies biofilms grown in defined medium: (A) $1 \mathrm{mM}$ glucose as the carbon source exposed two times to $1 \mathrm{mM} 4000 \mathrm{mg} / \mathrm{L}$ streptomycin in $1 \mathrm{mM}$ citrate, (B) $1 \mathrm{mM}$ citrate exposed two times to $4000 \mathrm{mg} / \mathrm{L}$ streptomycin in $1 \mathrm{mM}$ glucose (C) $1 \mathrm{mM}$ citrate grown biofilms exposed to $1 \mathrm{mM}$ antibiotic free glucose (D) $1 \mathrm{mM}$ glucose grown biofilms exposed to $1 \mathrm{mM}$ antibiotic free citrate.

\subsubsection{Single species biofilm studies to determine the effect of streptomycin on $P$. aeruginosa strain PAO1 and PAO1 $\triangle \mathrm{MexXY}$}

The efflux pump MexXY is a known mechanism of increased resistance to aminoglycoside antibiotics and requires energy to function (Webber and Piddock, 2003; Zhao et al., 1998). For that reason there was interest in determining how a single species biofilm of PAO1 would fair in comparison to a PAO1 $\triangle$ MexXY mutant. It was hypothesized that if the MexXY efflux pump in the multispecies biofilms containing $P$. aeruginosa played a role in the increased resistance to streptomycin antibiotics and was aided when extra carbon was added to the medium, that early steady state biofilms of the $\Delta \mathrm{MexXY}$ mutant would not be aided by additional carbon in the antibiotic medium. Alternatively, it was hypothesized that PAO1 would be aided by additional 
carbon due to the ability of the biofilm to utilize the excess energy towards antibiotic efflux. The results demonstrate that early steady state biofilms of PAO1 were not able to recover from the high dose streptomycin exposure with and without the addition of glucose to the antibiotic medium (Fig. 2.4A1, A2). Similarly, the PAO1 $\triangle \mathrm{MexXY}$ mutant exposed to $4000 \mathrm{mg} / \mathrm{L}$ of streptomycin with and without added carbon was unable to recover from the antibiotic exposure (Fig. 2.4B1, B2). Each of the biofilms, regardless of whether they had the MexXY efflux pump or not, had an initial increase in $\mathrm{CO}_{2}$ production above steady state levels prior to a rapid decrease in $\mathrm{CO}_{2}$ output towards baseline levels, and when additional carbon was added to the antibiotic medium, the initial increase in $\mathrm{CO}_{2}$ production upon antibiotic exposure was greater (Fig. 2.4).
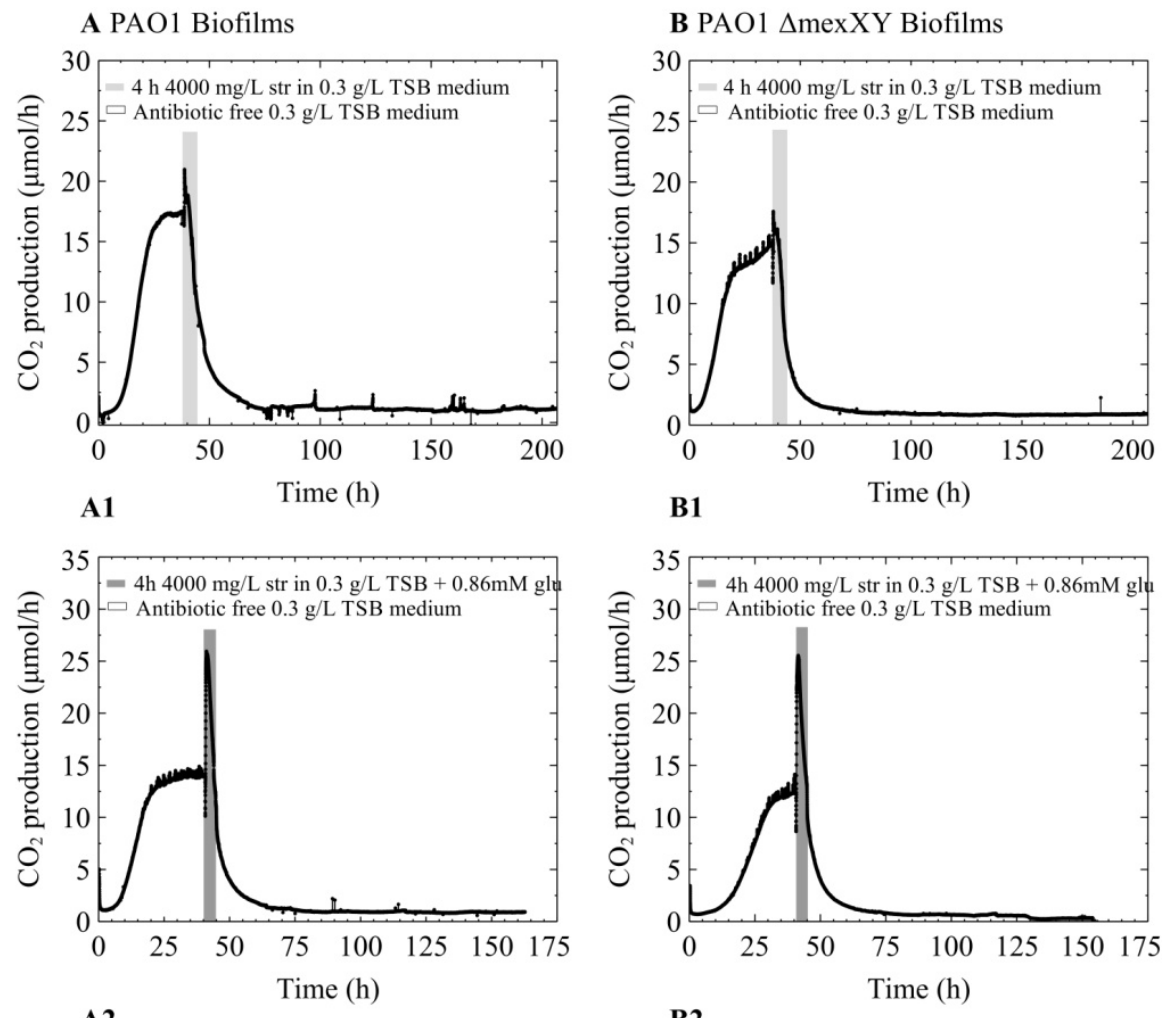

Figure 2.4. $\mathrm{CO}_{2}$ production ( $\mu \mathrm{mol} / \mathrm{h}$ ) of biofilms of $P$. aeruginosa PAO1 (column A) and PAO1 $\triangle \mathrm{MexXY}$ (column B) grown in 0.3 $\mathrm{g} / \mathrm{L}$ TSB medium. The biofilms were grown for less than $48 \mathrm{~h}$ and had reached steady state $\mathrm{CO}_{2}$ output before they were exposed to $4000 \mathrm{mg} / \mathrm{L}$ streptomycin (str) for $4 \mathrm{~h}$. Figures (A1) and (B1) biofilms were exposed to str in $0.3 \mathrm{~g} / \mathrm{L} \mathrm{TSB}$ medium. Figures (A2) and (B2) were exposed to str in $0.3 \mathrm{~g} / \mathrm{L} \mathrm{TSB}$ medium in $0.3 \mathrm{~g} / \mathrm{L} \mathrm{TSB}$ with the addition of $0.86 \mathrm{mM}$ glucose (glu).

Conversely, when the pure culture PAO1 freezer cultures were streak plated prior to growing overnight cultures for biofilm inoculation, the behaviour differed from what is shown in Fig. 2.4A1 and A2. When early steady state biofilms of PAO1 were inoculated from streak plates, as old as two weeks, they were able to recover from the high dose streptomycin exposure with and 
without the addition of carbon to the antibiotic medium (Fig. 2.5A1 and A2). Unlike PAO1, the PAO1 $\triangle$ MexXY strain was unable to recover from high dose streptomycin exposures even when the freezer culture was inoculated onto agar plates prior to growing overnight cultures used for biofilm inoculation (Fig. 2.5B1 and B2).
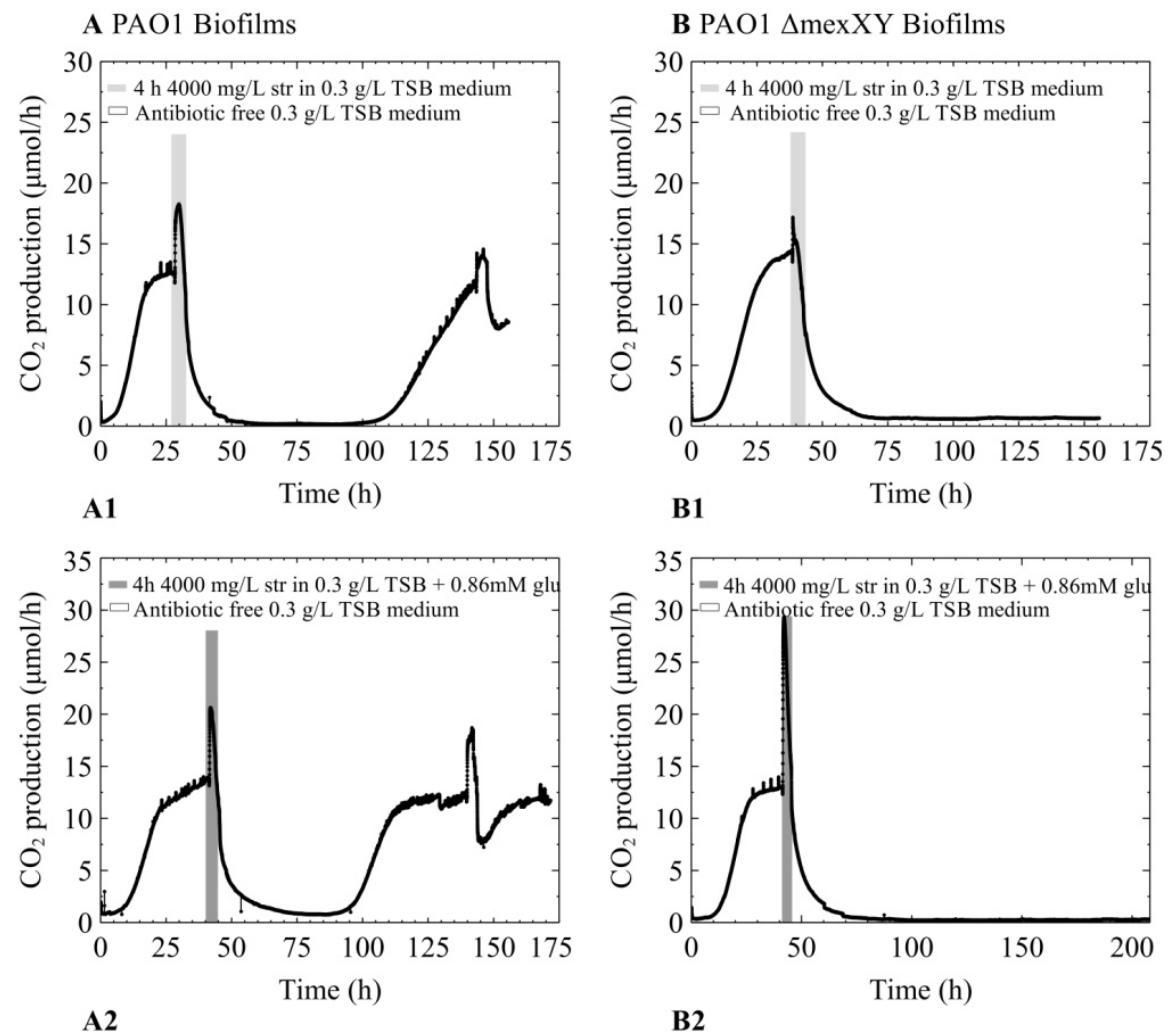

Figure 2.5. The $\mathrm{CO}_{2}$ production ( $\mu \mathrm{mol} / \mathrm{h}$ ) of biofilms of $P$. aeruginosa PAO1 (column A) and PAO1 $\Delta \mathrm{MexXY}$ (column) grown in 0.3 $\mathrm{g} / \mathrm{L}$ TSB medium. The biofilms inoculated from bench cultures were grown for less than $48 \mathrm{~h}$ and had reached steady state $\mathrm{CO}_{2}$ output before they were exposed to $4000 \mathrm{mg} / \mathrm{L}$ streptomycin (str) for $4 \mathrm{~h}$. Figures (A1) and (B1) biofilms were exposed to str in $0.3 \mathrm{~g} / \mathrm{L} \mathrm{TSB}$ medium. Figures (A2) and (B2) were exposed to str in $0.3 \mathrm{~g} / \mathrm{L} \mathrm{TSB}$ medium in $0.3 \mathrm{~g} / \mathrm{L}$ TSB with the addition of 0.86 $\mathrm{mM}$ glucose (glu).

\subsubsection{Adaptation and comparison to other Gram-negative strains}

A culture originating from the same freezer stock culture used in the preceding experiments, but repeatedly subcultured for several years, showed the ability to recover after the first treatment at $4000 \mathrm{mg} / \mathrm{L}$ ( 600x planktonic MIC under similar growth conditions; Fig. 2.6A) and without a drop in metabolism following subsequent exposures, even when the streptomycin concentration was increased to $12000 \mathrm{mg} / \mathrm{L}$ (Fig. 2.6B). The numbers of viable cells released from the biofilms into the effluent reflected the biofilms' response to antibiotic exposure: as the biofilm $\mathrm{CO}_{2}$ production decreased, so did the number of viable cells in the effluent. For example, the effluent cell numbers decreased from $2.0 \times 10^{7}$ at steady state to $1.6 \times 10^{3}$ and $9.0 \times 10^{2} \mathrm{CFU} / \mathrm{ml}$ in the 
effluent mid exposure, and at the end of the first $4 \mathrm{~h}$, respectively. In contrast, the second exposure did not result in a similar decrease in effluent cell number; effluent cell numbers were $2.4 \times 10^{5} \mathrm{CFU} / \mathrm{ml}$ at the end of the $4 \mathrm{~h}$ exposure.

Tests were performed on the three Gram-negative environmental isolates to demonstrate that the effects of high doses of streptomycin on biofilm metabolism were not unique to the multispecies culture biofilms. Biofilms formed by the three environmental strains showed similar responses when exposed to $4000 \mathrm{mg} / \mathrm{L}$ streptomycin in low concentration $(0.3 \mathrm{~g} / \mathrm{L}) \mathrm{TSB}$ medium. In each case, metabolic activity (as indicated by $\mathrm{CO}_{2}$ production) dropped to near-baseline levels, and took between 48 and $120 \mathrm{~h}$ to recover to pre-exposure levels (Fig. 2.6C-E). When subsequently exposed to the antibiotic, the biofilms consistently increased their metabolism as an immediate response to the exposure, typically restoring their steady state metabolic rate to pre-exposure levels within $24 \mathrm{~h}$. When a 5 day old multispecies biofilm (inoculated from freezer stocks) was exposed to $4000 \mathrm{mg} / \mathrm{L}$ streptomycin the metabolic response resembled that of the environmental isolates and bench grown cultures (Fig. 2.6F). Unlike the early steady multispecies biofilms (Fig 2.1A1-A3) the older biofilm could recover following high dose streptomycin exposures.

\subsubsection{Minimum inhibitory concentration of streptomycin in various media types}

The MICs of multispecies cultures as well as the environmental isolates in low and high TSB growth medium ( $0.3 \mathrm{~g} / \mathrm{L}$ and $3 \mathrm{~g} / \mathrm{L}$ respectively) ranged between $0.5-3.5 \mathrm{mg} / \mathrm{L}$. When grown in defined medium with either high or low carbon content ( $1 \mathrm{mM}$ or $0.14 \mathrm{mM}$ citrate or glucose, and $2 \mathrm{mM}$ or $0.28 \mathrm{mM}$ pyruvate) the multispecies culture inoculum was able to grow in higher streptomycin concentrations (from $14 \mathrm{mg} / \mathrm{L}$ up to $50 \mathrm{mg} / \mathrm{L}$ ) compared to when grown in $3 \mathrm{~g} / \mathrm{L}$ TSB growth medium. Previous exposure to streptomycin had an impact on the MIC of the cells released from biofilms. For instance, the streptomycin MIC of the inoculum as well as effluent cells collected from biofilms grown on $0.3 \mathrm{~g} / \mathrm{L}$ TSB was $3.5 \mathrm{mg} / \mathrm{L}$, compared to effluent cells collected following high dose streptomycin exposure, which had an MIC of $200 \mathrm{mg} / \mathrm{L}$ (see asterisks in Fig. 2.6A). 

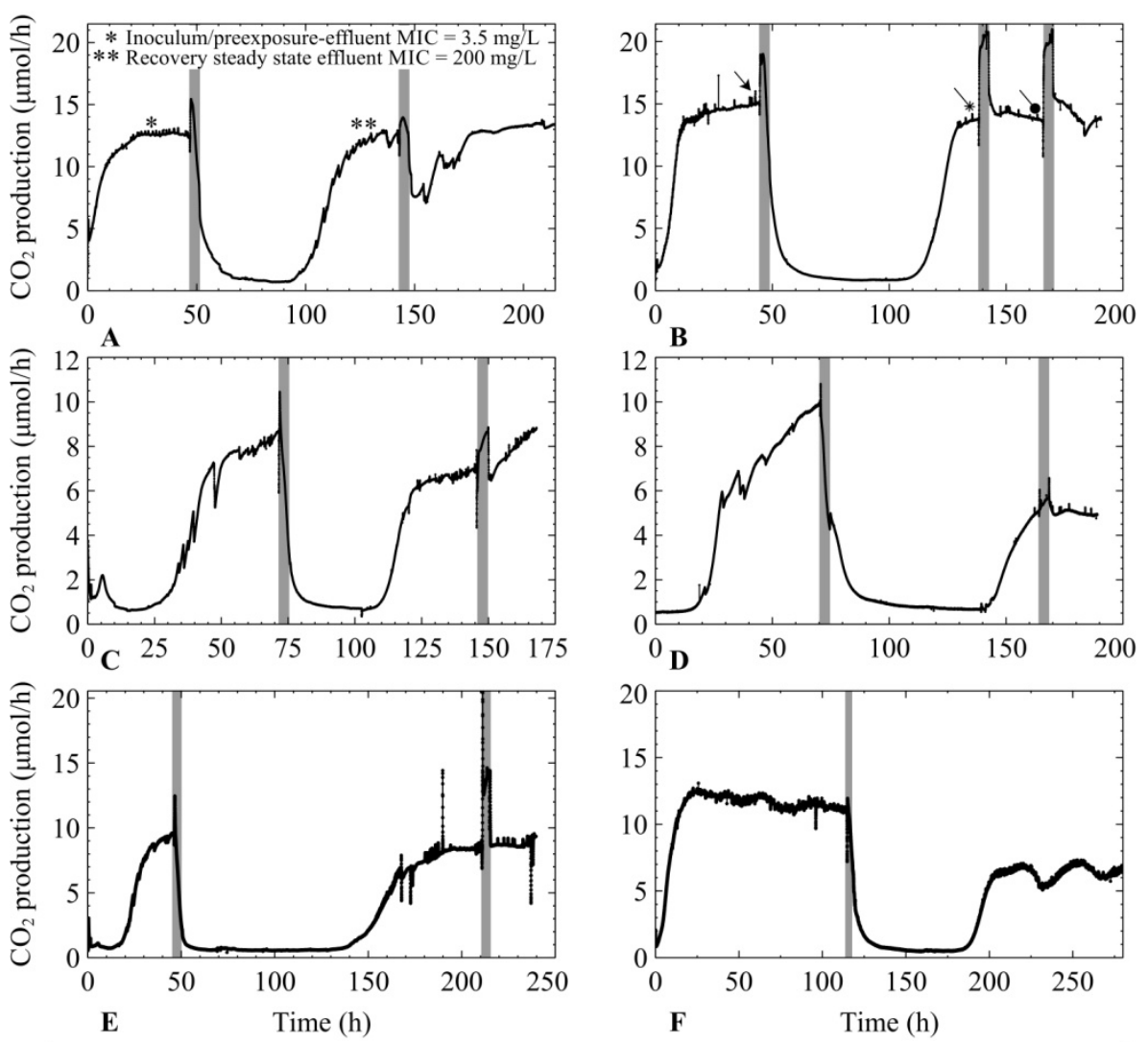

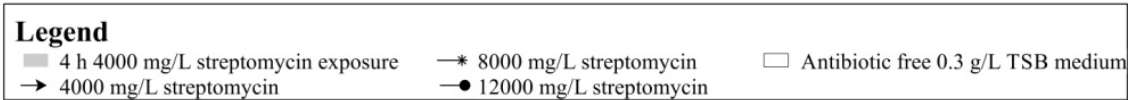

Figure 2.6. The $\mathrm{CO}_{2}$ production $(\mu \mathrm{mol} / \mathrm{h}$ ) of bacterial isolates exposed to streptomycin in $0.3 \mathrm{~g} / \mathrm{L} \mathrm{TSB}$ medium for 4-h intervals. (A) multispecies bench culture grown on $0.3 \mathrm{~g} / \mathrm{L} \mathrm{TSB}$ medium and exposed to $4000 \mathrm{mg} / \mathrm{L}$ streptomycin. The effluent cell numbers decreased from $2.0 \times 10^{7} \mathrm{CFU} / \mathrm{ml}$ at steady state to $9.0 \times 10^{2} \mathrm{CFU} / \mathrm{ml}$ at the end of the first $4 \mathrm{~h}$ exposure. In contrast, the second exposure did not result in a similar decrease of effluent cell numbers; which were $2.4 \times 10^{5} \mathrm{CFU} / \mathrm{ml}$ at the end of the $4 \mathrm{~h}$ exposure (B) $P$. aeruginosa on $0.3 \mathrm{~g} / \mathrm{L} \mathrm{TSB}$ medium and exposed to either 4000,8000 , or $12,000 \mathrm{mg} / \mathrm{L}$ streptomycin (C) an Enterobacter species biofilm (D) Enterobacter asburiae biofilm, (E) a Pseudomonas putida biofilm, and (F) multispecies biofilm grown for 5 days prior to the streptomycin exposure.

\subsection{Discussion}

The data demonstrate that biofilm response to antibiotic exposure is dependent upon the type of medium in which the biofilm is grown, the amount of available carbon during the antibiotic exposure, the carbon source, and the length of time the biofilm is at steady state prior to exposure. Mechanisms responsible for the survival of bacterial biofilms were not tested in this study, and are the focus of an ongoing investigation. Various forms of resistance have been reported in the literature, such as stable resistance and adaptive resistance that are characterized by genetic changes and down regulation of antibiotic uptake, respectively (Fernández and Hancock, 2012). Another way bacteria can survive an antibiotic exposure is through antibiotic 
tolerance. Antibiotic tolerance occurs when bacteria survive an antibiotic exposure through inactivity or dormancy rather than from genetic changes or active resistance mechanisms (Lewis, 2007). Antibiotics, which normally target some form of macromolecular synthesis, have no targets to act on since dormant cells are not growing and are mostly inactive (Rozenkranz, 1964; Cotsonas and $\mathrm{Wu}, 2009$; Lewis, 2007). For the purpose of this discussion the term antibiotic resistance is not used to describe biofilm behavioural changes in response to streptomycin. It has been pointed out that cells within biofilms may not have more intrinsic resistance compared to their planktonic counter parts (Lewis, 2001). The growth state of individual cells cannot be addressed from this study as whole-biofilm response is monitored. Nevertheless, the data presented here shows that carbon content and growth conditions can affect microbial metabolic response to antimicrobials. Using the CEMS for monitoring whole biofilm metabolism was useful to guide experimental design for further testing to delineate antibiotic resistance mechanisms by providing cues for plausible mechanisms of tolerance and resistance. Moreover, the CEMS may provide a better simulation of real-world biofilms that typically show spatial variability.

Pre-steady state and early steady state multispecies inoculum biofilms were susceptible to high concentration streptomycin exposures (Fig. 2.1A1-A3). The graphs from Figure 2.1A1-A3 show biofilms that are under $48 \mathrm{~h}$ old exposed to streptomycin and are susceptible to the antibiotic. This is in contrast to experiments that were performed in which 4-5day old biofilms were less susceptible to streptomycin and recovered from the same $4000 \mathrm{mg} / \mathrm{L}$ streptomycin exposure in $0.3 \mathrm{~g} / \mathrm{L}$ TSB medium (Fig. 2.6F). This suggests that biofilm growth stage or age plays a role in biofilm susceptibility to streptomycin, which is consistent with literature (Ito et al., 2009; Chopra et al., 2015). Using the CEMS the focus is on the metabolic profile of the biofilm; underlying reasons for increased survival of aged biofilms may include increased thickness, population density, changes in gene expression, and inactivation of lower layers of the biofilm (Stewart, 2002; Stewart, 1994), each of which can contribute to increased antibiotic resistance and may be seen on the $\mathrm{CO}_{2}$ profiles as a lower overall effect on whole biofilm metabolism. Increased biofilm thickness may result in higher levels of extracellular polymeric substances being produced such as negatively charged alginate in $P$. aeruginosa that binds to positively charged aminoglycoside antibiotics (Stewart and Costerton, 2001; Gordon et al., 1988). In addition, antimicrobials take longer to diffuse through thicker biofilms allowing more time for biofilms to 
upregulate adaptive stress responses including excretion of antimicrobial degrading enzyme or increasing EPS production (Szomolay et al., 2005). Adaptive stress responses, for example nutrient stressors or antibiotic stressors, can also lead to enhanced ability to survive high concentration antibiotic exposures (de la Fuente-Núñez et al., 2013; Stewart, 2002). Furthermore, as biofilms age their cell density increases and eDNA accumulates (Fux et al., 2005; AllesenHolm et al., 2006). Extracellular DNA can bind to cations such as $\mathrm{Mg}^{2+}$ and $\mathrm{Ca}^{2+}$ that help stabilize LPS molecules in the outer membrane. The binding of eDNA to cations leads to changes in gene expression resulting in an altered outermembrane structure more resistant to cationic antimicrobial peptides and aminoglycoside antibiotics (Mulcahy et al., 2008). Ionic binding to the outer membrane is a crucial stage in aminoglycoside uptake (Hancock, 1981).

Most traditional antibiotics are effective against bacteria during their growth phase since antibiotics affect metabolic processes such as protein synthesis (aminoglycosides), DNA synthesis (fluorquinolones), and cell wall synthesis ( $\beta$-lactams) (Cotsonas and $\mathrm{Wu}, 2009$; Hancock, 1981; Eng et al., 1991; Hooper, 2001). Accordingly, many studies have demonstrated the loss of antibiotic efficacy when cells are in a non-growing or starved state (Nguyen et al., 2011) and that the addition of certain nutrients can re-establish bacterial susceptibility to antibiotics (Boriello et al., 2001; Allison et al., 2011).

Contrasting the notion that the addition of nutrients increases antimicrobial susceptibility, the data demonstrate that excess nutrients provided along with the antibiotic media aided young biofilms in recovering from antibiotic exposures. The initial hypothesis was that additional nutrients would aid biofilm recovery from aminoglycoside addition since an initial spike in metabolism upon antibiotic addition was seen. The results presented here demonstrate that the addition of excess carbon to the antibiotic medium in the form of TSB, glucose, or pyruvate reduced the young biofilms' susceptibility to streptomycin (Fig. 2.1B1-B3) compared to when no additional nutrients were added (Fig. 2.1A1-A3). The spike in metabolism that occurs in the presence of the added-carbon antibiotic media typically occurs in about 15 min compared to an increase in $\mathrm{CO}_{2}$ output of $1 \mathrm{~h}$ at room temperature as a result of bacterial doubling time (in $3 \mathrm{~g} / \mathrm{L}$ TSB medium) (Ronan, 2011). The large and rapid increase in metabolism (specifically Fig. 2.1B1-1B2) when TSB or pyruvate was added to the antibiotic medium suggests that the biofilms were able to utilize the excess nutrients upon exposure, circumventing the detrimental 
effects of the antibiotic. Further testing would need to be performed to test the hypothesis that pyruvate or the nutrients in TSB can be utilized during streptomycin uptake. One way to test nutrient utilization would be to monitor the consumption of glucose and pyruvate before, during and after antibiotic exposure using enzymatic assay kits. Studying how these nutrients impede negative effects of high doses of streptomycin on young biofilms may be more challenging. One would have to learn what physiological changes occur during the uptake of the carbon or nutrients and how these changes are affecting streptomycin uptake. A study performed by Buchholz et al. (2010) monitored biofilm heat-production in real time and found that when biofilms were exposed to antibiotics heat production spiked initially before declining along with ATP levels. Their work supports the hypothesis that metabolism increases during the antibiotic exposure and that excess nutrients can be utilized (Buchholz et al., 2010).

Previous work has shown a relation between carbon addition and changes in antibiotic susceptibility. For example, studies performed on E. coli, whose preferred carbon source is glucose, have demonstrated that additional glucose leads to catabolite repression through repressing cAMP (Dalhoff, 1979) and that glucose addition can impede the antimicrobial effects of streptomycin (Zuroff et al., 2010). Furthermore, the addition of glucose in the antibiotic medium can decrease susceptibility to kanamycin in biofilms of E. coli; though no mechanism was given, the authors alluded to the ability of glucose to repress the uptake of other catabolites leading to antibiotic tolerance (Palmer et al., 2007). A link to nutrient uptake or metabolism and decreased susceptibility to antibiotics in P. aeruginosa is a possibility as well; however, the mechanisms likely differ from those in E. coli. P. aeruginosa does have catabolite repression control, but glucose is not the preferred carbon source. $P$. aeruginosa prefers amino acids and organic acids to glucose (Mukkada et al., 1973; Ng and Dawes, 1967; Collier et al., 1996). The behaviour of the biofilm will also depend on how the carbon sources fed into the biofilm affect other species present. Studies that have considered the behaviour of Stenotrophomonas spp with aminoglycosides in the presence of various carbon sources were not discovered. Presumably, with the limited nutrient profile of S. maltophilia (Stanier et al., 1966) its behaviour in the presence of various nutrients would largely depend on if it could metabolize the given nutrient and/or how the other species present behave. 
Only carbon sources that were readily metabolized by the biofilm enhanced survival to streptomycin; as seen when glucose, pyruvate, or TSB were added (but not citrate) to the antibiotic medium (Fig. 2.1A4; Fig. 2.3A-D). The inability of excess citrate in the medium to enhance survival might be explained by the fact that the TSB grown biofilms were not adapted for citrate utilization (Fig. 2.1A4). TSB is a rich medium that contains glucose as the carbon source. Therefore citrate's inability to rescue a TSB grown biofilm can potentially be explained by catabolite repression, which may occur when glucose and citrate are in the same medium. Figure 2.3A-D demonstrates the concept behind catabolite repression as the biofilms shut down their metabolism when a different carbon source was supplied in the medium. It has been shown that citrate-grown $P$. aeruginosa did not up-regulate glucose metabolizing enzymes until a threshold glucose concentration was reached, yet in glucose medium, citrate addition immediately resulted in the induction of the citrate transport system (Whiting et al., 1976). In this study the glucose and citrate were not administered at the same time thus the metabolism reflects the ability of the biofilm to utilize the carbon source administered at the time. Conversely, with addition of pyruvate to the TSB medium, bacteria can be expected to grow faster than with either a media containing glucose or pyruvate alone ( $\mathrm{Ng}$ and Dawes, 1973).

In addition to the effects of added carbon during streptomycin exposure, the data demonstrate that the type growth medium affects whole-biofilm metabolic response to streptomycin.

Replicate experiments showed that when biofilms were grown in a defined growth medium with low (0.14 mM) carbon concentration (Fig. 2.2A), biofilm susceptibility to streptomycin was diminished compared to when grown in $0.3 \mathrm{~g} / \mathrm{L}$ TSB medium (containing $0.14 \mathrm{mM}$ glucose). Consistent with previous results, addition of carbon to the antibiotic medium reduced the biofilm's susceptibility to streptomycin (Fig. 2.2B). It is plausible that changes in medium composition and carbon content alter the relative abundance of the members in multispecies biofilms and their physiological responses to the antibiotic exposures.

Results of this study indicate that, in addition to the growth stage of biofilms, the origin of the culture plays a role in the metabolic recovery following a streptomycin exposure (Fig. 2.6A, B). Repeated sub-culturing on agar plates might result in differential gene expression and can select for mutants best adapted for growth under laboratory conditions. It is known that decreased sensitivity to antibiotics may develop at high population density and under stressful 
environmental conditions that lead to rapid up-regulation of stress response genes (Mah and O'Toole, 2001). Bench cultures showed adaptation to the antibiotic by increasing their metabolic output throughout the 4-h exposures once preconditioned to the antibiotic. The increased $\mathrm{CO}_{2}$ output indicates that biofilms can actively prevent the antibiotic from disrupting the overall biofilm metabolism. Following a second streptomycin exposure the biofilm metabolism rapidly returned to steady state levels following the resumption of antibiotic free growth medium.

Gram-negative environmental isolates had similar $\mathrm{CO}_{2}$ profiles when exposed to high concentrations of streptomycin in low concentration $(0.3 \mathrm{~g} / \mathrm{L}) \mathrm{TSB}$. Changes in the biofilm metabolic profile of the bacterial isolates, (Fig. 2.6C-E) from the first antibiotic exposure to the second, demonstrate similar patterns in metabolic behaviour. Upon analysis of each of the biofilms' metabolic responses to the antibiotic exposure, it was clear that the biofilms respond initially to high concentrations of streptomycin by increasing their energy output, and if primed for the exposure they will maintain their high metabolic output levels throughout the exposure. Biofilms formed by all of the environmental isolates and the lab cultures in this study were able to survive antibiotic concentrations that were higher than their planktonic MIC. The MIC's for Enterobacter isolates have been reported from $8 \mathrm{mg} / \mathrm{L}$ to as high as $256 \mathrm{mg} / \mathrm{L}$ (Chiew et al., 1998); one study found streptomycin MIC of environmental strains of $P$. putida to be $>512$ $\mathrm{mg} / \mathrm{L}$ but considered levels $>16 \mathrm{mg} / \mathrm{L}$ to be the resistance breakpoint for streptomycin (Bezanson et al., 2008); environmental isolates of S. maltophilia showed streptomycin MIC's from $64 \mathrm{mg} / \mathrm{L}$ even without any resistance genes detected (Popowska et al., 2012); finally, another study demonstrated the streptomycin MIC for P. aeruginosa was $64 \mathrm{mg} / \mathrm{L}$ in Mueller-Hinton Broth (Morita et al., 2001).

In an attempt to further explore a potential mechanism of antibiotic resistance and/or a reason for survival of young biofilms following the addition of excess carbon to the media, single species biofilm of PAO1 and a PAO1 $\triangle \mathrm{MexXY}$ mutant deficient in the ability to efflux streptomycin antibiotics were tested. The initial hypothesis that the increased $\mathrm{CO}_{2}$ output may be linked to an energy induced antibiotic resistance mechanism was not supported based on the freezer culture inoculum. The results cannot clearly indicate that the MexXY efflux pump plays a role in survival to the antibiotic as seen in Figure 2.4, since neither PAO1 nor PAO1 $\triangle \mathrm{MexXY}$ strain could recover from the antibiotic exposures even with the addition of carbon to the antibiotic 
media. At this point it is not clear why the early steady state single species biofilm of PAO1 would not be aided by the addition of glucose to the antibiotic media, while the multispecies biofilms were. Interestingly, when biofilms of pure PAO1 and $\triangle \mathrm{MexXY}$ strains were inoculated from bench cultures the PAO1 strain had the ability to survive the antibiotic exposures while the PAO1 $\triangle$ MexXY strain did not, even without the addition of carbon (Fig. 2.5). Therefore, there may be a link to the efflux pumps for the pure cultures in particular. As for the multispecies cultures, there seems to be a link to energy requiring resistance mechanisms that can be initiated by added energy in the form of a readily useable carbon sources. This concept will need to be further explored in relation to the multispecies cultures since P. aeruginosa and S. maltophilia have different preference for carbon sources. The difference in carbon source preferences could explain why the freezer cultures from multispecies biofilms recovered upon glucose addition but the pure PAO1 cultures inoculated from freezer stocks did not. As previously stated, $P$. aeruginosa prefers organic acids to glucose (Ng and Dawes, 1967). Most naturally-occurring biofilms are formed by multispecies communities. The ways in which the species in a multispecies community interact will determine biofilm physiology, structure, and behaviour (Yang et al., 2001). Considering the growing evidence of multispecies infections, studies aimed at delineating interactions between members of biofilm communities during exposure to antibiotics merit recognition in future studies. 


\section{Chapter 3}

\section{The influence of $\mathrm{pH}$ on whole-biofilm susceptibility to gentamicin and streptomycin as monitored by $\mathrm{CO}_{2}$ production}

*This chapter has been submitted for publication as: Jackson, L., Kroukamp, O., Ronan, E. and Wolfaardt, G.M., The influence of $\mathrm{pH}$ whole-biofilm susceptibility to gentamicin and streptomycin as monitored by $\mathrm{CO}_{2}$ production, 2015 (Writing was completed in consultation with the co-authors.) 


\section{The influence of $\mathrm{pH}$ on whole-biofilm susceptibility to gentamicin and streptomycin as monitored by $\mathrm{CO}_{2}$ production}

\subsection{Abstract}

Aminoglycoside antibiotics require a driving force to cross the bacterial membrane. The negative electrical potential in the interior membrane can initiate bacterial uptake of positively charged aminoglycosides at physiological $\mathrm{pH}$. Changes in $\mathrm{pH}$ cause shifts in electrical potential in order to maintain the proton motive force, which can affect aminoglycoside uptake. Previous studies on planktonic cultures have shown that aminoglycoside uptake and killing is reduced at acidic $\mathrm{pH}$ compared to basic $\mathrm{pH}$. Similar studies have not been performed on biofilms containing clinically relevant opportunistic pathogens. Therefore, this study asked the question whether biofilm susceptibility to high concentrations of aminoglycoside antibiotics could be perturbed or enhanced by changing external $\mathrm{pH}$. To test whole-biofilm susceptibility to aminoglycoside antibiotics, a $\mathrm{CO}_{2}$ evolution measurement system (CEMS) was used to delineate the metabolism of multispecies biofilms (containing S. maltophilia and P. aeruginosa) during aminoglycoside exposures at $\mathrm{pH}$ 6.0, 7.0, and 7.4. At steady state, in unbuffered TSB medium, the biofilm effluent $\mathrm{pH}$ was close to neutral. For the antibiotic exposures, biofilms were grown in $0.3 \mathrm{~g} / \mathrm{L}$ TSB medium for $24 \mathrm{~h}$ prior to being exposed to $4000 \mathrm{mg} / \mathrm{L}$ of streptomycin or gentamicin for 4 h. Following the exposures, biofilms were given 7 days to recover in antibiotic-free growth medium. Contrasting to previous planktonic results with aminoglycosides antibiotic, exposures at the acidic $\mathrm{pH}$ of 6.0 resulted in increased susceptibility of the biofilms to gentamicin compared a $\mathrm{pH}$ of 7.0 or 7.4. Biofilms were susceptible to streptomycin at both acidic and basic $\mathrm{pH}$ of 6.0 and 7.4, respectively. However, biofilms could recover from streptomycin exposures at neutral $\mathrm{pH}$ 7.0. The biofilms tested in this study did not show the expected trends in susceptibility to aminoglycoside antibiotics at the $\mathrm{pH}$ tested. Increased survival of the biofilm at a neutral $\mathrm{pH}$ of 7.0 may reflect the biofilms preference for neutral $\mathrm{pH}$ and ability to overcome toxic antibiotic exposures in this state.

\subsection{Introduction}

Biofilms adhered to medical devices, open wounds, and mucus membranes on patients are a main cause of life threatening infections contracted while in hospital (Lindsay and von Holy, 2006; Costerton et al., 1999). Far too often antibiotics are ineffective at treating biofilm 
infections which may exhibit high tolerance to antibiotics effective at treating their planktonic counterparts. Aminoglycoside antibiotics are used in the treatment of a variety of infections such as: septicaemia, urinary tract infections, ostitis, life-threatening Gram-negative infections etc (Gonzalez III and Spencer, 1998). Aminoglycosides, specifically tobramycin and gentamicin, are clinically relevant antibiotics used in the treatment of biofilm infections and are highly effective at treating susceptible P. aeruginosa (Hoffman et al., 2005; Geller, 2009). The intrinsic resistance of bacteria to aminoglycosides and reasons for biofilm tolerance have been extensively studied (Moore and Flaws, 2011; Lambert, 2002; Stewart, 2002; Lewis, 2001). In addition to studies on mechanisms of resistance and tolerance to aminoglycoside antibiotics, research on aminoglycoside uptake and mode of action have allowed many researchers to discover ways to manipulate environmental conditions that will enhance or diminish antibiotic susceptibility (Hancock et al., 1981; Fraimow et al., 1991; Eisenberg et al., 1984).

Understanding bacterial physiology and the properties of aminoglycosides has helped design more successful in vitro antibiotic treatments. The inner cytoplasmic membrane of bacterial cells is electrochemically negative with respect to the outside of the cells and averages $-100 \mathrm{mV}$ or greater in magnitude (Shapiro, 2000). The membrane potential in bacterial cells is comprised of electrical and chemical components that are utilized for the uptake and excretion of substances. The membrane potential is required for physiological processes, including ATP synthesis, flagellar movement, and growth (Eisenberg, 1984; Busalmen, 2005). Aminoglycoside antibiotics, which target protein synthesis, are cationic at physiological $\mathrm{pH}$ and can utilize the negative electrical potential as a driving force for entry into bacterial cells (Taber 1987; Damper and Epstein, 1981). For example, entry of the aminoglycoside streptomycin into bacterial cells was first shown to require a functional electron transport chain by Hancock (1962) since electron transport chain inhibitors prevented streptomycin uptake and subsequent lethality. In addition, a study performed by Mates et al. (1982) demonstrated that a threshold negative electrical potential is required to drive aminoglycosides across the bacterial membrane and initiate bacterial killing. However, experiments performed on E. coli grown in the presence of CCCP (an electrical potential inhibitor) and sequentially exposed to the aminoglycoside tobramycin, indicated that aminoglycosides can cause killing even in the absence of electrical potential. Aminoglycoside uptake is thus thought to be an energy dependent process requiring either the electrical potential or ATP production (Fraimow, 1991). Consequently, lack of aminoglycoside entry into the cell 
can occur in low energy states or when there is a lack of electrochemical gradient. Having a clear understanding of the link between energy and aminoglycoside efficacy has important therapeutic consequences, and thus it has been studied fairly extensively.

Previous studies have demonstrated a link between $\mathrm{pH}$ and the electrical potential across the bacterial membrane (Damper and Epstein, 1981; Whooley and McLoughlin, 1983), which, in turn, influences aminoglycoside uptake and killing. Two components make up the proton motive force: the electrical potential and the chemical proton potential (Breeuwer and Abee, 2004). The electrical and chemical proton potentials are closely linked in order to maintain the pmf. When the external $\mathrm{pH}$ is equal to the internal $\mathrm{pH}$, the proton motive force is largely made up of the electrical potential. As the external $\mathrm{pH}$ becomes more acidic compared to the interior there is a larger difference in $\mathrm{pH}$ across the membrane and the electrical difference across the membrane subsequently drops (Damper and Epstein, 1981). As an example, one study found the electrical potential across the membrane to be $-85 \mathrm{mV}$ at pH 5.0 and $-142 \mathrm{mV}$ at $\mathrm{pH} 8.0$ (Felle et al., 1980). Acidic $\mathrm{pH}$ have been shown to decrease aminoglycoside efficacy where as more basic $\mathrm{pH}$ will enhance aminoglycoside efficacy in planktonic systems, presumably due to the more negative electrical potential interiorly (Xiong et al., 1996). Thus there was interest in determining how $\mathrm{pH}$ might affect biofilm susceptibility to aminoglycosides. Biofilms have been shown to withstand antibiotic concentrations up to 1000 times greater than their planktonic minimum inhibitory concentrations (Mah and O'Toole, 2001), thus understanding how changes in $\mathrm{pH}$ alter susceptibility to these high antibiotic concentrations could help guide antibiotic treatments. It was hypothesized that biofilm survival to high concentration gentamicin and streptomycin exposures would be $\mathrm{pH}$ dependent and that antibiotic media at $\mathrm{pH} 6.0$ and 7.0 would result in increased biofilm survival compared to antibiotic media at $\mathrm{pH}$ 7.4.

The main focus of this study was to look at how $\mathrm{pH}$ can influence the effects of high doses of aminoglycosides on whole-biofilm metabolic susceptibility and subsequent recovery. 


\subsection{Materials and Methods}

\subsubsection{Bacterial strains and culture conditions}

A multispecies culture was used for all antibiotic susceptibility experiments. Metagenomic analysis indicated that the two dominant species within the multispecies community were $P$. aeruginosa and Stenotrophomonas maltophilia. Freezer stocks of the multispecies culture were maintained in $20 \%$ glycerol at $-20{ }^{\circ} \mathrm{C}$. Biofilm precultures were grown in $3 \mathrm{~g} / \mathrm{L}$ tryptic soy broth (TSB) at $37{ }^{\circ} \mathrm{C}$, with agitation at $300 \mathrm{rpm}$, for $18-24 \mathrm{~h}$ prior to biofilm inoculation.

\subsubsection{Biofilm Development}

Biofilms were grown aerobically in a $\mathrm{CO}_{2}$ evolution measurement system (CEMS), which is a continuous flow system that enables real-time monitoring of whole biofilm metabolism (Kroukamp and Wolfaardt, 2009). The CEMS is comprised of an inner silicone tube that is permeable to gas within an impermeable outer tube. A continuous supply of growth medium is fed into the inner silicone tubing where biofilm attachment and growth takes place. The residence time of fluid in the CEMS is $8 \mathrm{~min}$. Therefore planktonic cell metabolism did not contribute significantly to $\mathrm{CO}_{2}$ production as the flow rate exceeded planktonic growth rate by at least 10 times. The gas permeable silicone tubing enables the $\mathrm{CO}_{2}$ produced by the biofilm to be collected by a $\mathrm{CO}_{2}$-free sweeper gas (air, TOC, Linde Industrial Gases, ON, Canada) and measured in real-time in a $\mathrm{CO}_{2}$ analyzer (Kroukamp and Wolfaardt, 2009). The medium used for biofilm growth and antibiotic exposures was fed into the CEMS using a peristaltic pump (at a flow rate of $15 \mathrm{ml} / \mathrm{h}$ in this study). The CEMS apparatus was immersed in a water bath to keep a constant temperature of $25^{\circ} \mathrm{C}$.

\subsubsection{Measuring effluent $\mathrm{pH}$ following $\mathrm{pH}$ adjustments on growth medium fed to steady state biofilms}

The biofilm grown in $0.3 \mathrm{~g} / \mathrm{L} \mathrm{TSB}$ at $\mathrm{pH} 7$ was allowed to develop in the CEMS until the production of $\mathrm{CO}_{2}$ stabilized. Once metabolically stable, the growth medium $\mathrm{pH}$ was adjusted, using either $1 \mathrm{M} \mathrm{NaOH}$ or $1 \mathrm{M} \mathrm{HCl}$ drop-wise, to $\mathrm{pH} 5.0, \mathrm{pH} 6.0$, or 8.0. The $\mathrm{pH}$ of the biofilm effluent was measured after $1 \mathrm{~h}$ to allow the medium to reach the biofilm and replace several reactor volumes. 


\subsubsection{Biofilm antibiotic exposures}

The antibiotic medium was introduced to the biofilms once steady state levels of $\mathrm{CO}_{2}$ were reached; indicated by a stable production of $\mathrm{CO}_{2}$. Either streptomycin sulfate (Biobasic Inc. Markham, ON, CA) or gentamicin sulfate was added directly to sterile growth medium at one of three $\mathrm{pH}: 6.0,7.0$, or 7.4. The $\mathrm{pH}$ of the medium was adjusted using either $1 \mathrm{M} \mathrm{NaOH}$ or $1 \mathrm{M}$ $\mathrm{HCl}$ added drop-wise. Antibiotic exposures were carried out at a concentration of $4000 \mathrm{mg} / \mathrm{L}$ and lasted a total of $4 \mathrm{~h}$; the physiological half-life of streptomycin in the plasma is 2-4 h (SigmaAldrich, St. Louis, Missouri, USA).

\subsubsection{Antibiotic susceptibility testing}

The antibiotic minimum inhibitory concentration (MIC) for multispecies culture was tested in broth cultures using a two-fold serial dilution range in $3 \mathrm{~g} / \mathrm{L}$ TSB (Andrews, 2001). Stock solutions of streptomycin sulfate and gentamicin sulfate (Bio Basic Inc. Markham, ON, CA) were prepared by dissolving the antibiotic powder in sterile distilled water to a final concentration of $10,000 \mathrm{mg} / \mathrm{L}$. The antibiotic concentrations tested ranged from $0.75-6 \mathrm{mg} / \mathrm{L}$ of gentamicin, and from $1.75-300 \mathrm{mg} / \mathrm{L}$ streptomycin. Antibiotic dilutions were added to sterile $15 \mathrm{ml}$ borosilicate test tubes, with the addition of $100 \mu \mathrm{l}$ of a prepared overnight culture or steady state biofilm effluent, and incubated at $37^{\circ} \mathrm{C}$ for $18 \mathrm{~h}$. MIC testing of streptomycin and gentamicin was performed on all precultures and steady state biofilm effluent to monitor the development of resistance. In addition, the steady state effluent was collected before and after exposure of biofilms to antibiotics to determine biofilm effluent antibiotic susceptibility.

\subsubsection{Biofilm effluent viability testing}

To determine the number of viable planktonic cells being released from the multispecies biofilms, $1 \mathrm{ml}$ of biofilm effluent was collected from the CEMS before, during, and after antibiotic exposure. Serial dilution of the effluent were plated on $3 \mathrm{~g} / \mathrm{L}$ TSA and incubated at 37 ${ }^{\circ} \mathrm{C}$. Biofilm effluent samples collected when antibiotics were present in the growth medium were washed twice via centrifugation at $12,000 \mathrm{rcf}$ for 150 seconds and re-suspended in $0.9 \%$ saline solution. The amount of effluent cells lost due to the centrifugation step was no more than an order of magnitude. 


\subsubsection{Flow cell experiments to study effect on biofilm structure}

Multiple channel flow cells with individual channel dimensions of $30 \mathrm{~mm}$ by $4 \mathrm{~mm}$ by $3 \mathrm{~mm}$ (length, width and depth respectively) were milled from Plexiglas, as previously described (Wolfaardt, 1994). A glass coverslip used to seal the flow cells acted as an attachment site for growing biofilm and allowed for microscopic analysis (Bester, 2010).

Flow cells were inoculated with $0.3 \mathrm{ml}$ of overnight culture and no media flow for $30 \mathrm{~min}$ to allow bacterial attachment. Flow of $0.3 \mathrm{~g} / \mathrm{L}$ growth medium $(15 \mathrm{ml} / \mathrm{h})$ was resumed for $24 \mathrm{~h}$ prior to initiating a $4 \mathrm{~h}$ of $4000 \mathrm{mg} / \mathrm{L}$ gentamicin exposure in $0.3 \mathrm{~g} / \mathrm{L}$ TSB medium at either $\mathrm{pH} 6.0$ or $\mathrm{pH}$ 7.4. Antibiotic free growth medium was then fed into the flow cells for either 24 or $120 \mathrm{~h}$ prior to application of live dead staining and microscopy experiments. All treatments were performed in triplicates on different days due to time limitations for microscopic analysis. However the three biofilms grown and exposed under identical conditions were considered as triplicates when performing further analyses. Control experiments were performed in which duplicate biofilms were exposed to antibiotic-free $\mathrm{pH} 6.0$ or $\mathrm{pH} 7.4$ TSB medium for $4 \mathrm{~h}$ then grown for $168 \mathrm{~h}$ after exposure to determine the nature of the biofilms without any antibiotic present. The flow cells were kept in a hybridization oven to keep the temperature stable at $25^{\circ} \mathrm{C}$.

To assess biofilm structure, live/dead staining was applied by stopping the medium flow and injecting flow cells with a $\sim 50 \mu 1$ mixture of the nucleic acid stain Syto $9(15 \mu \mathrm{M})$ and propidium iodide $(30 \mu \mathrm{M})$ (Invitrogen Baclight kit). After 20 min of staining under static conditions, flow of growth medium resumed allowing excess stain to wash away for $15 \mathrm{~min}$ prior to microscopy. Confocal laser scanning microscopy (CLSM) was performed using a Nikon Eclipse 80i-C1 microscope (Nikon Instruments Inc., Canada) on biofilms in 3 separate flow cells for each treatment with a 60x/1.4 NA oil immersion Plan Fluor objective. Ten z-stack images were captured along each flow cell channel. The $488 \mathrm{~nm}$ laser and 515/30 nm detector was used to capture Syto 9 stained cells. Propidium iodide stained cells were excited with the $543 \mathrm{~nm}$ laser and emission was detected with the 650 LP filter. To prevent any Syto 9 emission being detected with the $650 \mathrm{LP}$, the excitation lasers and detection filters were turned on separately using the frame lambda feature in the EZ-C1 image acquisition software package (Nikon Instruments Inc., Canada). Image processing was performed in the NIS elements software (Nikon Instruments Inc., Canada), followed by image conversion into grey scale tif files using Image J (Abramoff et 
al., 2004). Biomass measurements were then carried out using COMSTAT (written in MATLAB v8.1) (Heydorn et al., 2000).

\subsubsection{Metagenomic analysis}

For the purpose of DNA extraction the planktonic cultures were grown in $3 \mathrm{~g} / \mathrm{L}$ TSB medium, while biofilms were grown in $0.3 \mathrm{~g} / \mathrm{L} \mathrm{TSB}$ medium. The genomic DNA of our planktonic culture, whole-biofilm, and biofilm effluent collected from pre-exposed steady state biofilms was extracted using a MoBio UltraClean ${ }^{\circledR}$ Microbial DNA Extraction Kit (MoBio Laboratories INC., Carlsbad, CA, USA) following the manufacturer's protocol. Once extracted, the microbial DNA was kept at $-20^{\circ} \mathrm{C}$ until further testing. The genomic DNA was submitted to The University of Toronto Center for the Analysis of Genome Evolution and Function (CAGEF) for genome sequencing. CAGEF used the MiSeq Personal Sequencer v2 for the whole genome sequencing. Once they completed the genome sequencing we were provided with .FASTA files that were uploaded to mg-RAST server (v3.2) which provides annotation, analyses, and metadata on the uploaded metagenomes.

\subsection{Results}

\subsubsection{Biofilm adjustment of local environment in response to $\mathbf{p H}$ shifts}

The effects of $\mathrm{pH}$ adjusted TSB medium on steady state biofilm metabolism was monitored prior to commencing experiments with antibiotics. Biofilms fed with $0.3 \mathrm{~g} / \mathrm{L}$ TSB medium at $\mathrm{pH}$ 7.0 produced effluent with a $\mathrm{pH}$ of 7.10 . When the same biofilm was fed with $0.3 \mathrm{~g} / \mathrm{L}$ TSB media at $\mathrm{pH} 5.0,6.0$, and 8.0 the $\mathrm{pH}$ of the effluent collected from then CEMS was $\mathrm{pH}$ 6.68, 6.95, and 6.95 respectively, which demonstrates the biofilms ability to shift the $\mathrm{pH}$ of their environment or the medium.

\subsubsection{Antibiotic exposures on biofilms at various $\mathbf{p H}$}

Biofilms were grown until their $\mathrm{CO}_{2}$ production levels stabilized, referred to here as steady state. In Figures 3.1, 3.2, and 3.3 columns A and B represent replicates of the antibiotic exposures at each $\mathrm{pH}$. At $\mathrm{pH} 6$ the biofilms did not recover from either the streptomycin or gentamicin antibiotic exposures even when fed with antibiotic free growth medium for up to 7 days (Fig $3.1)$. 
A. pH 6.0 Gentamicin Exposures

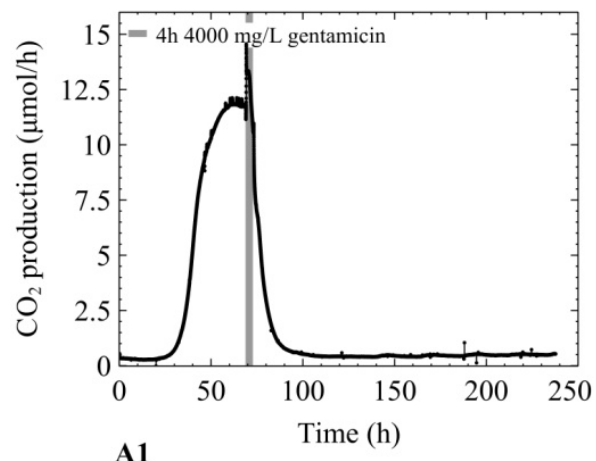

A1

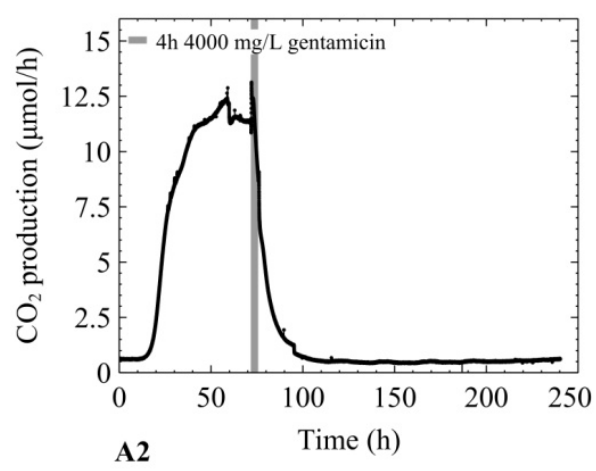

B. pH 6.0 Streptomycin Exposures

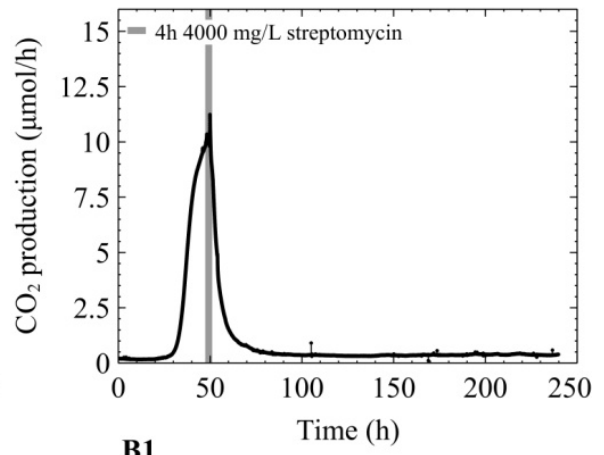

B1

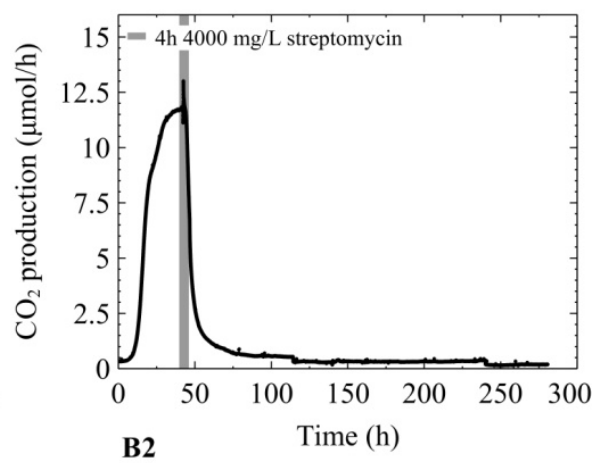

Figure 3.1 Multispecies biofilms were grown in $0.3 \mathrm{~g} / \mathrm{L} \mathrm{TSB}$ medium until a steady state $\mathrm{CO}_{2}$ output was reached prior to being exposed for $4 \mathrm{~h}$ to $4000 \mathrm{mg} / \mathrm{L}$ of either gentamicin (Column A: (A1 and A2)) or streptomycin (Column B: (B1 and B2)) at pH 6.0.

Conversely, when biofilms were exposed to streptomycin and gentamicin at $\mathrm{pH} 7.0$, the biofilms were able to recover from the antibiotic exposures, though the behavioural response to the respective antibiotics differed. The biofilms appeared to adapt to subsequent streptomycin exposures. Biofilm metabolism would drop at or near baseline levels following the first antibiotic exposure, but showed a decreased period for recovery back to steady state for the second exposure (Fig. 3.2B1-2B2). When biofilms were exposed to $4000 \mathrm{mg} / \mathrm{L}$ of gentamicin at $\mathrm{pH} 7$ the biofilms were able to recover back to preexposure steady state $\mathrm{CO}_{2}$ output (Fig. 3.2A1A2). Though, subsequent gentamicin exposures did not always result in recovery (present, but not shown on Fig. 3.2A1; see Fig. 3.4A).

When the biofilms were exposed to $4000 \mathrm{mg} / \mathrm{L}$ gentamicin at $\mathrm{pH} 7.4$ the biofilm recovered from the antibiotic exposure within 7 days (Fig. 3.3A1-A2). Subsequent high dose gentamicin exposures each resulted in a rapid decrease in metabolism towards baseline levels, prior to a recovery period back to steady state levels. Conversely, the biofilms exposed to streptomycin at 
pH 7.4 did not recover even after 7 days of being fed with antibiotic free growth medium (Fig. 3.3B1-B2).

A. $\mathrm{pH} 7$ Gentamicin Exposures

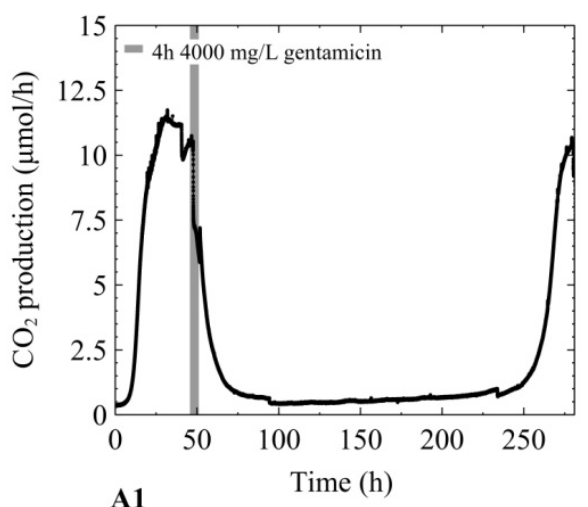

A1

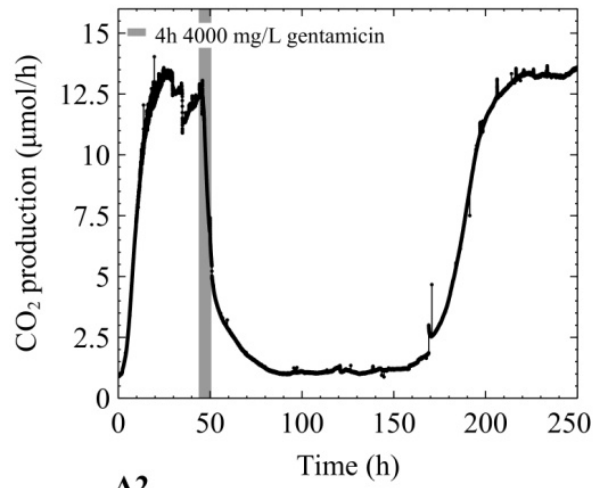

A2
B. $\mathrm{pH} 7$ Streptomycin Exposures

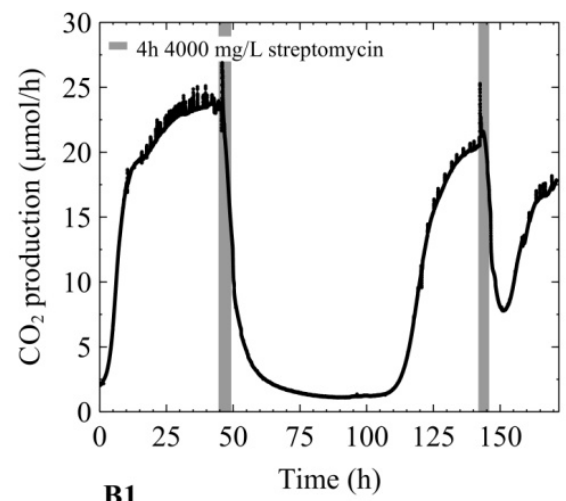

B1

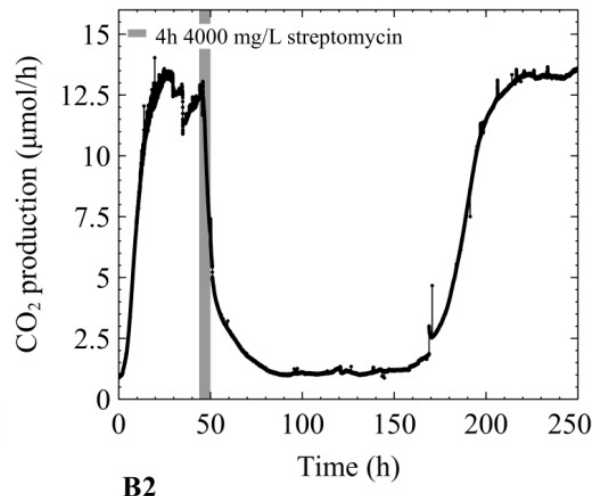

Figure 3.2. Multispecies biofilms were grown in $0.3 \mathrm{~g} / \mathrm{L}$ TSB medium until a steady state $\mathrm{CO}_{2}$ output was reached prior to being exposed for $4 \mathrm{~h}$ to $4000 \mathrm{mg} / \mathrm{L}$ of either gentamicin ((Column A: (A1 and A2)) or streptomycin (Column B: (B1 and B2)) at pH 7.0.

\subsubsection{Biofilm effluent MIC values before and after exposures to high dose streptomycin and gentamicin.}

The overnight cultures used to inoculate biofilms had planktonic MIC's of $3.5 \mathrm{mg} / \mathrm{L}$ and 1.5 $\mathrm{mg} / \mathrm{L}$ for streptomycin and gentamicin, respectively (Table 3.1). Prior to antibiotic exposure, effluent collected from steady state biofilms showed MICs similar to those of the inoculum. Biofilms exposed to either gentamicin or streptomycin at $\mathrm{pH} 6$ and streptomycin at $\mathrm{pH} 7.4$ did not recover from the initial exposures (Fig 3.1 and Fig 3.3B1-B2), thus effluent samples were only obtained prior to the exposure. However, biofilms exposed to streptomycin or gentamicin at pH 7.0 and gentamicin at $\mathrm{pH} 7.4$ did recover following multiple exposures, thus several steady state effluent samples were collected and their MICs tested. When biofilms were exposed to streptomycin at pH 7.0 and recovered (Fig 3.2B1-B2), the biofilm planktonic MIC's increased following recovery after each exposure. Conversely, even when biofilms were exposed to 
gentamicin and able to recover, at $\mathrm{pH} 7.0$ and $\mathrm{pH} 7.4$ (Fig 3.2A1-A2 and Fig 3.3A1-A2), the MICs performed on the biofilm effluent from these samples never increased; remaining at the same level as the initial inoculum at $1.5 \mathrm{mg} / \mathrm{L}$ (Table 3.1 ).

\begin{tabular}{|c|c|c|c|c|c|c|c|c|}
\hline & \multicolumn{8}{|c|}{ MIC Results (mg/L) } \\
\hline & & Effluent pH & \multicolumn{3}{|c|}{ Effluent pH 7.0 } & \multicolumn{3}{|c|}{ Effluent pH 7.4} \\
\hline Antibiotic & $\begin{array}{l}\text { Biofilm } \\
\text { Inoculum }\end{array}$ & $\begin{array}{l}\text { prior to } 1^{\text {st }} \\
\text { exposure }\end{array}$ & $\begin{array}{l}\text { prior to } 1^{\text {st }} \\
\text { exposure }\end{array}$ & $\begin{array}{l}\text { prior to } 2^{\text {nd }} \\
\text { exposure }\end{array}$ & $\begin{array}{l}\text { prior to } 3^{\text {rd }} \\
\text { exposure }\end{array}$ & $\begin{array}{l}\text { prior to } 1^{\text {st }} \\
\text { exposure }\end{array}$ & $\begin{array}{l}\text { prior to } 2^{\text {nd }} \\
\text { exposure }\end{array}$ & $\begin{array}{l}\text { prior to } 3^{\text {rd }} \\
\text { exposure }\end{array}$ \\
\hline Str & 3.5 & 3.5 & 3.5 & 50 & 200 & 3.5 & $\begin{array}{c}\text { No } \\
\text { recovery }\end{array}$ & $\begin{array}{c}\text { No } \\
\text { recovery }\end{array}$ \\
\hline Gm & 1.5 & 1.5 & 1.5 & 1.5 & 1.5 & 1.5 & 1.5 & 1.5 \\
\hline
\end{tabular}

Table 3.1 Gentamicin (Gm) and streptomycin (Str) MIC's of multispecies overnight cultures and steady state biofilm effluent before and after antibiotic exposures. Effluent collected from biofilms that did not recover from the antibiotic exposures did not produce viable cells, thus could not be tested for their planktonic MIC (in the chart labeled as "No recovery").

A. pH 7.4 Gentamicin Exposures

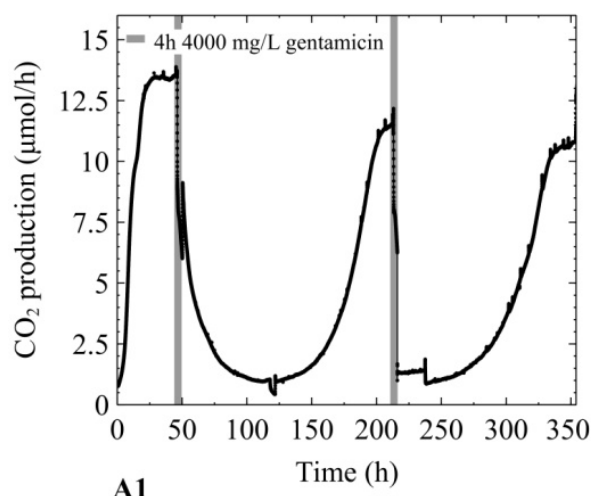

A1

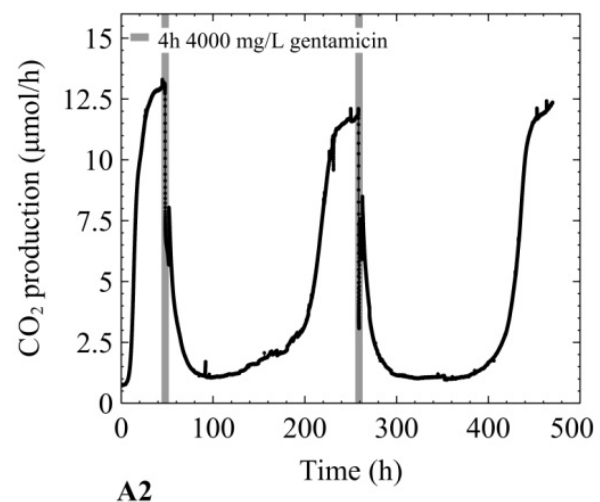

A2
B. pH 7.4 Streptomycin Exposures
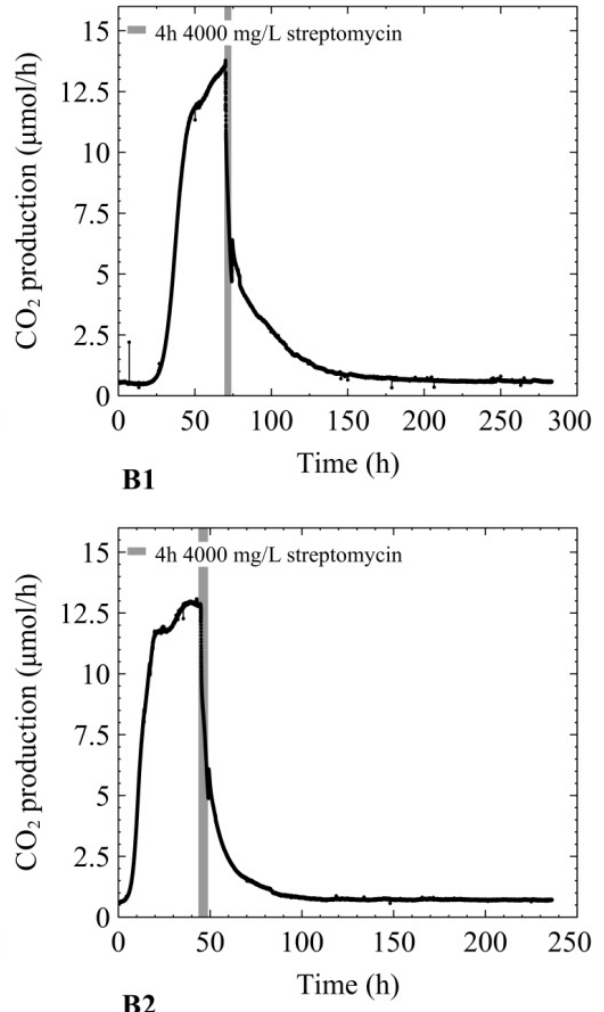

B2

Figure 3.3. Multispecies biofilms were grown in $0.3 \mathrm{~g} / \mathrm{L} \mathrm{TSB}$ medium until a steady state $\mathrm{CO}_{2}$ output was reached prior to being exposed for $4 \mathrm{~h}$ to $4000 \mathrm{mg} / \mathrm{L}$ of either gentamicin ((Column A: (A1 and A2)) or streptomycin (Column B: (B1 and B2))at pH 7.4. 

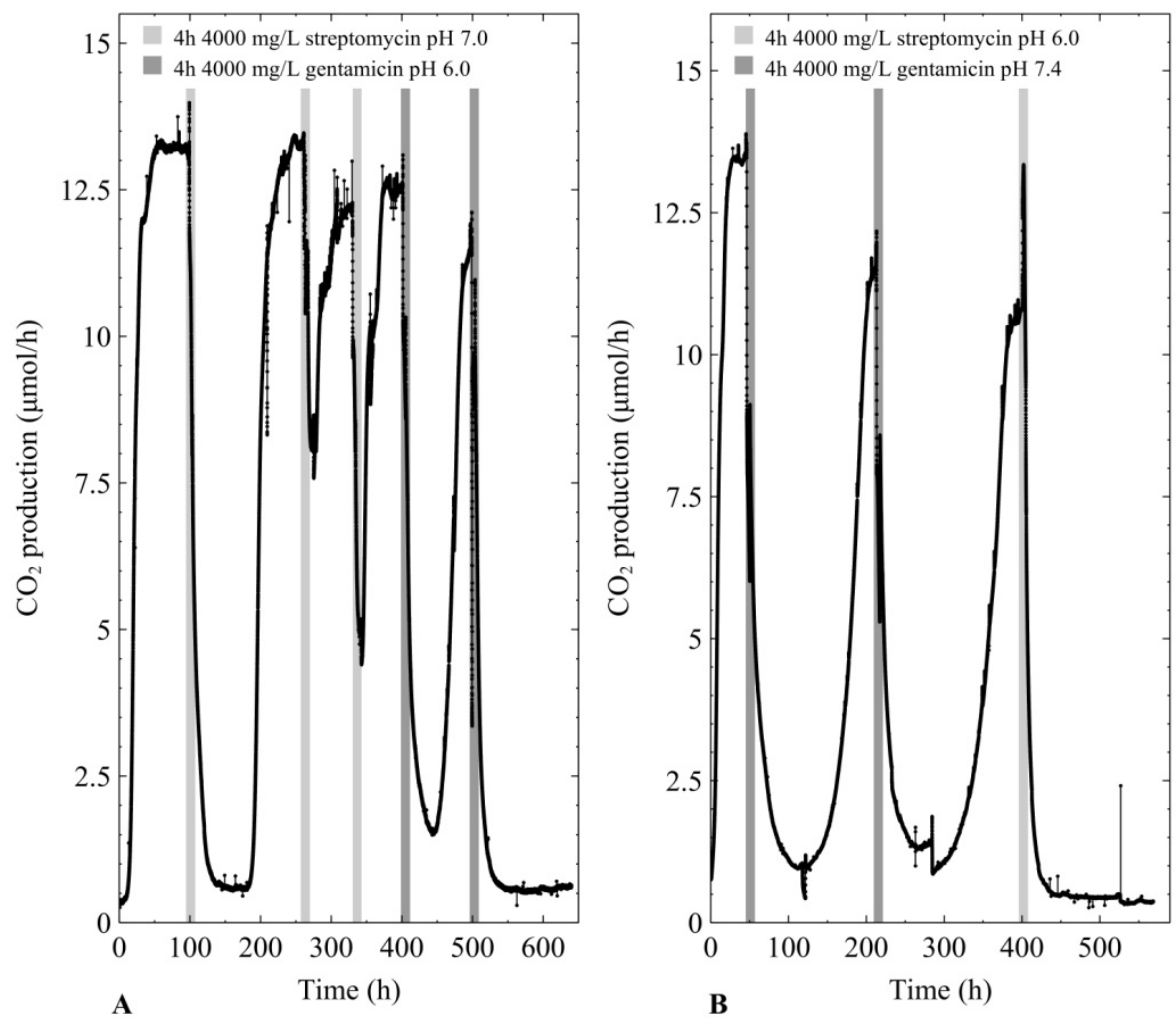

Figure 3.4. Multispecies biofilms grown in $0.3 \mathrm{~g} / \mathrm{L} \mathrm{TSB}$ medium and exposed to (A) $4000 \mathrm{mg} / \mathrm{L}$ streptomycin at pH 7.03 times, followed by two subsequent $4000 \mathrm{mg} / \mathrm{L}$ gentamicin exposures at $\mathrm{pH} 7.0$ (B) $4000 \mathrm{mg} / \mathrm{L}$ gentamicin at pH 7.4, followed by one $4000 \mathrm{mg} / \mathrm{L}$ streptomycin exposure at $\mathrm{pH} 6.0$.

\subsubsection{Cross-resistance experiments}

Antibiotic MIC experiments performed on the effluent of biofilms exposed to streptomycin demonstrated that following repeated exposures, the biofilm effluent streptomycin MIC increased. Thus, it was hypothesized that if survival of the biofilms exposed to streptomycin at pH 7.0 was due to a mechanism of resistance common to aminoglycosides (such as antibiotic efflux pumps or reduced outer membrane permeability (Moore and Flaws, 2011), the biofilms would be able to resist gentamicin as well. A PAO1 biofilm was exposed to three sequential challenges with streptomycin at $\mathrm{pH} 7.0$ and was able to recover each time. Streptomycin was replaced with gentamicin for a fourth exposure, after which the biofilm again recovered to steady state (Fig. 3.4A), although effluent cells collected during this steady state indicated no increase resistance to gentamicin (ie. no change in MIC). When the same biofilm was exposed a second time to gentamicin, no recovery was observed. A similar experiment was performed in which a biofilm was initially exposed to two sequential gentamicin challenges at $\mathrm{pH} 7.4$, prior to a 
streptomycin challenge at $\mathrm{pH}$ 6. The biofilm was able to survive the initial gentamicin exposures but did not show cross resistance to streptomycin (Fig. 3.4B).

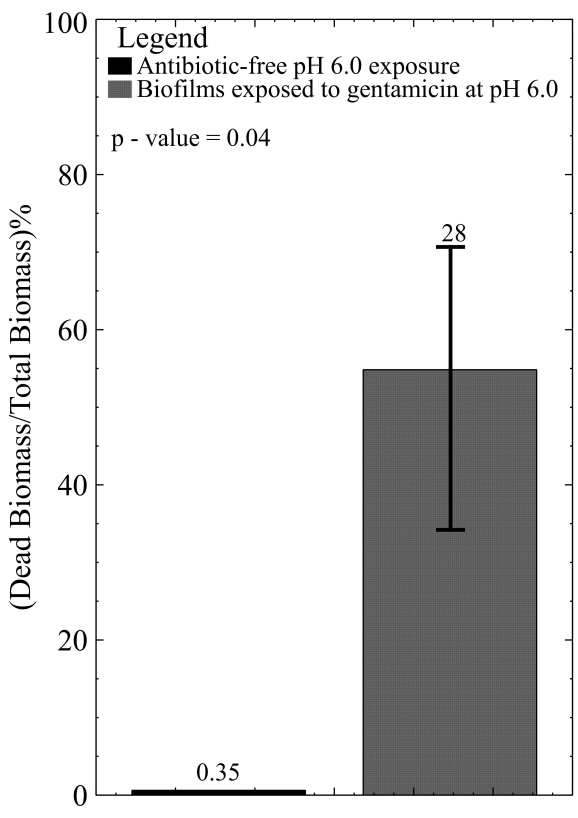

A

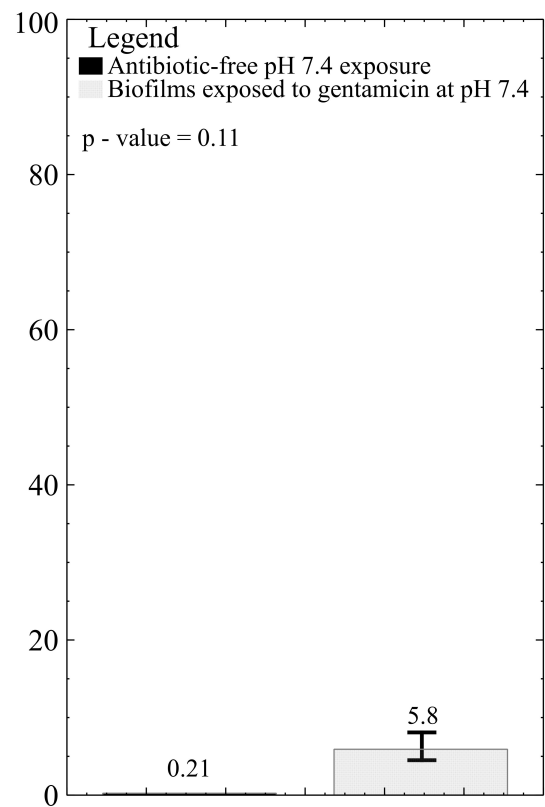

B
Flow Cell Biofilms

Figure 3.5. Multispecies biofilms that were grown in flow cells in $0.3 \mathrm{~g} / \mathrm{L}$ TSB were stained with Syto9 and Propidium lodide for visualization of live versus dead biomass composition. (A) The pH 6.0 control showed an average of less than $0.5 \%$ dead biomass compared to the total biomass and the gentamicin exposures at $\mathrm{pH} 6.0$ had an average of $55 \%$ dead biomass in the biofilm at $168 \mathrm{~h}$ after exposure. (B) The $\mathrm{pH} 7.4$ control showed an average of less than $0.5 \%$ dead biomass compared to the total biomass and the antibiotic treated biofilms had $6 \%$ dead biomass at $168 \mathrm{~h}$ after exposure.

\subsubsection{Live dead staining}

Following antibiotic treatments in CEMS microscopy was performed in order to corroborate the metabolic results that the biofilms were responding differently to the antibiotic treatments at different $\mathrm{pH}$. Using gentamicin as the antibiotic the proportion of live and dead biomass following exposures at $\mathrm{pH} 6.0$ and 7.4 were compared. It was of interest whether there was sufficient evidence to suggest that the mean percentage of dead biomass was greater $168 \mathrm{~h}$ after the biofilm is exposed to gentamicin at $\mathrm{pH} 6.0$ and $\mathrm{pH} 7.4$ compared to their respective antibiotic-free controls. Based on the metabolic data obtained from CEMS it was hypothesized that there would not be a significant difference in dead biomass seen in biofilms exposed to gentamicin at $\mathrm{pH} 7.4$ compared to the control after 7 days, since these biofilms were able to recover following gentamicin exposures (Fig. 3.3 A1-A2). For pH 6.0 gentamicin exposures it was hypothesized that there would be a significant difference in dead biomass compared to the 
$\mathrm{pH} 6.0$ control, as no recovery was seen even with up to 7 days of the biofilms being fed with antibiotic free growth medium (Fig. 3.1A1-A2). The COMSTAT analysis indeed demonstrated that there was a difference in dead biomass at $168 \mathrm{~h}$ after exposure at $\mathrm{pH} 6.0$ but no difference at pH 7.4 compared to their respective controls (Fig. 3.5).

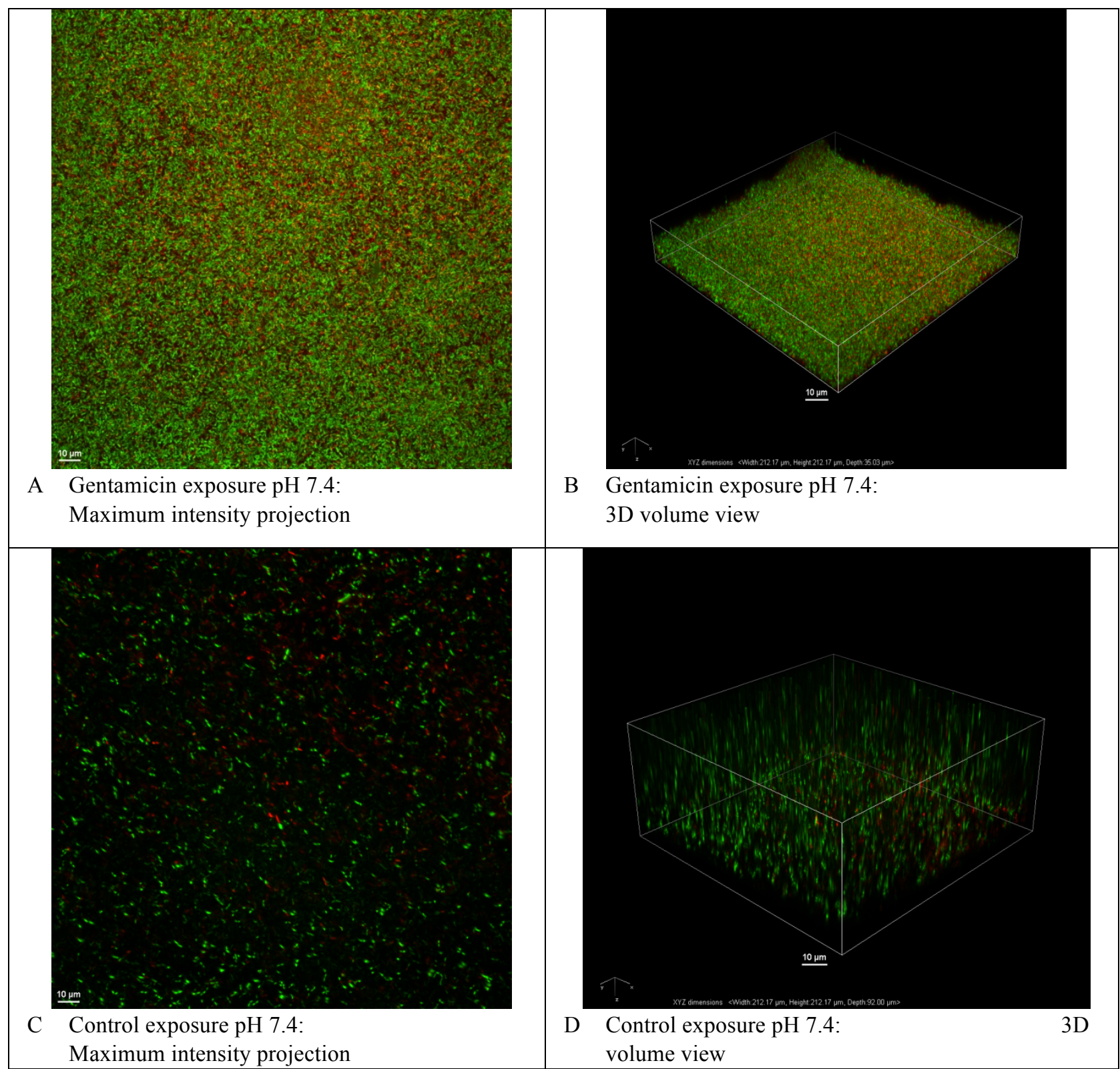

Figure 3.6. Single CLSM micrographs representative of the multispecies biofilms stained with Syto 9 and propidium iodide. Biofilms $168 \mathrm{~h}$ after 4-h $4000 \mathrm{mg} / \mathrm{L}$ gentamicin exposure at $\mathrm{pH} 7.4$ in $0.3 \mathrm{~g} / \mathrm{L} \mathrm{TSB}$ medium (A and B) and $168 \mathrm{~h}$ after a 4-h antibiotic-free $\mathrm{pH} 7.4$ exposure in $0.3 \mathrm{~g} / \mathrm{L} \mathrm{TSB}$ medium (C and D). 
CLSM projections of the multispecies biofilms exposed to $4000 \mathrm{mg} / \mathrm{L}$ of gentamicin demonstrate that the when the biofilms were exposed to the antibiotic at $\mathrm{pH} 6.0$ a higher proportion of the biofilms were stained with propidium iodide $168 \mathrm{~h}$ after exposure compared to exposures at $\mathrm{pH}$ 7.4 and either of the controls (Fig. 3.6 and 3.7).

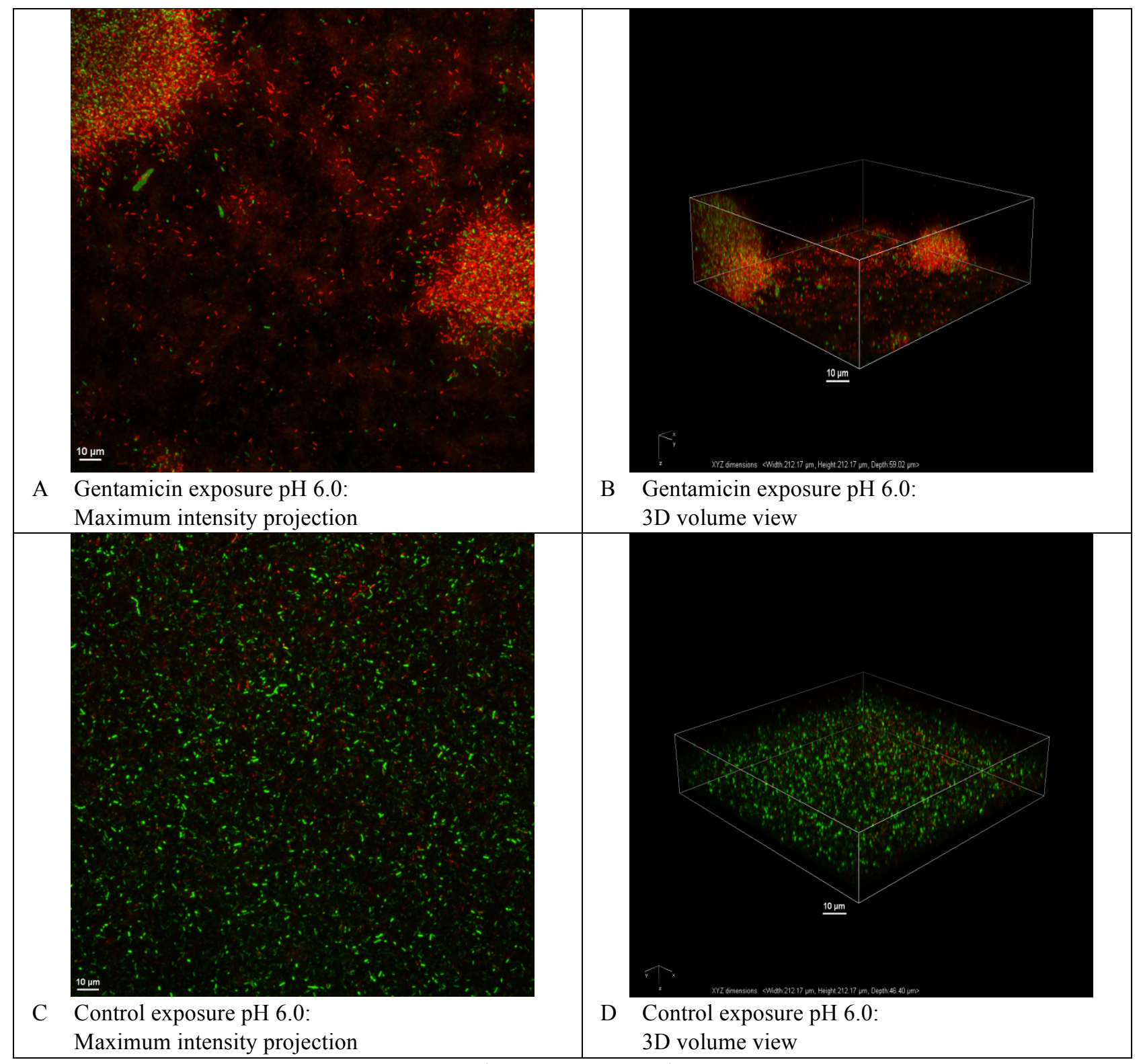

Figure 3.7. Single CLSM micrographs representative of the multispecies biofilms stained with Syto 9 and propidium iodide. Biofilms $168 \mathrm{~h}$ after 4-h $4000 \mathrm{mg} / \mathrm{L}$ gentamicin exposure at pH 6.0 in $0.3 \mathrm{~g} / \mathrm{L} \mathrm{TSB}$ medium ( $\mathrm{A}$ and B) and $168 \mathrm{~h}$ after a 4-h antibiotic-free $\mathrm{pH} 6.0$ exposure in $0.3 \mathrm{~g} / \mathrm{L}$ TSB medium ( $C$ and $\mathrm{D}$ ).

\subsubsection{Metagenome analysis results}

The metagenome data of the planktonic, biofilm, and biofilm effluent showed that there was a multispecies culture of bacteria, with the main bacteria present being $P$. aeruginosa and $S$. 
maltophilia (determined by the species most used to annotate the metagenome data). The percentages at which the two genera (Pseudomonas:Stenotrophomonas) were present in the culture mixtures were as follows: 98:1 (planktonic); 70.3:22.4 (biofilm); 70.2:21.9 (effluent).

\subsection{Discussion}

The efficacy of aminoglycosides is $\mathrm{pH}$ dependent (Xiong et al., 1996). One common property of all aminoglycosides is that they have a net positive charge at physiological $\mathrm{pH}$ of 7.4 due to multiple amino groups with high $\mathrm{pKa}$ (Damper and Epstein, 1981). The physiological $\mathrm{pH}$ of blood is between $\mathrm{pH} 7.34-7.45$ (Islam, 2013), though throughout the body there is variation in the $\mathrm{pH}$ from 5.5-7.4 in various tissues (Culebras, 1996). For example, the lungs of a healthy individual typically have a $\mathrm{pH}$ around 6.15 , while cystic fibrosis patients exhibit an even lower $\mathrm{pH}$ in the lungs. Tate et al. (2002) measured an average $\mathrm{pH}$ of 5.67 in the lungs of CF patients. Thus, for this study we wanted to test how high doses of antibiotics would affect whole-biofilm metabolism when tested at different $\mathrm{pH}$. The internal $\mathrm{pH}$ of $P$. aeruginosa has been found to be near neutral $\mathrm{pH}$ and bacteria have many mechanisms in place to maintain their internal $\mathrm{pH}$ with perturbations in external pH (Whooley and McLoughlin, 1983; Slonczewski et al., 2009). The charge difference across the bacterial membrane is affected by electrical components and chemical components such as changes in proton concentration (Damper and Epstein, 1981). At the three $\mathrm{pH}$ tested in this study ( $\mathrm{pH}$ 6.1, 7.0, and 7.4) aminoglycosides are positively charged and should experience a driving force into the bacterial cells due to the negative interior (Damper and Epstein, 1981). Whooley and McLoughlin 1983 demonstrated that the electrical potential difference across the membrane of $P$. aeruginosa does not change drastically with changes in $\mathrm{pH}$ from $\mathrm{pH}<6.5->8.0(-165 \mathrm{mV}$ to $-190 \mathrm{mV})$. In addition, and Mates (1982) demonstrated that for aminoglycoside uptake an electrical potential of at least $-75-90 \mathrm{mV}$ is required in Staphylococcus aureus; at a membrane potential $-90 \mathrm{mV}$ gentamicin uptake and killing was drastically reduced compared to a membrane potential of $-130 \mathrm{mV}$. Assuming the electrical potential difference reported by Whooley and McLoughlin 1983 is correct it would follow that gentamicin and streptomycin uptake and killing can occur at $\mathrm{pH} 6, \mathrm{pH} 7.0$ and at $\mathrm{pH}$ 7.4.

Although, multiple studies have demonstrated that there is reduced aminoglycoside uptake and killing at acidic pH in planktonic systems (Damper and Epstein, 1981; Xiong et al., 1996). 
Following the logic of previous studies, streptomycin and gentamicin antibiotic exposures at acidic $\mathrm{pH}$ were expected to have a less marked effect compared to the same exposures at higher $\mathrm{pH}$. Contradictory to what has been seen in other studies, at $\mathrm{pH} 6.0$ there was increased susceptibility observed in CEMS after both streptomycin and gentamicin exposures. Previous work has reported reductions in aminoglycoside efficacy at acidic pH (Bryan, L.E., 1983; Xiong et al., 1996; Gudmundsson et al., 1991). One might expect that if biofilms are susceptible to high doses of the aminoglycosides tested at acidic $\mathrm{pH}$ then they would also be susceptible to the antibiotics at neutral $\mathrm{pH}$. The optimal $\mathrm{pH}$ range for $P$. aeruginosa has been reported to be between $\mathrm{pH}$ 6.6-7.0 (Soccor, C. R., 2013). When the external pH and the internal $\mathrm{pH}$ are equal, or there is little difference in $\mathrm{pH}$ across the membrane, the main component of the proton motive force is the electrical potential (Khan and Macnab, 1980). It could then be presumed the aminoglycosides would be experiencing a driving force into the cell while there is a larger charge difference across the bacterial membrane. However, at $\mathrm{pH} 7.0$ the biofilms were able to recover from both streptomycin and gentamicin exposures. Interestingly, our antibiotic-free experiments demonstrated that even when fed growth medium between $\mathrm{pH} 5.0$ to 8.0 , the biofilms will adjust its $\mathrm{pH}$ towards its optimal range. It is plausible that the biofilms in this study exposed to aminoglycosides at $\mathrm{pH} 6.0$ and 7.4 were experiencing a dual stress from the antibiotic and the change in $\mathrm{pH}$ of the media preventing them from overcoming the antibiotic exposures. If the biofilm was working to keep their local $\mathrm{pH}$ close to neutral this may have affected their ability to resist the aminoglycoside exposures.

One study demonstrated that the internal $\mathrm{pH}$ cannot be maintained when bacteria are growing under stress conditions (Booth, 1985). In addition, changes in external $\mathrm{pH}$ have been found to trigger transient changes of internal $\mathrm{pH}$, which may produce changes in both the expression of certain genes such as the Escherichia coli SOS response and the activity of certain enzymes (Dri \& Moreau, 1994). Thus, it is plausible that when the biofilms were exposed to streptomycin at $\mathrm{pH} 6.0$ and $\mathrm{pH} 7.4$, and gentamicin at $\mathrm{pH} 6$, they were too stressed to properly adjust the $\mathrm{pH}$ closer to their optimal range, preventing survival following antibiotic exposure.

The experiments from this study revealed differences in the efficacy of high doses of streptomycin and gentamicin that did not follow trends previously described in literature. One study demonstrated that in $P$. aeruginosa bacterial killing by the aminoglycoside amikacin at $\mathrm{pH}$ 
5.5 was far lower than at $\mathrm{pH} 7.4$, but was less so for netilmicin, and at $\mathrm{pH} 5.5$ amikacin and netilmicin had practically no bactericidal effect (Xiong et al., 1996). For high doses of gentamicin specifically, our data demonstrated reduced antibiotic efficacy at $\mathrm{pH} 7.0$ and 7.4 compared to $\mathrm{pH} 6.0$.

Effluent cells did not show an increase in gentamicin MIC at pH's 7.0 and 7.4 following recovery of the first antibiotic exposures (Table 3.1). Since changes in biofilm behaviour or resistance was seen following survival and recovery to gentamicin exposures adaptive resistance may be one potential mechanism that the biofilms survived the gentamicin exposures. Adaptive resistance is when there is a transitory reduction in antibiotic susceptibility that may be related to down-regulation of aminoglycoside uptake during the period of energy-dependent transport and to a reduced proton motive force (Mingeot-Leclercq et al.; 1999; Xiong, 1996). Presumably, if adaptive resistance is happening in the presence of gentamicin at $\mathrm{pH} 7.4$ it would be able to occur with streptomycin also: streptomycin has a net charge of +3 (Lerman, 1964; Eisenberg et al., 1984) and gentamicin has a charge of +3.46 at physiological $\mathrm{pH}$ (Kadurugamuwa et al., 1993). Although, aminoglycosides have been shown to elicit various responses under similar conditions (Davies and Davis, 1968; Milman and Dahlager, 1978). For E. coli the cytoplasmic interior is maintained near $\mathrm{pH} 7.5$, thus in media at $\mathrm{pH} 7.4$ the $\mathrm{pH}$ difference across the membrane contributes much less to the proton motive force (PMF), and most of the PMF is expressed by the electrical potential (Damper, 1981). For P. aeruginosa the intracellular pH increases with increasing extracellular $\mathrm{pH}$, reaching a maximum of $\mathrm{pH} 7.0$ when the extracellular $\mathrm{pH}$ was 7.5 or above, though, the study did not find a relationship between electrical potential and external $\mathrm{pH}$ (Whooley and McLoughlin, 1983). One study demonstrated that aminoglycoside uptake can occur even in the absence of electrical potential, and is likely up-taken as a result of ATP (Fraimow et al., 1991). Thus, even if the electrical potential is affected, it is still possible for aminoglycoside uptake and killing.

In an attempt to gain insight into the potential antibiotic resistance mechanisms involved in streptomycin or gentamicin resistance, cross resistance was tested between antibiotics. If resistance was due to non-specific multi-drug/-aminoglycoside resistance mechanisms, then once resistance developed at a particular $\mathrm{pH}$ for one aminoglycoside, resistance to the other would be expected. Sustained cross resistance was not seen when switching from streptomycin at $\mathrm{pH} 6.0$ to 
gentamicin at $\mathrm{pH} 7.0$ (Fig. 3.4A). Thus, it is unlikely that the resistance mechanisms are a result of increased efflux activity (Moore and Flaws, 2011) or hyper-expressed efflux pumps. Since cross resistance was not seen between the cultures, it is possible that aminoglycoside modifying enzymes played a role in the resistance of the biofilms to the antibiotics since there are no aminoglycoside modifying enzyme can modify both streptomycin and gentamicin (Shaw et al., 1993). Metagenomic analysis did reveal the presence of the aminoglycoside modifying enzyme genes aph(6)-Id and aph(3)-IIb which can potential confer resistance to streptomycin and gentamicin (Hachler et al., 1996; Shaw et al., 1993; Chiou and Jones, 1995). Presence of a streptomycin modifying enzyme may have aided in streptomycin resistance at $\mathrm{pH} 7$ when biofilm adaptations can be seen. Though, it is unclear why the resistance gene would not aid the biofilms at $\mathrm{pH} 6.0$ and $\mathrm{pH}$ 7.4. As for the gentamicin exposed biofilms, there was no apparent increase in resistance to gentamicin following subsequent exposures. If the level of modifying enzymes were to increase throughout the population following subsequent exposures, changes in biofilm behaviour and the effluent MIC would be expected.

Potential explanations for survival following gentamicin treatments with no increases in MIC may be due to the presence of persister cells in the biofilm or lack of antibiotic penetration through the biofilm leaving some cells unexposed (Stewart, 2002). It was found that gentamicin and erythomycin do not penetrate well though alginate (Kumon et al. 1994). However, another study found that $P$. aeruginosa (strain PAO1) does not produce alginate in biofilm (Wozniak et al., 2003). Thus we cannot simply draw on those conclusions that lack of penetration though the biofilm is occurring in our streptomycin and gentamicin exposed biofilms.

Microscopic analysis of the biofilms exposed to gentamicin at $\mathrm{pH} 6.0$ and $\mathrm{pH} 7.4$ (Fig. 3.5) showed similar trends to what was observed from the metabolic experiments in CEMS. At $\mathrm{pH}$ 6.0 gentamicin exposures showed an increase in dead biofilm biomass compared to the $168 \mathrm{~h}$ controls whereas the $\mathrm{pH} 7.4$ gentamicin exposures did not. In addition, CLSM micrographs visually depict a higher proportion of red-stained cells in biofilms exposed to gentamicin at $\mathrm{pH}$ 6.0 (Fig. 3.7). The red-staining occurs from propidium iodide uptake, which indicates either dead or compromised cells (Stiefel et al., 2015). This does not confirm that all the cells within the biofilm are dead following the $\mathrm{pH} 6.0$ gentamicin treatments, though this data along with the 
CEMS results indicates that there is increased susceptibility of the biofilms to gentamicin at $\mathrm{pH}$ 6.0 .

\subsection{Conclusion}

The aminoglycosides used in this study vary in their effectiveness at the $\mathrm{pH}$ tested. Streptomycin antibiotics allow for adaptation or resistance from the biofilm with subsequent exposures at $\mathrm{pH}$ 7.0. However, following gentamicin exposures even when the biofilms can survive the exposure there is no indication that the biofilms are developing resistance to the antibiotic. The results of our study differ from previous work in that there is no linear increase with $\mathrm{pH}$ and aminoglycoside killing in biofilm that has been demonstrated in planktonic systems. Future studies will be performed to separate the effects of $\mathrm{pH}$ versus the antibiotic to determine if there was indeed a dual stress occurring on the biofilm during these exposures that affected aminoglycoside susceptibility. In addition, experiments with a $P$. aeruginosa monoculture deficient in antibiotic efflux will be performed to help infer whether antibiotic efflux or antibiotic uptake is more affected at the different $\mathrm{pH}$ tested.

The next chapter will look at the intrinsic potential of the cultures used throughout this study in order to determine what potential mechanisms of resistance may have led to biofilm survival to high dose aminoglycoside exposures. 
Chapter 4

Multispecies biofilms: potential benefits for antibiotic resistance 


\section{Multispecies biofilms: potential benefits for antibiotic resistance}

\subsection{Introduction}

In natural and clinical settings biofilms are formed from multispecies communities living at high population densities. Living in a biofilm offers many benefits to bacteria including increased rates of genetic exchange compared to planktonic cultures (Madsen et al., 2012). High rates of genetic exchange and transfer can occur due to the close proximity of bacteria within a biofilm (Molin and Tolker-Nielsen, 2003), often leading to a degree of cooperation (Davey and O'Toole, 2000). Harbouring of genes from a subset of members in a multispecies community can be beneficial to a biofilm in the right circumstances. In clinical settings, it can be detrimental for a patient given antibiotics to treat their chronic infection if members within the biofilm are carriers of antibiotic resistance genes. Antibiotic resistance has become more prevalent with the increased use in antibiotics over the past 50 years as they are the main method of treating bacterial infections (Beceiro et al., 2013). There are a number of antimicrobial resistance mechanisms that bacteria can employ to resist each class of antibiotics. Antibiotic resistance mechanisms can be inherent or acquired through acquisition of resistance genes from other bacteria (Tenover, 2006). Understanding the genetic potential of a bacterial culture or community can help determine reasons for high levels of antibiotic resistance and aid in guiding future investigations.

Billions of dollars in health care costs are incurred each year from high rates of nosocomial infections that are difficult to treat due to the development of chronic wounds and increasing numbers of antibiotic resistant pathogens (Bryers et al., 2008; Beceiro et al., 2013). Opportunistic pathogens, such as Pseudomonas aeruginosa and Stenotrophomonas maltophilia, are implicated in many different diseases (Stover et al., 2000; Araoka et al., 2010). Pseudomonas and Stenotrophomonas spp have been co-isolated from a number of chronic wound types, such as the cystic fibrosis lung, venous leg ulcers and hospital acquired pneumonia (Goss et al., 2002; Dowd et al., 2008; Tseng et al., 2009). The majority of the experiments in this dissertation were performed on multispecies biofilms containing Pseudomonas and Stenotrophomonas exposed to high doses of aminoglycoside antibiotics. Hence, there was interest in uncovering their intrinsic potential to persist when exposed to these antibiotics. 
In the previous chapters, multispecies biofilms and pure culture environmental isolates demonstrated high levels of tolerance and resistance to either gentamicin and/or streptomycin antibiotics. Particular genes of interest that relate to aminoglycoside resistance include: $16 \mathrm{~S}$ ribosomal mutations ( $r p s L)$, efflux pump mutations (MexXY, MexAB), the presence of aminoglycoside modifying enzymes, and porin/membrane modifications (Paulander et al., 2009; Moore and Flaws, 2011). Given the observed tolerance to streptomycin and gentamicin it was hypothesized that multispecies cultures of P. aeruginosa and S. maltophilia would contain intrinsic resistance to these aminoglycosides. The purpose of this study was to determine how genetics and community member abundance changed when in different physiological states, different growth media, and following antibiotic treatments. The following methods of genetic analysis were used to test for the presence of antibiotic resistance genes in the cultures used in this study: (1) Polymerase chain reaction (PCR) for monitoring of specific genes in both multispecies and pure cultures, (2) genome analysis for pure cultures of the individual species in the multispecies cultures, and (3) metagenomic analysis of the multispecies culture to observe intrinsic potential and community composition dynamics under the conditions tested.

\subsection{Materials and Methods}

\subsubsection{Strains and culture conditions}

The bacterial strains used in PCR sequencing experiments were three Gram-negative environmental isolates (Enterobacter asburiae, Enterobacter sp., and Pseudomonas putida) that were previously cultured and isolated from a washroom sink drain (Ghadakapour et al., 2014), and a multispecies culture containing Pseudomonas aeruginosa and Stenotrophomonas maltophilia strains. A strain of E. coli (EMG A20) was used as a negative control for PCR amplification with the streptomycin modifying enzyme primers only. The bacterial cultures used for genomic sequencing included bench cultures and freezer stocks of the multispecies culture. All environmental isolates and the multispecies cultures were kept at room temperature on $3 \mathrm{~g} / \mathrm{L}$ TSA plates. Analyses were also done with the multispecies cultures from freezers stocks kept at $20^{\circ} \mathrm{C}$ prior to being grown overnight in either $3 \mathrm{~g} / \mathrm{L}$ TSB (EMD Chemicals, Billerica, MA, USA.) or a defined growth medium with final concentrations of $1.51 \mathrm{mM}\left(\mathrm{NH}_{4}\right)_{2} \mathrm{SO}_{4}, 3.37 \mathrm{mM}$ $\mathrm{Na}_{2} \mathrm{HPO}_{4}, 2.20 \mathrm{mM} \mathrm{KH}_{2} \mathrm{PO}_{4}, 179 \mathrm{mM} \mathrm{NaCl}, 0.1 \mathrm{mM} \mathrm{MgCl}{ }_{2} \cdot \mathrm{H}_{2} \mathrm{O}, 0.01 \mathrm{mM} \mathrm{CaCl}_{2} \bullet 2 \mathrm{H} 2 \mathrm{O}, 0.001$ $\mathrm{mM} \mathrm{FeCl}_{3}$ (Clark and Maaløe, 1967), with $1 \mathrm{mM}$ citrate as the carbon source. 


\subsubsection{Biofilm cultivation}

All biofilms were inoculated with $1 \mathrm{ml}$ of an overnight culture from the $-20^{\circ} \mathrm{C}$ freezer stocks kept in either $3 \mathrm{~g} / \mathrm{L}$ TSB or $1 \mathrm{mM}$ citrate defined medium. Biofilms were cultivated aerobically at $25^{\circ} \mathrm{C}$ in the CEMS apparatus (Kroukamp et al., 2010) with a continuous flow of growth medium at a rate of $15 \mathrm{ml} / \mathrm{h}$. Biofilms were grown in either $0.3 \mathrm{~g} / \mathrm{L}$ TSB medium or a $1 \mathrm{mM}$ citrate defined medium.

\subsubsection{Biofilm Antibiotic Exposures}

The multispecies biofilms inoculated from the $-20{ }^{\circ} \mathrm{C}$ freezer stock were grown to steady state levels for 5 days prior to a $4 \mathrm{~h} 4000 \mathrm{mg} / \mathrm{L}$ streptomycin exposure in $0.3 \mathrm{~g} / \mathrm{L} \mathrm{TSB}$ at $\mathrm{pH} 6.0$. Previous $4000 \mathrm{mg} / \mathrm{L}$ streptomycin exposures on early steady state multispecies biofilms from the $-20{ }^{\circ} \mathrm{C}$ freezer stock at $\mathrm{pH} 6.0 \mathrm{did}$ not result in recovery following antibiotic exposure. Thus, the biofilm was grown for 5 days prior to the antibiotic exposure as it was shown previously that older biofilms can recover from the streptomycin exposures. From one of the replicates, the effluent was collected and its DNA extracted for subsequent genomic analysis once the biofilm exposed to the antibiotic recovered back to steady state levels.

\subsubsection{Whole-biofilm and biofilm effluent sample preparation for DNA extraction}

The multispecies biofilm that was grown to steady state levels in CEMS was sacrificed after $72 \mathrm{~h}$ for DNA extraction following a similar protocol used for whole biofilm protein extraction (Kroukamp et al., 2010). In essence, the entire bulk fluid ( $\sim 2 \mathrm{ml})$ was drained from the CEMS into a waste container. Following removal of the bulk liquid, the remaining attached biofilm was removed by injecting $2 \mathrm{ml}$ of a $0.1 \mathrm{M} \mathrm{NaOH}$ solution preheated to $60^{\circ} \mathrm{C}$ and collected into a 15 $\mathrm{ml}$ polyethylene tube. To ensure full biofilm collection and removal, another $2 \mathrm{ml}$ of $0.1 \mathrm{M}$ $\mathrm{NaOH}$ was injected into the biofilm growth chamber of the CEMS and kept in a $60^{\circ} \mathrm{C}$ incubator

for $1 \mathrm{~h}$ prior to being flushed into the same $15 \mathrm{ml}$ polyethylene tube containing the first wash of biofilm.

Biofilm effluent samples were collected prior to biofilm sampling for $18 \mathrm{~h}$ into sealed sterile flasks on ice. The biofilm fraction and each biofilm effluent sample were then separated into 1 $\mathrm{ml}$ Eppendorf tubes and centrifuged for $5 \mathrm{~min}$ at $12,000 \mathrm{xg}$. The supernatant was discarded and all pellets were collected into two $1.5 \mathrm{ml}$ Eppendorf tubes prior to DNA extraction. 


\subsubsection{Preparation of genomic DNA for PCR and genome sequencing}

Planktonic cultures were grown in $3 \mathrm{~g} / \mathrm{L}$ TSB medium and biofilms were grown in $0.3 \mathrm{~g} / \mathrm{L}$ TSB medium. The genomic DNA of the planktonic cultures, whole-biofilm, and biofilm effluent collected from pre- and post-streptomycin exposed steady state biofilms were extracted using the MoBio UltraClean ${ }^{\circledR}$ Microbial DNA Extraction Kit (MoBio Laboratories INC., Carlsbad, CA, USA) following the manufacturer's protocol. Once extracted, the microbial DNA was kept at -20 ${ }^{\circ} \mathrm{C}$ until further testing was performed.

\subsubsection{Preparation of plasmid DNA for PCR amplification}

A plasmid DNA extraction kit was used to determine if the cultures contained aminoglycoside modifying enzymes on plasmids. Planktonic cultures were grown in $3 \mathrm{~g} / \mathrm{L}$ TSB medium prior to use with a Zyppy ${ }^{\mathrm{TM}}$ Plasmid Miniprep Kit (Zymo Research Corporation, Irvine, CA, USA) following the manufacturer's protocol. Once extracted, the plasmid DNA was kept at $-20{ }^{\circ} \mathrm{C}$ prior to PCR amplification with the aminoglycoside modifying enzyme primers listed in Table 4.1.

\subsubsection{Primers and PCR amplification}

Each $50 \mu 1$ PCR reaction contained $1 \mu 1$ of genomic DNA, 25 pmol of both forward and reverse primers (Table 4.1), $6.875 \mu \mathrm{g}$ of BSA, $200 \mu \mathrm{M}$ of each of the four dNTPs, and 2.5 units of Taq polymerase in Taq buffer (10 mM Tris- $\mathrm{HCl} \mathrm{pH} \mathrm{9.0,50} \mathrm{mM} \mathrm{KCl,} 1.5 \mathrm{mM} \mathrm{MgCl} 2)(\mathrm{New}$ England BioLabs, Pickering, ON, Canada). PCR product size was established on a $1 \%$ agarose gel containing SYBR Safe DNA gel stain (Invitrogen, Burlington, ON, Canada). PCR products were quantified against a standard curve prepared with $1 \mu 1,2 \mu 1$, and $4 \mu \mathrm{l}$ of a 100-bp molecular weight ladder (MBI Fermentas, Amherst, NY, USA).

In order identify the bacterial isolates used in this study, the conserved region of 16S rRNA was amplified by PCR using the bacteria-specific forward and reverse primers (U341F and U758R, respectively (Table 4.1) that produce a 418-bp fragment corresponding to nucleotides 341 to 534 of the 16S sequence in Escherichia coli (Rolleke et al., 1996). The samples were first denatured at $96{ }^{\circ} \mathrm{C}$ for $5 \mathrm{~min}$ and thermocycling at $94{ }^{\circ} \mathrm{C}$ for $1 \mathrm{~min}$. An initial annealing temperature of 65 ${ }^{\circ} \mathrm{C}$ was lowered by $1{ }^{\circ} \mathrm{C}$ every cycle for a total of 20 cycles using an elongation time of $3 \mathrm{~min}$ at $72{ }^{\circ} \mathrm{C}$. Additional $15-20$ cycles were performed at a $55^{\circ} \mathrm{C}$ annealing temperature (Yeung et al., 
2011). PCR product size was established on a $1 \%$ agarose gel containing SYBR Safe DNA gel stain (Invitrogen, Burlington, ON, Canada). PCR products were quantified against a standard curve prepared with $1 \mu 1,2 \mu 1$, and $4 \mu 1$ of a 100-bp molecular weight ladder (MBI Fermentas, Amherst, NY, USA).

PCR products were sent for sequencing at the Centre for Applied Genomics at SickKids in Toronto using the Applied Biosystems SOLiD 3.0 System. The resulting forward and reverse nucleotide sequences were used to create a single consensus sequence using the BioEdit Sequence Alignment Editor (Version 7.0.9.0; Hall, 1999). The consensus sequence was subjected to a BLAST search using the NCBI database (http://blast.ncbi.nlm.nih.gov/Blast.cgi).

Primers were obtained for the following gene sequences to determine whether the isolates contained genes for streptomycin modifying enzymes: ant (3")b, aph (3")c, aph (6)-1dd, aph (6)1ce, ant (6)f (Overbeek et al., 2002), and strAB (containing the SME genes aph (3")-Ib and aph (6)-Id) (Han et al., 2003). The PCR reaction settings were performed as previously described (Overbeek et al., 2002; Han et al., 2003).

\begin{tabular}{|c|c|c|c|c|}
\hline Primer & Forward Sequence 5" $\rightarrow 3 "$ & Reverse Sequence 5" $\rightarrow 3 "$ & $\begin{array}{l}\text { Fragment } \\
\text { Size (bp) }\end{array}$ & $\begin{array}{l}\text { Source of } \\
\text { Reference }\end{array}$ \\
\hline $\begin{array}{l}\mathrm{U} 341 \mathrm{~F} / \\
\mathrm{U} 758 \mathrm{R}\end{array}$ & CCTACGGGAGGCAGC AG & CTACCAGGGTATCTAATCC & 418 & $\begin{array}{l}\text { Rolleke et } \\
\text { al., } 1996\end{array}$ \\
\hline strA & TGAATCGCATTCTGACTGGTT & AAGTTGCTGCCCCATTGA & 301 & $\begin{array}{l}\text { Han et al., } \\
2003\end{array}$ \\
\hline str $\mathrm{B}$ & GGAACTGCGTGGGCTACA & GCTAGATCGCGTTGCTCCTCT & 303 & $\begin{array}{l}\text { Han et al., } \\
2003\end{array}$ \\
\hline strAB & ATCGGTTGATCAATGTCCGT & AAACAAAGCTGCAAAGCGAT & 860 & $\begin{array}{l}\text { Han et al., } \\
2003\end{array}$ \\
\hline ant $\left(3^{\prime \prime}\right)^{b}$ & CAGCGCAATGACATTCTTGC & GTCGGCAGCGACA(C/T)CCTTCG & 295 & $\begin{array}{l}\text { Overbeek } \\
\text { et al., } 2002\end{array}$ \\
\hline $\operatorname{aph}\left(3^{\prime \prime}\right)^{c}$ & GCTCAAAGGTCGAGGTGTGG & CCAGTTCTCTTCGGCGTTAG & 515 & $\begin{array}{l}\text { Overbeek } \\
\text { et al., } 2002\end{array}$ \\
\hline $\operatorname{aph}(6)-1 d^{d}$ & GACTCCTGCAATCGTCAAGG & GCAATGCGTCTAGGATCGAG & 560 & $\begin{array}{l}\text { Overbeek } \\
\text { et al., } 2002\end{array}$ \\
\hline $\operatorname{aph}(6)-1 c^{e}$ & GAGCGCACCTTCGACTATGC & GCCATGGCGTTTACGGCCAG & 248 & $\begin{array}{l}\text { Overbeek } \\
\text { et al., } 2002\end{array}$ \\
\hline ant $(6)^{f}$ & CCATTCATCTCAAAAGTAGC & CTCAACGTATGTAGCAAAGG & 218 & $\begin{array}{l}\text { Overbeek } \\
\text { et al., } 2002\end{array}$ \\
\hline
\end{tabular}

Table 4.1. Primers used for PCR amplification of the $16 \mathrm{~S}$ region of DNA and for streptomycin modifying enzyme detection. 


\subsubsection{Genomic and metagenomic analyses}

The genomic DNA was submitted to The University of Toronto Center for the Analysis of Genome Evolution and Function (CAGEF) for genome sequencing, where the MiSeq Personal Sequencer v2 for the whole genome sequencing was used. Full genome data sets were submitted to the CLoVR main frame software for automated sequence assembly analysis using Velvet (Zerbino, 2010). Once all the genomes were assembled they were submitted to RAST for annotation and subsystem characterizations (Aziz et al., 2008). For the genome and metagenome data, the unassembled data provided by CAGEF were also uploaded to a mg-RAST server (v3.2), which provides annotation, analyses, and metadata on the uploaded metagenomes (Meyer et al., 2008).

\subsection{Results}

\subsubsection{PCR}

The sequencing results confirmed the species identities of cultures used in this study as: $P$. aeruginosa, Sphingobacterium, P. putida, E. absuriae, and an Enterobacter sp. Furthermore, the PCR amplification of the isolate DNA with each of the streptomycin modifying enzyme primers resulted in the identification of one streptomycin modifying enzyme, the aph (3") gene, that was amplified from $P$. putida DNA (Table 4.2). The gene sequence was confirmed with sequencing and BLAST analysis. Additional isolates that produced PCR products were observed as bands of DNA on a gel. However, when these bands of DNA were excised and sent for sequencing the sequences were not identified as streptomycin modifying enzymes. Given that streptomycinmodifying enzymes can be found on plasmids, each strain had their DNA extracted using a plasmid DNA extraction kit. However, none of the cultures produced visible bands of DNA following plasmid DNA extraction.

PCR amplification did not reveal the presence of any streptomycin modifying enzymes in the multispecies cultures tested. In an effort to obtain a better understanding regarding the genetics of the multispecies cultures, more thorough methods of analysis, including whole genome analysis, were performed in order to find novel genes. 


\begin{tabular}{|c|c|c|c|c|c|c|c|}
\hline & \multicolumn{7}{|c|}{ Bacterial Isolates } \\
\hline Primer & $\begin{array}{l}\text { NegativeControl: } \\
\text { E. coli }\end{array}$ & $\begin{array}{l}* P . \\
\text { aeruginosa } \\
\text { Freezer } \\
\text { Stock }\end{array}$ & $\begin{array}{l}* P . \\
\text { aeruginosa } \\
\text { Bench } \\
\text { Culture }\end{array}$ & Sphingobacterium & $\begin{array}{l}P . \\
\text { putida }\end{array}$ & $\begin{array}{l}\text { Enterobacter } \\
\text { sp. }\end{array}$ & $\begin{array}{l}\text { E. } \\
\text { absuriae }\end{array}$ \\
\hline $\begin{array}{l}\operatorname{aph}(6)- \\
1 c\end{array}$ & - & - & - & - & - & - & - \\
\hline ant(6) & - & - & - & - & - & - & - \\
\hline $\operatorname{ant}(3 ”)$ & - & - & - & - & - & - & - \\
\hline $\operatorname{aph}(3 ”)$ & - & - & - & - & + & - & - \\
\hline $\begin{array}{l}\operatorname{aph}(6)- \\
1 d\end{array}$ & - & - & - & - & - & - & - \\
\hline $\operatorname{str} A$ & - & - & - & - & - & - & - \\
\hline strB & - & - & - & - & - & - & - \\
\hline $\operatorname{str} A B$ & - & - & - & - & - & - & - \\
\hline
\end{tabular}

Table 4.2 Gel electrophoresis results combined with product sequencing results for PCR amplicons with the streptomycin modifying enzymes (SME) on the genomic DNA of the multispecies freezer stocks, multispecies bench cultures, the Gramnegative environmental isolates, and a negative control from a known species (E. coli EMG A20). The only PCR product that returned a sequence relating to a SME was the $a p h\left(3^{\prime \prime}\right)$ PCR product amplified from the $P$. putida isolate. *The section of DNA PCR amplified with the U341 and U378 primers identified the freezer cultures and bench cultures as $P$. aeruginosa.

\subsubsection{Whole genome analysis}

The majority of the experimental work was performed on the freezer culture and bench culture that were previously identified from 16S DNA sequencing as P. aeruginosa. Planktonic DNA of both the bench and freezer cultures, as well as a DNA extracted from a biofilm and various biofilm effluent cultures inoculated from the freezer culture were submitted for full genome sequencing. The bench culture was uploaded to the RAST server for genome analysis. From the RAST annotations, the genome sequences obtained revealed that the planktonic culture from the bench stock and PAO1 from Genbank had a similar number of genes present in each subsystems category (Table 4.3). The bench culture was relatively pure with $98 \%$ of Pseudomonas, $0.1 \%$ Stenotrophomonas, $0.4 \%$ Microvirus, and 1.5\% other (Fig. 4.2A). Thus, P. aeruginosa (PAO1) from Genbank was used as the type strain or reference point for this culture. By comparing the genetics of the multispecies bench culture to PAO1 from Genbank the intrinsic potential each could be discerned and differences in subsystem features analyzed. Table 4.3 provides subsystem feature counts of the PAO1 genome uploaded to RAST from Genbank in comparison to the bench culture. There are 18 out of 27 subsystem groups that show variations in the number of genes between the two genomes and 9 subsystem groups where the gene counts are the same. 


\begin{tabular}{|c|c|c|}
\hline Subsystem Features: & PAO1 Genbank & Bench Culture \\
\hline Cofactors, Vitamins, Prosthetic Groups, Pigments & 325 & 329 \\
\hline Cell Wall and Capsule & 199 & 199 \\
\hline Virulence, Disease and Defence & 143 & 138 \\
\hline Potassium Metabolism & 23 & 23 \\
\hline Photosynthesis & 0 & 0 \\
\hline Miscellaneous & 70 & 72 \\
\hline Phages, Prophages, transport elements, Plasmids & 18 & 21 \\
\hline Membrane Transport & 258 & 253 \\
\hline Iron Acquisition and Metabolism & 141 & 128 \\
\hline RNA Metabolism & 201 & 202 \\
\hline Nucleosides and nucleotides & 137 & 137 \\
\hline Protein metabolism & 304 & 271 \\
\hline Cell division and cell cycle & 36 & 36 \\
\hline Motility and chemotaxis & 116 & 114 \\
\hline Regulation and cell signalling & 112 & 111 \\
\hline Secondary Metabolism & 9 & 8 \\
\hline DNA metabolism & 109 & 109 \\
\hline Fatty cids, lipids, and isoprenoids & 213 & 214 \\
\hline Nitrogen metabolism & 88 & 88 \\
\hline Dormancy and sporulation & 2 & 2 \\
\hline Respiration & 160 & 152 \\
\hline Stress response & 190 & 192 \\
\hline Metabolism and aromatic compounds & 119 & 119 \\
\hline Amino acids and derivatives & 688 & 685 \\
\hline Sulfur metabolism & 121 & 119 \\
\hline Phosphorous metabolism & 63 & 64 \\
\hline Carbohydrates & 456 & 455 \\
\hline
\end{tabular}

Table 4.3. Subsystem feature counts obtained for the following genomes: PAO1 from GenBank, the multispecies bench culture. The rows in grey represent subsystems in which all of the gene counts are the same.

Further analysis of the subsystems was focused on the virulence, disease, and defence (VDD) category for interest in potential antibiotic resistance mechanisms. From the RAST server analysis it was possible to obtain a breakdown of the various subsystems present in these categories. There was evidence for a number of efflux pumps present in the bench culture that can confer resistance to all types of drugs, antibiotics, and antimicrobials with 42 genes annotated in various multidrug resistance efflux pump categories (Table 4.4). The PAO1 genome contained 1 less annotation in the multidrug resistance efflux pump category, though all of the functional genes were present. The only functional difference in the VDD category between PAO1 and the bench culture was in the Mycobacterium virulence operon involved in protein 
synthesis (SSU Ribosomal proteins) (6 genes) annotated in the PAO1 genome but not in the bench culture.

\begin{tabular}{|c|c|}
\hline \multicolumn{2}{|c|}{ Virulence, Disease, and Defence subsystem categories and feature counts in $P$. aeruginosa bench culture } \\
\hline \multirow{15}{*}{$\begin{array}{l}\text { Resistance to Antibiotics and } \\
\text { Toxic Compounds }\end{array}$} & Lysozyme inhibitors (3) \\
\hline & Multiple Antibiotic Resistance MAR locus (1) \\
\hline & MexC-MexD-OprJ Multidrug Efflux System (2) \\
\hline & Copper homeostasis (17) \\
\hline & Cobalt-zinc-cadmium resistance (32) \\
\hline & Resistance to Vancomycin (1) \\
\hline & $\begin{array}{l}\text { Multidrug Resistance, Tripartite Systems Found in Gram Negative Bacteria } \\
\text { (14) }\end{array}$ \\
\hline & Resistance to fluoroquinolones (4) \\
\hline & Arsenic resistance (5) \\
\hline & Copper homeostasis: copper tolerance (2) \\
\hline & Fosfomycin resistance (1) \\
\hline & Beta-lactamase (8) \\
\hline & Resistance to chromium compounds (1) \\
\hline & Multidrug Resistance Efflux Pumps (21) \\
\hline & MexE-MexF-OprN Multidrug Efflux System (4) \\
\hline \multirow{2}{*}{$\begin{array}{l}\text { Bacteriocins, ribosomally } \\
\text { synthesized antibacterial } \\
\text { peptides }\end{array}$} & Tolerance to colicin E2 (5) \\
\hline & Colicin V and Bacteriocin Production Cluster (9) \\
\hline \multirow{3}{*}{$\begin{array}{l}\text { Invasion and Intracellular } \\
\text { Resistance }\end{array}$} & Mycobacterium virulence operon involved in DNA transcription (2) \\
\hline & $\begin{array}{l}\text { Mycobacterium virulence operon possibly involved in quinolinate } \\
\text { biosynthesis (3) }\end{array}$ \\
\hline & $\begin{array}{l}\text { Mycobacterium virulence operon involved in protein synthesis (LSU } \\
\text { ribosomal proteins) (3) }\end{array}$ \\
\hline
\end{tabular}

Table 4.4. The subsystem categories found under Virulence, Disease, and Defense following genome annotations of the PAO1 bench culture on the RAST server.

\subsubsection{Metagenome analysis}

All the genome sequences were submitted to the mg-RAST server which annotates metagenomes and identifies multiple bacterial genera present in a sample. The results of the bacterial identities at the genus level from mg-RAST are listed below (Fig. 4.1 and 4.2). Both planktonic cultures that were identified as $P$. aeruginosa using PCR with the $16 \mathrm{~S}$ universal primers U341 and U378 were shown to be $97 \%$ (freezer culture) and 98\% (bench culture) Pseudomonas from the genome sequencing results (Fig. 4.1 A and Fig. 4.2 A, respectively). The percentages given are the total of all sequences, of which $98-99 \%$ is considered high, because some sequences may be too short and can be misidentified. The freezer culture contained 1.1\% Stenotrophomonas, where as the 
agar plate derived culture contained 0.1\% Stenotrophomonas DNA (Fig. 4.1A and Fig. 4.2A, respectively). The bench culture, as previously described, was taken as our pure culture of $P$. aeruginosa, for determination of intrinsic resistance for a relatively pure culture. The freezer culture that was used as inoculum for the remaining biofilm experiments showed an increase in the presence of Stenotrophomonas in the population once in biofilm; the whole biofilm DNA distribution was $70.3 \%$ Pseudomonas and 22.4\% Stenotrophomonas (Fig. 4.1B). The preexposure effluent from another $0.3 \mathrm{~g} / \mathrm{L}$ TSB grown biofilm produced a similar bacterial genus distribution as the whole-biofilm metagenomic DNA; with Pseudomonas and Stenotrophomonas making up $70.2 \%$ and $21.9 \%$ of the bacterial DNA, respectively (Fig. 4.1C). Following the antibiotic exposure and subsequent metabolic recovery back to steady state levels, the biofilm effluent collected and sent for sequencing shifted from a Pseudomonas dominated community to a Stenotrophomonas dominated community with a $65.5 \%$ Stenotrophomonas and $18.1 \%$ Pseudomonas (Fig. 4.1D).

Interestingly, the bacterial community for the $1 \mathrm{mM}$ citrate grown biofilm effluent shows a strong dominance by Pseudomonas, with both pre and post exposure effluent communities dominated by Pseudomonas at 98.1\% and 97.2\%, respectively (Fig. 4.2).

In addition to Stenotrophomonas and Pseudomonas, 16S genes that belonged to other bacterial isolates were identified in the cultures at the genus level including: Xanthomonas $(0.2 \%$ of the planktonic freezer culture; $3.5 \%$ of the biofilm; $4.5 \%$ prexposure effluent and $10 \%$ post exposure effluent in $0.3 \mathrm{~g} / \mathrm{L}$ TSB medium), Microvirus (between $0.3-0.5 \%$ of all cultures), Burkholderia (0.2-0.5\% of $0.3 \mathrm{~g} / \mathrm{L}$ biofilm and effluent samples), Xella $(0.4 \%$ of biofilm and $1 \%$ post exposure biofilm effluent in $0.3 \mathrm{~g} / \mathrm{L}$ TSB medium), Inovirus ( $0.3 \%$ and $0.7 \%$ of pre and post exposure effluent in $1 \mathrm{mM}$ citrate medium), and unclassified DNA labeled "other" (ranging from $1.2 \%$ to $4.5 \%$ of all cultures) (Fig. 4.1 and 4.2 ). 


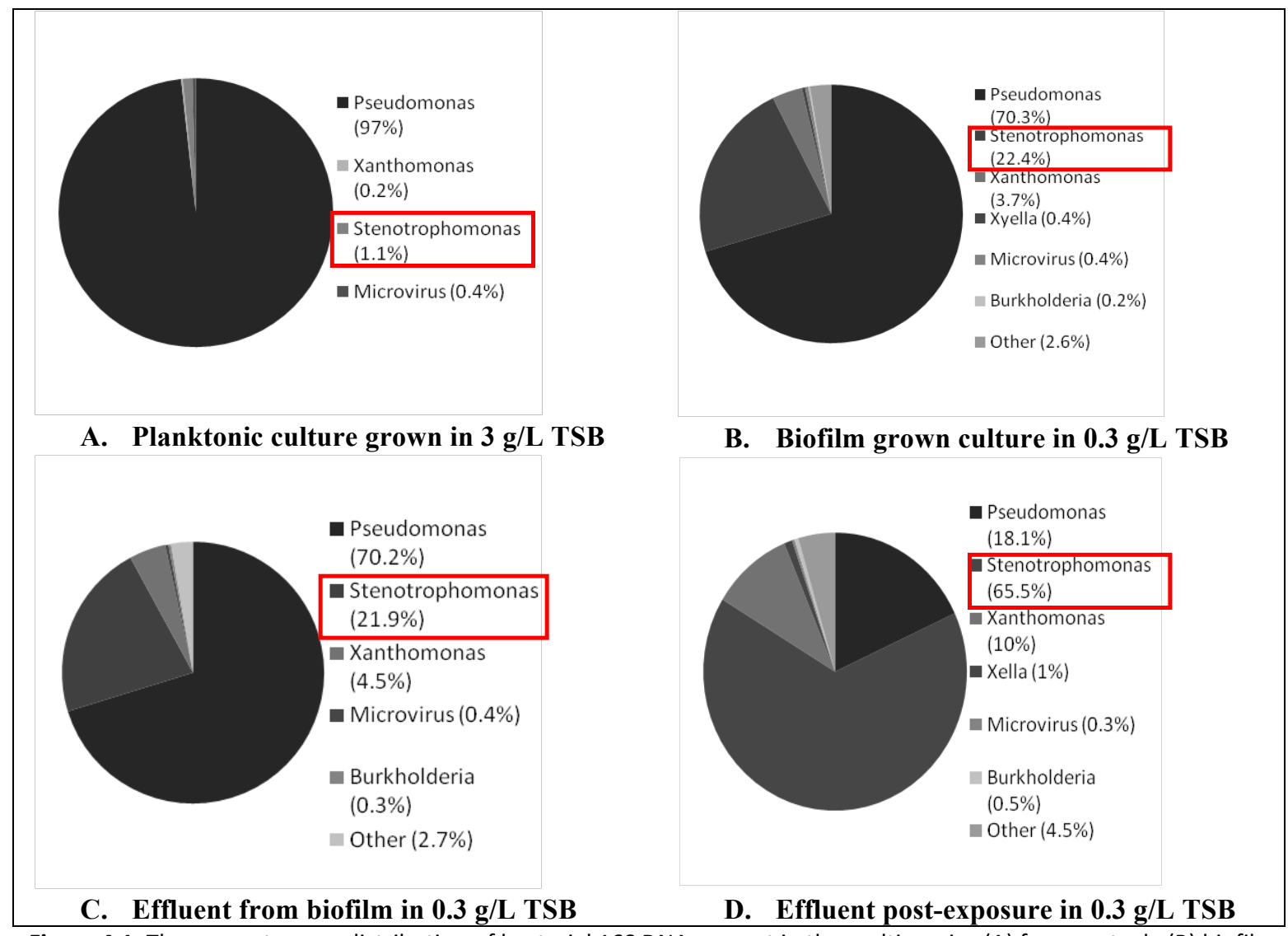

Figure 4.1. The percent genus distribution of bacterial 16S DNA present in the multispecies (A) freezer stock, (B) biofilm, (C) prestreptomycin exposure effluent and, (D) post-streptomycin exposure effluent. The figure demonstrates how the multispecies community shifted from planktonic state to biofilm and following a streptomycin exposure in $0.3 \mathrm{~g} / \mathrm{L} \mathrm{TSB}$ medium. The red boxes demonstrate the shift of Stenotrophomonas in the community.

Species abundance is derived from the number of annotations made at the species level from all the annotation source databases used by mg-RAST (Meyer et al., 2008). The most common Pseudomonas strain in each of the cultures was $P$. aeruginosa (PAO1) and the most common Stenotrophomonas strain annotated was S. maltophilia (R551-3). 


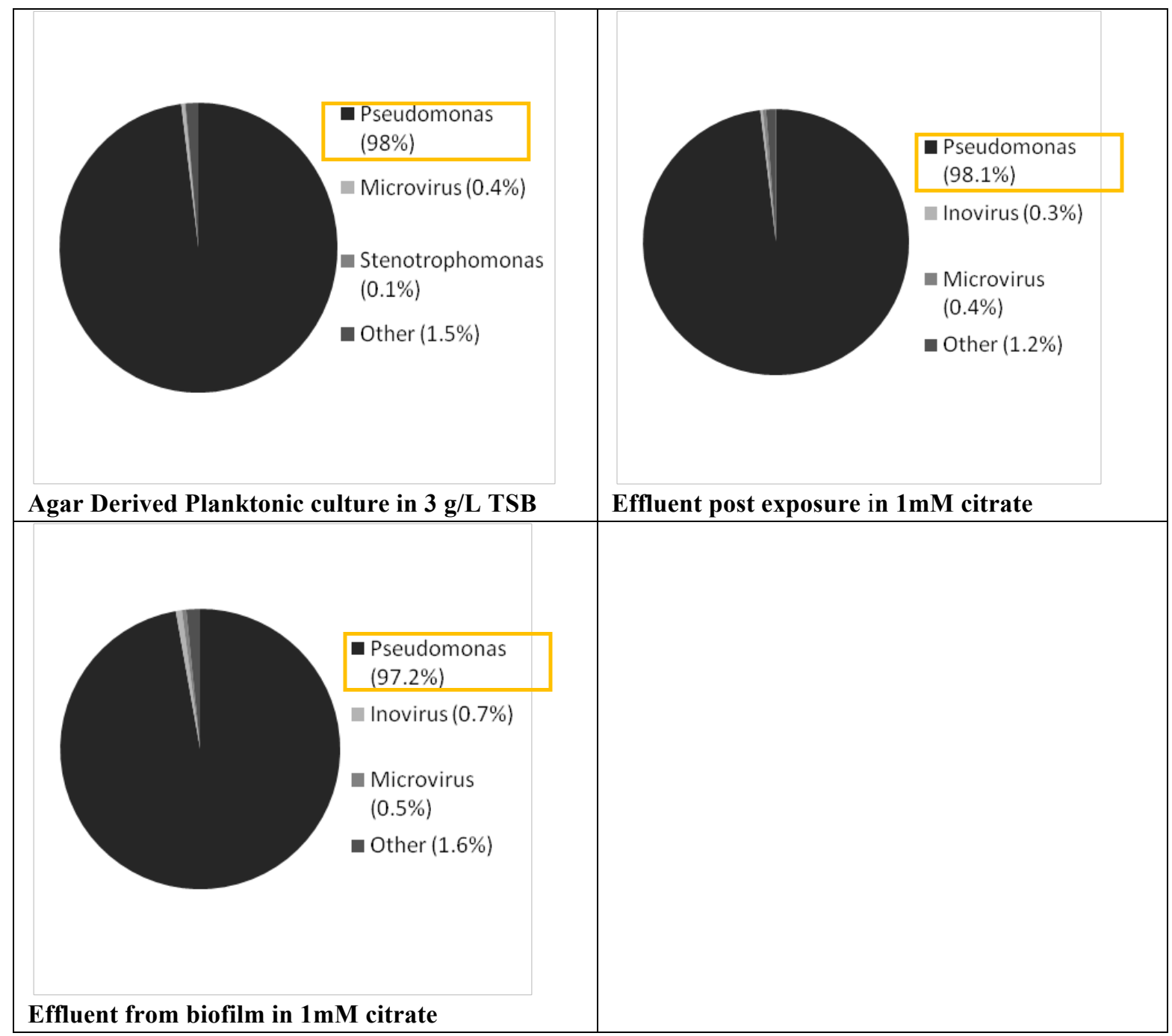

Figure 4.2. The percent genus distribution of bacterial $16 \mathrm{~S}$ DNA present in the multispecies (A) bench cultures grown in $0.3 \mathrm{~g} / \mathrm{L}$ TSB, (B) effluent collected from pre-streptomycin exposure in $1 \mathrm{mM}$ citrate medium and, (C) effluent collected from prestreptomycin exposure in $1 \mathrm{mM}$ citrate medium. The orange boxes highlight the fact that each of these cultures was largely dominated by Pseudomonas.

The mg-RAST server for metagenome annotation allowed for functional based comparison (among other features) of the number of unique sequence reads (called hits) for each of the metagenomes uploaded to their server. By comparing the unique gene sequences among the samples, found in each subsystem category, we can determine what sort of genetic changes are occurring from planktonic to biofilm cultures or pre- and post-antibiotic exposure when the communities shift. Table 4.5 shows that the groups dominated by Stenotrophomonas (all the TSB grown cultures: column 2-5) contain more sequence reads in the gene category for VDD. Moreover, the biofilm metagenome analysis had far more hits in each subsystem category compared to all of the other cultures sequenced, in some instances more than doubling in the number of unique sequence reads. It is important to note however, that each of the genomes annotated are draft genomes, due to incomplete coverage of the genomes from the 
sequencing.

\begin{tabular}{|c|c|c|c|c|c|c|}
\hline \multirow[b]{2}{*}{ Subsystem } & \multicolumn{6}{|c|}{ Number of genes identified } \\
\hline & $\begin{array}{l}\text { Planktonic DNA } \\
\text { from Freezer } \\
\text { Culture in TSB }\end{array}$ & $\begin{array}{l}\text { Biofilm } \\
\text { in TSB }\end{array}$ & $\begin{array}{l}\text { Pre- } \\
\text { exposure } \\
\text { effluent } \\
\text { (TSB) }\end{array}$ & $\begin{array}{l}\text { Post- } \\
\text { exposure } \\
\text { effluent } \\
\text { (TSB) }\end{array}$ & $\begin{array}{l}\text { Pre- } \\
\text { exposure } \\
\text { effluent } \\
\text { (citrate) }\end{array}$ & $\begin{array}{l}\text { Post- } \\
\text { exposure } \\
\text { effluent } \\
\text { (citrate) }\end{array}$ \\
\hline Amino Acids and Derivatives & 1808 & 2660 & 1646 & 1606 & 1143 & 1145 \\
\hline Carbohydrates & 1528 & 2301 & 1467 & 1455 & 898 & 888 \\
\hline Cell Division and Cell Cycle & 201 & 323 & 206 & 209 & 112 & 111 \\
\hline Cell Wall and Capsule & 723 & 1159 & 803 & 794 & 434 & 444 \\
\hline Clustering-based subsystems & 2699 & 4034 & 2752 & 2744 & 1599 & 1587 \\
\hline $\begin{array}{l}\text { Cofactors, Vitamins, Prosthetic } \\
\text { Groups, Pigments }\end{array}$ & 1158 & 1765 & 1145 & 1141 & 705 & 699 \\
\hline DNA Metabolism & 482 & 823 & 492 & 500 & 262 & 260 \\
\hline Dormancy and Sporulation & 28 & 37 & 24 & 21 & 13 & 13 \\
\hline Fatty Acids, Lipids, and Isoprenoids & 580 & 845 & 577 & 564 & 349 & 357 \\
\hline Iron acquisition and metabolism & 536 & 709 & 524 & 505 & 321 & 320 \\
\hline Membrane Transport & 1026 & 1437 & 1000 & 970 & 619 & 617 \\
\hline Metabolism of Aromatic Compounds & 402 & 504 & 328 & 323 & 271 & 276 \\
\hline Miscellaneous & 1533 & 2362 & 1571 & 1596 & 929 & 901 \\
\hline Motility and Chemotaxis & 323 & 488 & 351 & 352 & 188 & 194 \\
\hline Nitrogen Metabolism & 259 & 327 & 196 & 192 & 177 & 170 \\
\hline Nucleosides and Nucleotides & 458 & 676 & 422 & 418 & 266 & 250 \\
\hline $\begin{array}{l}\text { Phages, Prophages, Transposable } \\
\text { elements, Plasmids }\end{array}$ & 300 & 458 & 332 & 327 & 171 & 169 \\
\hline Phosphorus Metabolism & 212 & 338 & 207 & 197 & 133 & 123 \\
\hline Photosynthesis & 8 & 17 & 10 & 10 & 3 & 5 \\
\hline Potassium metabolism & 97 & 142 & 101 & 98 & 63 & 64 \\
\hline Protein Metabolism & 1095 & 1758 & 1125 & 1121 & 627 & 648 \\
\hline RNA Metabolism & 657 & 1030 & 680 & 692 & 380 & 374 \\
\hline Regulation and Cell signaling & 461 & 677 & 506 & 500 & 306 & 317 \\
\hline Respiration & 547 & 824 & 519 & 525 & 300 & 299 \\
\hline Secondary Metabolism & 88 & 120 & 87 & 86 & 74 & 71 \\
\hline Stress Response & 637 & 967 & 649 & 663 & 402 & 392 \\
\hline Sulfur Metabolism & 284 & 369 & 240 & 243 & 191 & 191 \\
\hline Virulence, Disease and Defense & 688 & 1109 & 740 & 748 & 384 & 388 \\
\hline
\end{tabular}

Table 4.5. The number of hits (unique sequence reads) from the subsystem categories of functional genes for each of the culture-derived genomic DNA sequences annotated using the mg-RAST server.

In Table 4.6, all of the annotated genes relating to aminoglycoside resistance for each of the samples submitted for metagenomic analysis are listed. All of the samples (numbered 1-7) contained genes annotated with aminoglycoside 3'-phosphotransferase type-IIb function. Samples 2-5 all contained genes that function as aminoglycoside response regulators. Sample 3 uniquely contained an aminoglycoside-6"phosphotransferase. Sample 4 uniquely had an 
aminoglycoside/hydroxyurea antibiotic resistance kinase gene annotated. Lastly, sample 7 contained hits for modifying enzymes in the acetyl-transferase (AAC) class. The two samples containing the largest number of functional genes were samples 3 and 7, the whole-biofilm and the post-exposure effluent from the $1 \mathrm{mM}$ citrate grown biofilms.

\begin{tabular}{|c|c|c|c|}
\hline Metagenome & Source & Function & $\begin{array}{l}\text { Species used for } \\
\text { annotation }\end{array}$ \\
\hline 1 & GenBank & aminoglycoside 3'-phosphotransferase type IIb & P. aeruginosa \\
\hline 1 & GenBank & aminoglycoside phosphotransferase & S. maltophilia \\
\hline 2 & GenBank & aminoglycoside 3'-phosphotransferase type IIb & P. aeruginosa $\mathrm{PAO} 1$ \\
\hline 2 & GenBank & aminoglycoside phosphotransferase & *EC:2.7.1.95 \\
\hline 2 & GenBank & aminoglycoside response regulator & P. aeruginosa \\
\hline 3 & GenBank & aminoglycoside 3'-phosphotransferase & S. maltophilia \\
\hline 3 & GenBank & aminoglycoside 3'-phosphotransferase type IIb & P. aeruginosa $\mathrm{PAO} 1$ \\
\hline 3 & GenBank & aminoglycoside 3-phosphotransferase type IIb & P. aeruginosa UCBPP-PA14 \\
\hline 3 & GenBank & aminoglycoside 6"-phosphotransferase & $* \mathrm{EC}: 2.7 .1 .72$ \\
\hline 3 & GenBank & aminoglycoside response regulator & P. aeruginosa $\mathrm{PAO} 1$ \\
\hline 4 & GenBank & aminoglycoside 3'-phosphotransferase & S. maltophilia \\
\hline 4 & GenBank & aminoglycoside 3'-phosphotransferase type IIb & P. aeruginosa $\mathrm{PAO} 1$ \\
\hline 4 & GenBank & aminoglycoside phosphotransferase & S. maltophilia \\
\hline 4 & GenBank & aminoglycoside response regulator & P. aeruginosa $\mathrm{PAO} 1$ \\
\hline 4 & GenBank & $\begin{array}{l}\text { aminoglycoside/hydroxyurea antibiotic } \\
\text { resistance kinase }\end{array}$ & S. maltophilia \\
\hline 5 & GenBank & aminoglycoside 3'-phosphotransferase & S. maltophilia \\
\hline 5 & GenBank & aminoglycoside 3'-phosphotransferase type IIb & P. aeruginosa $\mathrm{PAO} 1$ \\
\hline 5 & GenBank & aminoglycoside response regulator & P. aeruginosa \\
\hline 6 & GenBank & aminoglycoside 3'-phosphotransferase type IIb & $P$. aeruginosa $\mathrm{PAO} 1$ \\
\hline 6 & GenBank & aminoglycoside phosphotransferase & *EC:2.7.1.95 \\
\hline 6 & GenBank & aminoglycoside response regulator & P. aeruginosa \\
\hline 7 & GenBank & $\begin{array}{l}\text { aminoglycoside-(3)-acetyltransferase AacC-A1 } \\
\text { or AacC1 or AAC-(3)-la }\end{array}$ & $* \mathrm{EC}: 2.3 .1 .60 *$ \\
\hline 7 & GenBank & aminoglycoside 3'-phosphotransferase type IIb & P. aeruginosa $\mathrm{PAO} 1$ \\
\hline 7 & GenBank & aminoglycoside N (3')-acetyltransferase I & Acinetobacter baumannii \\
\hline 7 & GenBank & aminoglycoside phosphotransferase & S. maltophilia \\
\hline 7 & GenBank & aminoglycoside response regulator & P. aeruginosa \\
\hline
\end{tabular}

Table 4.6. Annotated aminoglycoside resistance related genes from metagenomic analysis. Each culture is present and numbered 1-7 as follows: 1) Planktonic Freezer stock in $3 \mathrm{~g} / \mathrm{L} \mathrm{TSB}, 2)$ Planktonic bench culture in $3 \mathrm{~g} / \mathrm{L} \mathrm{TSB}, 3$ ) Biofilm grown in $0.3 \mathrm{~g} / \mathrm{L} \mathrm{TSB}, 4)$ Pre-exposure biofilm effluent in $0.3 \mathrm{~g} / \mathrm{L} \mathrm{TSB}, 5)$ Post-exposure biofilm effluent in $0.3 \mathrm{~g} / \mathrm{L}$ TSB, 6) Pre-exposure biofilm effluent in $1 \mathrm{mM}$ citrate medium, and 7) Post-exposure biofilm effluent in $1 \mathrm{mM}$ citrate grown medium. All functional annotations were sourced from Genbank, and the genes were categorized according to function. The abundance signifies how many times that particular gene was found, the average \% identity represents how closely the annotated genes from the samples submitted match the Genbank gene sequences, and the number of hits represents the total unique sequence reads for the gene identified. *The EC numbers from the KEGG database were given for the genes that were annotated from multiple species or were from plasmids (Kanehisa and Goto, 2000). 


\subsection{Discussion}

PCR primers (U341F/ U758R) that span and amplify a 418 bp segment of the 16S rRNA gene were used in this study to verify bacterial strain identity. The amplified and sequenced gene segments were compared with NCBI's database using BLAST that resulted in a $>98 \%$ similarity for the strains tested as: P. aeruginosa, Sphingobacterium, P. putida, E. absuriae, and an Enterobacter sp. One drawback to PCR, however, is that some gene sequences may be preferentially amplified over others - potentially shielding the presence of low copy number templates. Factors that affect PCR amplification include: template DNA concentration, number of cycles and inhibition of template binding from DNA in flanking regions to name a few (Hansen et al., 1998). For the multispecies culture containing $P$. aeruginosa it is likely that the PCR missed the presence of the $16 \mathrm{~S}$ gene from other species. The amplification was performed on genomic DNA that was extracted from planktonic cultures which likely had lower copy numbers of the $16 \mathrm{~S}$ gene for species other than P. aeruginosa. Even if multiple $16 \mathrm{~S}$ genes were amplified, the Sanger reads would have resulted in indistinguishable results due to multiple overlapping sequences (Salipante et al., 2013). This helps to explain several failed attempts at achieving a proper $16 \mathrm{~S}$ read on the multispecies culture PCR results.

Survival to exposures of high doses of streptomycin was observed and described in the earlier chapters in the 3 Gram-negative environmental isolates and multispecies cultures from the bench and freezer stocks, and uncovering potential mechanisms for resistance in these cultures was of interest. Initially, each of the cultures was tested for the presence of aminoglycoside modifying enzymes. $P$. putida was the only isolate to show a positive result for the amplification of an aminoglycoside modifying enzyme, specifically aph (3"). PCR amplification with the streptomycin modifying enzyme primers of the multispecies cultures did not produce amplicons. However, genomic analysis revealed the presence of various aminoglycoside modifying enzymes annotated from the metagenomes (Table 4.6). Genome sequencing on the multispecies cultures helped create a more complete picture of their genetic potential.

Genetic analysis is useful for environmental studies where culturing techniques are not always possible (Torsvik and Øvreås). Even in laboratory settings not all members in a community may be evident due to culturing conditions that favour one community member over another. However, conditions may arise where the less dominant community members are given the 
opportunity to increase in numbers (if they have been able to persist). Metagenomic analysis provides information on the functional genes present in a community, community composition and community dynamics (Tringe and Rubin, 2005). In this study, each of the cultures sent for whole-genome sequencing showed the presence of multiple bacteria or viruses in the population. When cultures were grown under Pseudomonas specific conditions (in the citrate defined medium) the mixture co-cultures were very low (Fig. 4.2B, C). The defined citrate medium was designed specifically for the growth and minimal nutritional needs of $P$. aeruginos $a$ in order to reduce the likelihood of growth of other species (Clark and Maaløe, 1967). Though relatively pure, the bench culture streaked from single colonies revealed that $0.1 \%$ of the DNA sequenced belonged to Stenotrophomonas (Fig. 4.2A). Interestingly, repeated streak plating and dilutions plated from biofilm effluent of the freezer culture and bench culture never revealed differences in colony morphology.

Metagenomic analysis revealed changes in community composition of the cultures inoculated from the multispecies biofilm freezer stocks when grown in planktonic culture compared to biofilm and in the biofilm effluent before and after antibiotic exposure (Figure 4.1). The metagenomic analysis revealed a community population ratio shift from $98 \%$ Pseudomonas and $1.1 \%$ Stenotrophomonas (Fig. 4.1A) to 70\% Pseudomonas and 22\% Stenotrophomonas in whole biofilm extract and a biofilm effluent (Fig. 4.1B and 4.1C, respectively). In the planktonic cultures Stenotrophomonas was only a small part of the bacterial community, but once a biofilm had formed Stenotrophomonas increased its proportion in the population. The increase in the proportion of Stenotrophomonas upon growth in biofilm was likely beneficial for the Pseudomonas present in the cultures, as Stenotrophomonas carries many antimicrobial resistance genes with it which may benefit other genes members within the biofilm. This is demonstrated by a second community ratio shift that was observed following a streptomycin exposure on the biofilms. The effluent collected from the biofilms following a streptomycin exposure had a culture largely dominated by Stenotrophomonas (Fig. 4.1D). It is important to note that there are less expensive methods of detecting multiple species in a sample such as denaturing gradient gel electrophoresis (DGGE) or 16S $2^{\text {nd }}$ generation sequencing (Salipante et al., 2013; Brons and Elsas, 2008). Thus, in order to check sample purity in the future when whole genome analysis is not of interest, these other methods would be more appropriate. 
Determining exactly which bacteria are present in the cultures being tested, especially if their numbers are very low in the community, can be a difficult task. As one study points out, the DNA extraction kits and reagents used to prepare DNA samples for further analysis have widespread DNA contamination. Bacterial DNA commonly found as contaminants in DNA extraction kits include several of the bacteria sequenced in this study such as: Pseudomonas, Stenotrophomonas, Burkholderia, and Xanthomonas (Salter et al., 2014). Thus, it may be likely that several of the bacteria that were identified from metagenomic analysis were not actually present in our samples, but the DNA was present in the isolation kits. One way to circumvent this issue in the future is to include a negative control when sending samples for sequencing (Salter et al., 2014). Unfortunately, the high cost of sequencing presents a noticeable impediment. For the purpose of this study, we are assuming that the presence of Pseudomonas and Stenotrophomonas in the community was a constant because they are present (even if in very low numbers) in each of the cultures and their numbers shift as culture conditions change. This suggests Pseudomonas and Stenotrophomonas DNA is not simply there as a result of contamination in the microbial DNA extraction kits.

The $P$. aeruginosa and $S$. maltophilia strains with the highest number of sequence reads annotated in each of the cultures were PAO1 and R551-3, respectively. P. aeruginosa PAO1 is a prototype strain commonly used in laboratories and its genome has been fully sequenced (Kukavica-Ibrulj et al., 2008). The S. maltophilia strain R551-3 is a plant associated strain isolated from poplar roots whose full genome has been sequenced and has a high degree of sequence similarity to the human-associated pathogen $S$. maltophilia K279a (Taghavi et al., 2009; Alavi et al., 2014; Abbott et al., 2011). Both P. aeruginosa PAO1 and S. maltophilia R551-3 have naturally occurring virulence factors, antimicrobial resistance genes and can grow at $37^{\circ} \mathrm{C}$ which make them comparable to more pathogenic strains of Pseudomonas and Stenotrophomonas (Reimmann et al., 1997; Hancock and Brinkman, 2002; Alavi et al., 2009; Ryan et al., 2009). The co-existence of these bacteria can be mutually beneficial when they are harbouring a variety of resistance genes that can later help protect the community from an array of environmental stressors.

P. aeruginosa is the most common Gram-negative opportunistic pathogen (Crossman et al., 2008), whereas S. maltophilia was found to be the third most common non-enteric Gram- 
negative bacilli from a large scale study that isolated over 200,000 bacteria from 1997-2003 (Sader and Jones, 2005). Both Stenotrophomonas and Pseudomonas are major bacterial constituents present in a variety of chronic wounds (Dowd et al., 2008; Nazik et al., 2007). Studying multispecies biofilms containing the opportunistic pathogens $P$. aeruginos $a$ and $S$. maltophilia is relevant since they are commonly isolated together from sick and injured patients. Both S. maltophilia and P. aeruginosa can be found in the lungs of cystic fibrosis patients (Wu et al., 2013; Gabriel et al., 2004; O’May et al., 2006; Gordon et al., 1988). Bacterial composition in a venous leg ulcer sample by ribosomal amplification and pyrosequencing showed a ratio of 0.58\% Pseudomonas and 11.8\% Stenotrophomonas spp. and while full ribosomal amplification of the ulcer resulted in $15.46 \%$ P. aeruginosa and 7.22\% S. maltophilia (Dowd et al., 2008). Another study found that $P$. aeruginosa was the most common co-pathogen isolated from patients with nosocomial pneumonia with $S$. maltophilia and that S. maltophilia was associated with high mortality rates (Tseng et al., 2009). However, S. maltophilia colonization is not always associated with mortality and disease, but it can act as an indirect pathogen (Ryan et al., 2009). One study demonstrated that S. maltophilia aided in the survival of susceptible strains of P. aeruginosa and Serratia marcescens in the presence of beta-lactam antibiotics (Kataoka et al., 2003). S. maltophilia has been shown to carry a variety of antibiotic resistance genes and to have intrinsic resistance to beta-lactams and aminoglycosides (Ryan et al., 2009). Thus, one can speculate that the presence of $S$. maltophilia may also aid in resistance to certain aminoglycoside antibiotics.

In Table 4.5, the number of unique sequence reads for each of the genomes analyzed demonstrates that there were variations in the different cultures in terms of genetics as well as community composition (seen in Figures 1 and 2). It is not surprising to see increases in the presence of genes with increasing numbers of bacterial isolates in the community and it is relevant when trying to decipher the intrinsic nature of the cultures tested. It is important to note that the unique database sequences (or hits) are derived from the similarity search, which is not the same as the number of reads (Meyer et al., 2008). The number of annotations compared to the number of reads may differ because; (1) multiple predicted protein fragments on contigs can result in more than one annotated gene and/or, (2) sequences may be present in the underlying data sources multiple times and with distinct labels (Meyer et al., 2008). 
Aminoglycoside modifying enzymes are specific to a subset of aminoglycoside antibiotics as a result of structural differences in the chemicals and the enzyme active sites (Shaw et al., 1993). Our experiments were performed with streptomycin and gentamicin antibiotics. Therefore, it is important to know which antibiotics the enzymes annotated from the genomes will modify. A summary of all the aminoglycoside enzymes annotated from our cultures can be found in Table 4.6. The aminoglycoside modifying enzymes enzymatically inactivate aminoglycosides through hydroxyl or amino-groups of the 2-deoxystreptamine nucleus or the sugar moieties (Ramirez and Tomalsky, 2010). The enzymes are grouped into 3 main categories for the type of modification used (adenylation, acetylation, and phosphorylation) (Shaw et al., 1993). The aminoglycoside phosphotransferases (APH) can confer resistance to streptomycin or gentamicin. Based on the metagenome annotations all of the cultures sequenced contained the gene aph coding for APH (3)-IIb enzyme. The aph (6") gene (also known as strB) was only found in the biofilm metagenome (Table 4.6) (Scholz et al., 1989). The APH (3)-IIb enzyme can modify aminoglycosides such as gentamicin (not streptomycin) and the APH (6) group of enzymes only modifies streptomycin antibiotics (Shaw et al., 1993; Chiou and Jones, 1995). Aminoglycoside acetyltransferases (AAC) that confer resistance to gentamicin were annotated in effluent collected from a citrate grown biofilm exposed to streptomycin (Table 4.6). Aminoglycoside acetyltransferases' do not produce resistance to the streptomycin; however, AACs can confer resistance to gentamicin depending on the antibiotic site that they modify. In particular, the AAC enzyme AAC (3)-Ia (also known as AacC-A1 or AacC) can modify gentamicin (Levings et al., 2005; Shaw et al., 1993). Another aminoglycoside resistance gene that was present in each of the cultures sequenced (except the planktonic derived freezer stock) was the aminoglycoside response regulator (the arr gene). The arr gene was discovered in P. aeruginosa and shown to be required for tobramycin induced biofilm formation; streptomycin and gentamicin induced biofilm formations were at $66 \%$ and $25 \%$ of that by tobramycin (Hoffman et al., 2005). The last gene related to aminoglycoside found in the annotated genomes was aminoglycoside/hydroxyurea antibiotic resistance kinase gene that confers resistance to streptomycin and spectinomycin (Bai et al., 2013). The aminoglycoside/hydroxyurea antibiotic resistance kinase gene was found in TSB grown pre-exposure biofilm effluent, however, was not observed in the post-exposure effluent sample (Table 4.6). The planktonic cultures contained the least number of aminoglycoside related resistance genes, which may be due to the lower copy 
number of these genes in the population that the genome sequencing failed to sequence. The unexposed whole-biofilm extracted DNA and the streptomycin exposed biofilm effluent grown in citrate media had the largest number of genes relating to the streptomycin resistance annotated.

Only two cultures were monitored pre and post streptomycin exposure; the effluent cultures grown in TSB and citrate medium. In TSB medium, pre-exposure effluent contained genes with the potential to aid in streptomycin resistance (aminoglycoside/hydroxyurea antibiotic resistance kinase gene) (Table 4.6). However, the post-exposure effluent did not show the presence of this gene, indicating that its presence may not have been pertinent to biofilm survival following the streptomycin exposure. In the citrate grown biofilms the pre-exposure effluent contained genes for aminoglycoside modifying enzymes, but none that can modify streptomycin. The postexposure effluent from citrate grown biofilms contained genes for AAC enzymes capable of modifying streptomycin (Table 4.6). Therefore, there may have been selection of bacterial cells containing the resistance genes following the streptomycin exposure allowing these genes to increase in copy number. Whether the cultures were expressing aminoglycoside modifying enzymes or resistance genes is not known. Metagenomics can give information on the gene variability in the communities tested; nonetheless, methods such as quantitative PCR would be needed to determine levels of gene expression (Frias-Lopez et al., 2008).

\subsection{Conclusion}

Full genome sequencing and metagenomic analysis has provided insight into the intrinsic potential and community composition of the multispecies biofilms tested throughout this dissertation. The low levels of $S$. maltophilia maintained within the planktonic and bench cultures are of importance because harbouring of DNA can play a large role when selection pressure for certain genes from environmental stressors arise. Future work will include monitoring changes in community composition with quantitative-PCR and monitoring community member activity through reverse transcriptase-qPCR. The overall goal of future work will be to determine the differences between pure and dual-cultures that will allow us to understand how S. maltophilia can be beneficial to P. aeruginosa. 


\section{Summary}

Bacterial biofilms are a major concern to the healthcare community as they result in incurable diseases in immunocompromised patients, those with implanted medical devices or open wounds. Biofilms are difficult to treat with conventional antibiotic regimes due to growing levels of resistance and the many advantages incurred from the biofilm mode of growth. However, antibiotics remain the main method used to treat bacterial infections. Research in antibiotic resistance in biofilms can help better utilize current antibiotics, determine ways to increase biofilm antibiotic susceptibility, prevent future antibiotic resistance outbreaks, and improve use of future antibiotics. Monitoring biofilm behaviour with real-time $\mathrm{CO}_{2}$ measurements in CEMS allowed for a unique look at how whole-biofilms, both multispecies and pure cultures, responded to antibiotics in various environmental conditions.

Analysis of whole-biofilm behaviour demonstrated that it is not always possible to infer biofilm response to antibiotics in the presence of different carbon sources and nutrient conditions based on established planktonic cell studies. The same is true for biofilm response to aminoglycosides at different $\mathrm{pH}$ conditions. This is important because all patient wound-related biofilms are affected by varying nutrient and $\mathrm{pH}$ conditions.

Understanding how nutrients and $\mathrm{pH}$ affect individual bacteria in pure cultures and in multispecies communities can help devise more effective ways of administering antibiotics. Some bacteria, such as $P$. aeruginosa species, have highly diverse nutrient profiles enabling them to thrive in a variety of environments. Metabolism of different nutrients changes gene upregulation, cell composition and structure, and bacterial response to antimicrobials.

Experiments in this study revealed that added carbon decreased susceptibility to streptomycin on young multispecies biofilms. Experiments performed on P. aeruginosa monocultures with and without the ability to efflux streptomycin antibiotics demonstrated a potential link to antibiotic efflux and survival of the monocultures. However, the mechanism of survival from multispecies cultures may differ depending on how nutrient changes affect each community member. Future work should take into account the behaviour of the individual bacteria present in the multispecies cultures and how they respond to each other and the nutrient changes. 
It has been demonstrated previously, and in this dissertation, that aminoglycoside susceptibility is highly dependent on $\mathrm{pH}$. However, the results from this work reveal differences in biofilms response to $\mathrm{pH}$ compared to planktonic cells. In this study, aminoglycoside exposures do not show an increase in biofilm susceptibility to the antibiotics when increasing the $\mathrm{pH}$ of the antibiotic medium from acidic to basic $\mathrm{pH}$, which has been shown in planktonic systems. An interesting observation that should be further explored is the biofilm's ability to adjust the $\mathrm{pH}$ of the influent medium from acidic and basic $\mathrm{pH}$ back to neutral. Future studies will be performed to separate the effects of $\mathrm{pH}$ versus the antibiotic to determine if there was indeed a dual stress occurring on the biofilm during these exposures that affected aminoglycoside susceptibility. In addition, experiments with a $P$. aeruginosa monoculture deficient in antibiotic efflux will be performed to help infer whether antibiotic efflux or antibiotic uptake is more affected at the different $\mathrm{pH}$ tested.

To further understand the multispecies cultures that were used in this study full genome sequencing and metagenomic analysis were performed. Metagenomic analysis on the multispecies biofilms revealed that the cultures were dominated by the Pseudomonas aeruginosa and Stenotrophomonas maltophilia strains. The dominant strain in planktonic cultures was $P$. aeruginosa. However, in complex growth medium S. maltophilia increased in proportion in the population upon biofilm development and became the dominant species following recovery of a high dose streptomycin exposure. Metagenomic analysis uncovered community composition and antibiotic resistance genes that can play a role in the survival of the multispecies biofilms to aminoglycoside antibiotics.

Biofilm behaviour and susceptibility to antibiotics is complex. There are many biofilm-specific physiological characteristics that prevent antibiotics from reaching their targets in addition to antibiotic resistance that can occur from genetically related mechanisms. The complexity of biofilm research increases when studying multispecies biofilms co-inhabiting chronic wounds and other environments. Therefore, exploring how biofilm genetics and dynamics are linked to antibiotic resistance under different environmental conditions will be essential to effectively treat biofilm infections. Future work will compare pure cultures of S. maltophilia and P. aeruginosa in their survival to streptomycin in various media types to determine how each species survives high-dose antibiotic exposures when provided with different carbon sources. In addition, cultures 
will be made with different levels of each strain (e.g. $1 \%$ S. maltophilia to $99 \%$ P. aeruginosa, and 50:50 mxtures to determine if there is a threshold S. maltophilia concentration that needs to be present in order to have survival to streptomycin in $0.3 \mathrm{~g} / 1 \mathrm{TSB}$ medium. 


\section{References}

1. Abbott, I. J., Slavin, M. A., Turnidge, J. D., Thursky, K. A. \& Worth, L. J.

Stenotrophomonas maltophilia: emerging disease patterns and challenges for treatment. Expert Rev. Anti. Infect. Ther. 9, 471-88 (2011).

2. Abràmoff, M. D., Magalhães, P. J. \& Ram, S. J. Image processing with ImageJ Part II. Biophotonics Int. 11, 36-43 (2004).

3. Alavi, P., Starcher, M. R., Thallinger, G. G., Zachow, C., Müller, H. \& Berg, G.Stenotrophomonas comparative genomics reveals genes and functions that differentiate beneficial and pathogenic bacteria. BMC Genomics 15, 482 (2014).

4. Allesen-Holm, M., Barken, K. B., Yang, L., Klausen, M., Webb, J. S., Kjelleberg, S., Molin, S., Givskov, M., \& Tolker-Nielsen, T. A characterization of DNA release in Pseudomonas aeruginosa cultures and biofilms. Mol. Microbiol. 59, 1114-1128 (2006).

5. Allison, K. R., Brynildsen, M. P. \& Collins, J. J. Metabolite-enabled eradication of bacterial persisters by aminoglycosides. Nature 473, 216-220 (2011).

6. Aminov, R. I. A brief history of the antibiotic era: lessons learned and challenges for the future. Front. Microbiol. 1, 134 (2010).

7. Andrews, J. M. Determination of minimum inhibitory concentrations. J. Antimicrob. Chemother. 48, 5-16 (2001).

8. Araoka, H., Baba, M. \& Yoneyama, A. Risk factors for mortality among patients with Stenotrophomonas maltophilia bacteremia in Tokyo, Japan, 1996-2009. Eur. J. Clin. Microbiol. Infect. Dis. 29, 605-608 (2010).

9. Ashby, M. J., Neale, J. E., Knott, S. J. \& Critchley, I. A. Effect of antibiotics on nongrowing planktonic cells and biofilms of Escherichia coli. J. Antimicrob. Chemother. 33, 443-452 (1994).

10. Aziz, R. K., Bartels, D., Best, A. A., DeJongh, M., Disz, T., Edwards, R. A... \& Zagnitko, O. The RAST Server: rapid annotations using subsystems technology. BMC Genomics 9, 75 (2008).

11. Bai, J., Liu, Q., Yang, Y., Wang, J., Yang, Y., Li, J., Li, P., Li, X., Ying, J., Ren, P., Yang, L., Ni, L., Wu, J., Qiyu, B. \& Zhou, T. Insights into the evolution of gene organization and multidrug resistance from Klebsiella pneumoniae plasmid pKF3-140. Gene 519, 60-66 (2013). 
12. Baltz, R. H. Renaissance in antibacterial discovery from actinomycetes. Curr. Opin. Pharmacol. 8, 557-563 (2008).

13. Beceiro, A., Tomás, M. \& Bou, G. Antimicrobial resistance and virulence: a successful or deleterious association in the bacterial world? Clin. Microbiol. Rev. 26, 185-230 (2013).

14. Bester, E., Kroukamp, O., Wolfaardt, G. M., Boonzaaier, L. \& Liss, S. N. Metabolic differentiation in biofilms as indicated by carbon dioxide production rates. Appl. Environ. Microbiol. 76, 1189-1197 (2010).

15. Bezanson, G. S., MacInnis, R., Potter, G. \& Hughes, T. Presence and potential for horizontal transfer of antibiotic resistance in oxidase-positive bacteria populating raw salad vegetables. Int. J. Food Microbiol. 127, 37-42 (2008).

16. Booth, I. R. Regulation of cytoplasmic $\mathrm{pH}$ in bacteria. Microbiol. Rev. 49, 359-378 (1985).

17. Borriello, G., Richards, L., Ehrlich, G. D., Stewart, S. \& Stewart, P. S. Arginine or Nitrate Enhances Antibiotic Susceptibility of Pseudomonas aeruginosa in Biofilms Arginine or Nitrate Enhances Antibiotic Susceptibility of Pseudomonas aeruginosa in Biofilms. 1-4 (2006).

18. Breeuwer, P. \& Abee, T. Assessment of the membrane potential, intracellular $\mathrm{pH}$ and respiration of bacteria employing fluorescence techniques. Mol. Microb. Ecol. Manual, Second Ed. 1563-1580 (2004).

19. Brittain, H. G. Profiles of Drug Substances, Excipients and Related Methodology. Analytical Profiles of Drug Substances and Excipients 29 (2002): 1-5.

20. Brons, J. K. \& van Elsas, J. D. Analysis of bacterial communities in soil by use of denaturing gradient gel electrophoresis and clone libraries, as influenced by different reverse primers. Appl. Environ. Microbiol. 74, 2717-27 (2008).

21. Brooke, J. S. Stenotrophomonas maltophilia: An emerging global opportunistic pathogen. Clin. Microbiol. Rev. 25, 2-41 (2012).

22. Brooun, A., Liu, S. \& Lewis, K. I. M. A dose-response study of antibiotic resistance in Pseudomonas aeruginosa biofilms. Antimicrob. Agents Chemother. 44, 640-646 (2000).

23. Brown, M. R. W., Collier, P. J. \& Gilbert, P. Influence of growth rate on susceptibility to antimicrobial agents : Modification of the cell envelope and batch and continuous culture studies. 34, 1623-1628 (1990). 
24. Brown, M. R. W., Alison, D. \& Gilbert, P. Leading Articles. Br. Med. J. 1, 903-907 (1988).

25. Brown, S. P., Cornforth, D. M. \& Mideo, N. Evolution of virulence in opportunistic pathogens: generalism, plasticity, and control. Trends Microbiol. 20, 336-42 (2012).

26. Bryan, L. E. \& Kwan, S. Roles of ribosomal binding, membrane potential, and electron transport in bacterial uptake of streptomycin and gentamicin. Antimicrob. Agent Chemother. 23 (6), (1983).

27. Bryers, J. D. NIH Public Access. Artif. Cells Blood Substitutes Immobil. Biotechnol. Cells Blood Substit Immobi 100, 1-18 (2008).

28. Buchholz, F., Wolf, A., Lerchner, J., Mertens, F., Harms, H. \& Maskow, T. Chip calorimetry for fast and reliable evaluation of bactericidal and bacteriostatic treatments of biofilms. Antimicrob. Agents Chemother. 54, 312-319 (2010).

29. Busalmen, J. P., Sánchez, S. R. De \& Sa, S. R. De. Electrochemical Polarization-Induced Changes in the Growth of Individual Cells and Biofilms of Pseudomonas fluorescens Electrochemical Polarization-Induced Changes in the Growth of Individual Cells and Biofilms of Pseudomonas fluorescens. Society 71, 6235-6240 (2005).

30. Ceri, H., Olson, M. E., Stremick, C., Read, R. R., Morck, D. \& Buret, A. The Calgary Biofilm Device : New Technology for Rapid Determination of Antibiotic Susceptibilities of Bacterial Biofilms The Calgary Biofilm Device : New Technology for Rapid Determination of Antibiotic Susceptibilities of Bacterial Biofilms. J. Clin. Microbiol. 37, 1771 (1999).

31. Chalkley, L. J. \& Koornhof, H. J. Antimicrobial activity of ciprofloxacin against Pseudomonas aeruginosa, Escherichia coli, and Staphylococcus aureus determined by the killing curve method: Antibiotic comparisons and synergistic interactions. Antimicrob. Agents Chemother. 28, 331-342 (1985).

32. Chiew, Y. F., Yeo, S. F., Hall, L. M. C. \& Livermore, D. M. Can susceptibility to an antimicrobial be restored by halting its use? The case of streptomycin versus Enterobacteriaceae. J. Antimicrob. Chemother. 41, 247-251 (1998).

33. Chiou, C. S. \& Jones, A. L. Expression and identification of the strA-strB gene pair from streptomycin-resistant Erwinia amylovora. Gene 152, 47-51 (1995). 
34. Chopra, S., Harjai, K. \& Chhibber, S. Antibiotic susceptibility of ica-positive and icanegative MRSA in different phases of biofilm growth. J. Antibiot. (Tokyo). 68, 15-22 (2015).

35. Clark, D. J. \& Maaløe, O. DNA replication and the division cycle in Escherichia coli. J. Mol. Biol. 23, 99-112 (1967).

36. Clarke, E. G. C. Clarke's Isolation and Identification of Drugs in Pharmaceuticals, Body Fluids, and Post-mortem Material. Vol. 2. The Pharmaceutical Press, 17 Bloomsbury Square, London WC1A2NN., 1975.

37. Collier, D.N.; Hager, P.W., Phibbs, P. V. J. Catabolite repression control in the Pseudomonads. Res. Microbiol. 147, 551-561 (1996).

38. Conlon, B. P., Nakayasu, E. S., Fleck, L. E., LaFleur, M. D., Isabella, V. M., Coleman, K., Leonard, S. N., Smith, R. D., Adkins, J. N. \& Lewis, K. Activated ClpP kills persisters and eradicates a chronic biofilm infection. Nature 503, 365-70 (2013).

39. Conrad, R. S., Wulf, R. G. \& Clay, D. L. Effects of carbon sources on antibiotic resistance in Pseudomonas aeruginosa. Antimicrob. Agents Chemother. 15, 59-66 (1979).

40. Costerton, J. W.; Stewart, Philip S.; Greenberg, E. P. Bacterial Biofilms: A Common Cause of Persistent Infections. Science (80-. ). 284, 1318 - 1322 (1999).

41. Cotsonas King, A. \& Wu, L. Macromolecular synthesis and membrane perturbation assays for mechanisms of action studies of antimicrobial agents. Curr. Protoc.

Pharmacol. Chapter 13, 13A-7 (2009).

42. Crossman, L. C. Gould, V. C., Dow, J. M., Vernikos, G. S., Okazaki, A., Sebaihia, M., Saunders, D., Arrowsmith, C., Carver, T., Peters, N., Adlem, E., Kerhornou, A., Lord, A., Murphy, L., Seeger, K., Squares, R., Rutter, S., Quail, M. A., Rajandream, M.-A., Harris, D., Churcher, C., Bentley, S. D., Parkhill, J., Thomson, N. R. \& Avison, M. B. The complete genome, comparative and functional analysis of Stenotrophomonas maltophilia reveals an organism heavily shielded by drug resistance determinants. Genome Biol. 9, R74 (2008).

43. Culebras, E., Martínez, J. L., Baquero, F. \& Pérez-Díaz, J. C. pH modulation of aminoglycoside resistance in Staphylococcus epidermidis harbouring 6'-Naminoglycoside acetyltransferase. J. Antimicrob. Chemother. 37, 881-889 (1996). 
44. Dalhoff, A. The 'glucose effect' on growth inhibition of Echerichia coli by streptomycin, trimethoprim, and sulfamethoxazole. FEMS Microbiol. Lett. 6, 123-127 (1979).

45. Damper, P. \& Epstein, W. Role of the membrane potential in bacterial resistance to aminoglycoside antibiotics. Antimicrob. Agents Chemother. 20, 803-808 (1981).

46. Davenport, E. K., Call, D. R. \& Beyenal, H. Differential protection from tobramycin by extracellular polymeric substances from Acinetobacter baumannii and Staphylococcus aureus biofilms. Antimicrob. Agents Chemother. 58, 4755-61 (2014).

47. Davey, M. E. \& O’Toole, G. A. Microbial biofilms: from ecology to molecular genetics. Microbiol. Mol. Biol. Rev. 64, 847-67 (2000).

48. Davies, J; Davis, B. D. Code Words Induced. J. Biol. Chem. 243, 3312-3316 (1968).

49. De Groote, V. N. Verstraeten, N., Fauvart, M., Kint, C. I., Verbeeck, A. M., Beullens, S., Cornelis, P. \&Michiels, J. Novel persistence genes in Pseudomonas aeruginosa identified by high-throughput screening. FEMS Microbiol. Lett. 297, 73-9 (2009).

50. De la Fuente-Núñez, C., Reffuveille, F., Fernández, L. \& Hancock, R. E. W. Bacterial biofilm development as a multicellular adaptation: Antibiotic resistance and new therapeutic strategies. Curr. Opin. Microbiol. 16, 580-589 (2013).

51. De Lima Procópio, R. E., da Silva, I. R., Martins, M. K., de Azevedo, J. L. \& de Araújo, J. M. Antibiotics produced by Streptomyces. Brazilian J. Infect. Dis. 16, 466-471 (2012).

52. Denton, M. \& Kerr, K. G. Microbiological and clinical aspects of infection associated with Stenotrophomonas maltophilia. Clin. Microbiol. Rev. 11, 57-80 (1998).

53. Donlan, R. M. Biofilms: Microbial life on surfaces. Emerg. Infect. Dis. 8, 881-890 (2002).

54. Donlan, R. M. \& Costerton, J. W. Biofilms: survival mechanisms of clinically relevant microorganisms. Clin. Microbiol. Rev. 15, 167-93 (2002).

55. Dorrer, E. \& Teuber, M. Induction of polymyxin resistance in Pseudomonas fluorescens by Phosphate Limitation. Arch. Microbiol. 89, 87-89 (1977).

56. Dowd, S. E., Sun, Y., Secor, P. R., Rhoads, D. D., Wolcott, B. M., James, G. A. \& Wolcott, R.D Survey of bacterial diversity in chronic wounds using pyrosequencing, DGGE, and full ribosome shotgun sequencing. BMC Microbiol. 8, 43 (2008). 
57. Dri, A. M. \& Moreau, P. L. Control of the LexA regulon by pH: Evidence for a reversible inactivation of the LexA repressor during the growth cycle of Escherichia coli. Mol. Microbiol. 12, 621-629 (1994).

58. Dryden, M. S., Cooke, J. \& Davey, P. Antibiotic stewardship--more education and regulation not more availability? J. Antimicrob. Chemother. 64, 885-8 (2009).

59. Edwards, D. J. \& Holt, K. E. Beginner's guide to comparative bacterial genome analysis using next-generation sequence data. Microb. Inform. Exp. 3, 2 (2013).

60. Eisenberg, E. S., Mandel, L. J., Kaback, H. R. \& Miller, M. H. Quantitative association between electrical potential across the cytoplasmic membrane and early gentamicin uptake and killing in Staphylococcus aureus. J. Bacteriol. 157, 863-867 (1984).

61. Elkins, J. G., Hassett, D. J., Stewart, P. S., Schweizer, H. P. \& McDermott, T. R. Protective role of catalase in Pseudomonas aeruginosa biofilm resistance to hydrogen peroxide. Appl. Environ. Microbiol. 65, 4594-4600 (1999).

62. Eng, R. H. K., Padberg, F. T., Smith, S. M., Tan, E. N. \& Cherubin, C. E. Bactericidal Effects of Antibiotics. Spring 35, 1824-1828 (1991).

63. Felle, H., Porter, J. S., Slayman, C. L. \& Kaback, H. R. Quantitative measurements of membrane potential in Escherichia coli. Biochemistry 19, 3585-3590 (1980).

64. Fernández, L. \& Hancock, R. E. W. Adaptive and mutational resistance: Role of porins and efflux pumps in drug resistance. Clin. Microbiol. Rev. 25, 661-681 (2012).

65. Fijitani, Shigeki; Moffett, Katheryn S.; Yu, V. L. Pseudomonas aeruginosa. Infectious Disease and Antimicrobial Agents at <http://www.antimicrobe.org/new/b112.asp>

66. Flint, H. J., Duncan, S. H., Scott, K. P., Louis, P. Interactions and competition within the microbial community of the human colon: links between diet and health. Environ. Microbiol. 9 5:1101-1111(2007).

67. Forge, A. \& Schacht, J. Aminoglycoside antibiotics. Audiol. Neurootol. 5, 3-22 (2000).

68. Fraimow, H. S., Greenman, J. B., Leviton, I. M., Dougherty, T. J. \& Miller, M. H. Tobramycin uptake in Escherichia coli is driven by either electrical potential or ATP. $J$. Bacteriol. 173, 2800-2808 (1991).

69. Fraud, S. \& Poole, K. Oxidative stress induction of the MexXY multidrug efflux genes and promotion of aminoglycoside resistance development in Pseudomonas aeruginosa. Antimicrob. Agents Chemother. 55, 1068-1074 (2011). 
70. Frias-Lopez, J., Shi, Y., Tyson, G. W., Coleman, M. L., Schuster, S. C., Chisholm, S. W., \& DeLong, E. F. Microbial community gene expression in ocean surface waters. Proc. Natl. Acad. Sci. U. S. A. 105, 3805-10 (2008).

71. Fux, C. A., Costerton, J. W., Stewart, P. S. \& Stoodley, P. Survival strategies of infectious biofilms. Trends Microbiol. 13, 34-40 (2005).

72. Gabriel, P. S., Zhou, J., Tabibi, S., Chen, Y., Trauzzi, M., \& Saiman, L. Antimicrobial Susceptibility and Synergy Studies of Stenotrophomonas maltophilia Isolates from Patients with Cystic Fibrosis. Society 48, 168-171 (2004).

73. Geller, D. E. Aerosol antibiotics in CF. Respir. Care 54, 658-670 (2009).

74. Georgopapadakou, N. H. Penicillin-Binding Proteins and Bacterial Resistance. Antimicrob. Agents Chemother. 37, 2045-2053 (1993).

75. Ghadakpour, Mahtab; Bester, Elanna; Liss, Steven N.; Grdam, Michael; Droppo, Ian; Hota, S.; Wolfaardt, G. M. Integration and Proliferation of Pseudomonas aeruginosa PA01 in Multispecies Biofilms. Microbiol Ecol. 68, 121-131 (2014).

76. Giamarellou, H. \& Antoniadou, A. Antipseudomonal antibiotics. Med. Clin. North Am. 85, 19-42, v (2001).

77. Giamarellou, H. Prescribing guidelines for severe Pseudomonas infections. J. Antimicrob. Chemother. 49, 229-233 (2002).

78. Giaouris, E., Chorianopoulos, N., Doulgeraki, A. \& Nychas, G.-J. Co-culture with Listeria monocytogenes within a dual-species biofilm community strongly increases resistance of Pseudomonas putida to benzalkonium chloride. PLoS One 8, e77276 (2013).

79. Girard, L. P., Ceri, H., Gibb, A. P., Olson, M., \& Sepandj, F. MIC Versus MBEC to Determine the Antibiotic Sensitivity of Staphylococcus aureus in Peritoneal Dialysis Peritonitis. Peritoneal Dialysis International 652-656 (2010).

80. Gilbert, P., Allison, D. G. \& McBain, A. J. Biofilms in vitro and in vivo: do singular mechanisms imply cross-resistance? Symp. Ser. Soc. Appl. Microbiol. 98S-110S (2002).

81. Giwercman, B., Jensen, E. T. \& Kharazmi, a. Induction of Beta-Lactamase Production in Pseudomonas aeruginosa Biofilm. Antimicrob. Agents Chemother. 35, 1008-1010 (1991).

82. Gonzalez III, Uis S.; Spencer, J. P. Aminoglycosides: A Practical Review. Am. Fam. Physician 58, 1811-1820 (1998). 
83. Gordon, C. A., Hodges, N. A. \& Marriott, C. Antibiotic interaction and diffusion through alginate and exopolysaccharide of cystic fibrosis-derived Pseudomonas aeruginosa. $J$. Antimicrob. Chemother. 22, 667-674 (1988).

84. Goss, C. H., Otto, K., Aitken, M. L. \& Rubenfeld, G. D. Detecting Stenotrophomonas maltophilia does not reduce survival of patients with cystic fibrosis. Am. J. Respir. Crit. Care Med. 166, 356-61 (2002).

85. Gottenboss, B., van der Mei, H. C., Busscher, H. J. Models for studying initial adhesion and surface growth in biofilm formation on surfaces. Methods Enzymol. 310, 253-33 (1999).

86. Govan, J. R. W., Brown, A. R. \& Jones, A. M. Evolving epidemiology of Pseudomonas aeruginosa and the Burkholderia cepacia complex in cystic fibrosis lung infection. Future Microbiol. 2, 153-164 (2007).

87. Gristina, A. G., Hobgood, C. D., Webb, L. X. \& Myrvik, Q. N. Adhesive colonization of biomaterials and antibiotic resistance. Biomaterials 8, 423-426 (1987).

88. Gudmundsson, A., Erlendsdottir, H., Gottfredsson, M. \& Gudmundsson, S. Impact of pH and cationic supplementation on in vitro postantibiotic effect. Antimicrob. Agents Chemother. 35, 2617-24 (1991).

89. Hächler, H., Santanam, P. \& Kayser, F. H. Sequence and characterization of a novel chromosomal aminoglycoside phosphotransferase gene, aph (3')-IIb, in Pseudomonas aeruginosa. Antimicrob. Agents Chemother. 40, 1254-1256 (1996).

90. Hall-Stoodley, L., Costerton, J. W. \& Stoodley, P. Bacterial biofilms: from the natural environment to infectious diseases. Nat. Rev. Microbiol. 2, 95-108 (2004).

91. Hall-Stoodley, L. \& Stoodley, P. Evolving concepts in biofilm infections. Cell. Microbiol. 11, 1034-1043 (2009).

92. Han, H., Nam, H., Koh, Y., Hur, J. \& Jung, J. Molecular bases of high-level streptomycin resistance in Pseudomonas marginalis and Pseudomonas syringae pv. actinidiae. $J$. Microbiol. 41, 16-21 (2003).

93. Hancock, R. Uptake of 14C-streptomycin by some microorganisms and its relation to their streptomycin sensitivity. J. Gen. Microbiol. 28, 493-501 (1962). 
94. Hancock, R. E. Aminoglycoside uptake and mode of action-with special reference to streptomycin and gentamicin. II. Effects of aminoglycosides on cells. J. Antimicrob. Chemother. 8, 429-445 (1981).

95. Hancock, R. E., Raffle, V. J. \& Nicas, T. I. Involvement of the outer membrane in gentamicin and streptomycin uptake and killing in Pseudomonas aeruginosa. Antimicrob. Agents Chemother. 19, 777-85 (1981).

96. Hancock, R. E. W. \& Brinkman, F. S. L. Function of pseudomonas porins in uptake and efflux. Annu. Rev. Microbiol. 56, 17-38 (2002).

97. Hanlon, G. W. Bacteriophages: an appraisal of their role in the treatment of bacterial infections. Int. J. Antimicrob. Agents 30, 118-28 (2007).

98. Hansen, M. C., Tolker-Nielsen, T., Givskov, M. \& Molin, S. Biased 16S rDNA PCR amplification caused by interference from DNA flanking the template region. FEMS Microbiol. Ecol. 26, 141-149 (1998).

99. Harrison, J. J., Turner, R. J. \& Ceri, H. Persister cells, the biofilm matrix and tolerance to metal cations in biofilm and planktonic Pseudomonas aeruginosa. Environ. Microbiol. 7, 981-994 (2005).

100. Hentzer, M., Riedel, K., Rasmussen, T. B., Heydorn, A., Andersen, J. B., Parsek, M. R., Rice, S. A., Eberl, L., Molin, S., Høiby, N., Kjelleberg, S. \& Givskov, M. Inhibition of quorum sensing in Pseudomonas aeruginosa biofilm bacteria by a halogenated furanone compound. Microbiology 148, 87-102 (2002).

101. Hentzer, M., Teitzel, G. M., Balzer, G. J., Molin, S., Givskov, M., Matthew, R., Heydorn, A. \& Parsek, M. R. Alginate Overproduction Affects Pseudomonas aeruginosa Biofilm Structure and Function Alginate Overproduction Affects Pseudomonas aeruginosa Biofilm Structure and Function. J. Bacteriol. 183, 5395-5401 (2001).

102. Heydorn, A., Nielsen, A. T., Hentzer, M., Sternberg, C., Givskov, M., Ersboll, B. K. \& Molin, S. Quantification of biofilm structures by the novel computer program COMSTAT. Microbiology 146, 2395-2407 (2000).

103. Hoa, B. T. Q., Mahakarnchanakul, W., Sajjaanantakul, T. \& Kitpreechavanich, V. Efficacy of Sanitizers on Listeria, Salmonella, and Pseudomonas Single and Mixed Biofilms in a Seafood Processing Environment. 5, (2015). 
104. Hoffman, L. R., D'Argenio, D. A., MacCoss, M. J., Zhang, Z.. Jones, R. A. \& Miller, S. I. Aminoglycoside antibiotics induce bacterial biofilm formation. Nature 436, 1171-1175 (2005).

105. Hooper, D. C. Emerging mechanisms of fluoroquinolone resistance. Drug Resist. Updat. 2, 38-55 (2001).

106. Islam, A. Li, S. S., Oykhman, P., Timm-McCann, M., Huston, S. M., Stack, D., Xiang, R. F., Kelly, M. M. \& Mody, C. H. An Acidic Microenvironment Increases NK Cell Killing of Cryptococcus neoformans and Cryptococcus gattii by Enhancing Perforin Degranulation. PLoS Pathog. 9, (2013).

107. Ito, A., Taniuchi, A., May, T., Kawata, K. \& Okabe, S. Increased antibiotic resistance of Escherichia coli in mature biofilms. Appl. Environ. Microbiol. 75, 4093-100 (2009).

108. Jahoor, A., Patel, R., Bryan, A., Do, C., Krier, J., Watters, C., Wahli, W., Li, G., Williams, S. C. \& Rumbaugh, K. P. Peroxisome proliferator-activated receptors mediate host cell proinflammatory responses to Pseudomonas aeruginosa autoinducer. $J$. Bacteriol. 190, 4408-4415 (2008).

109. K. C., Iregbu; S.O., E. Pseudomonas aeruginosa infections in a tertiary hospital in Nigeria. African J. Clin. Exp. Microbiol. 16, 33-36 (2015).

110. Kadurugamuwa, J. L., Clarke, a. J. \& Beveridge, T. J. Surface action of gentamicin on Pseudomonas aeruginosa. J. Bacteriol. 175, 5798-5805 (1993).

111. Kanehisa, M. \& Goto, S. KEGG: kyoto encyclopedia of genes and genomes. Nucleic Acids Res. 28, 27-30 (2000).

112. Kataoka, D. The indirect pathogenicity of Stenotrophomonas maltophilia. Int. J. Antimicrob. Agents 22, 601 - 606 (2003).

113. Keren, I., Shah, D., Spoering, A., Kaldalu, N. \& Lewis, K. Specialized Persister Cells and the Mechanism of Multidrug Tolerance in Escherichia coli. 186, 8172-8180 (2004).

114. Khan, S. Proton chemical potential, proton electrical potential and bacterial motility. J. Mol. Biol. 138, 599 - 614 (1980).

115. De Kievit, T. R., Parkins, M. D., Gillia, R. J., Srikumar, R., Ceri, H., Poole, K., Iglewski, B. H., \& Storey, D. G. Multidrug Efflux Pumps : Expression Patterns and 
Contribution to Antibiotic Resistance in Pseudomonas aeruginosa Biofilms. Antimicrob. Agents Chemother. 45, 1761-1770 (2001).

116. Kingston, W. Streptomycin, Schatz v. Waksman, and the balance of credit for discovery. J. Hist. Med. Allied Sci. 59, 441-462 (2004).

117. Kitao, T., Miyoshi-Akiyama, T. \& Kirikae, T. AAC(6')-Iaf, a novel aminoglycoside 6'-N-acetyltransferase from multidrug-resistant Pseudomonas aeruginosa clinical isolates. Antimicrob. Agents Chemother. 53, 2327-2334 (2009).

118. Koch, C. \& Hoiby, N. Pathogenesis of cystic fibrosis. Lancet 341, 1065-1069 (1993).

119. Kroukamp, M. $\mathrm{CO}_{2}$ Measured in the Gas Phase as an indicator of Biofilm mtabolism. (Ryerson University, 2010).

120. Kroukamp, O., Dumitrache, R. G. \& Wolfaardt, G. M. Pronounced effect of the nature of the inoculum on biofilm development in flow systems. Appl. Environ. Microbiol. 76, 6025-6031 (2010).

121. Kroukamp, O. \& Wolfaardt, G. M. CO2 production as an indicator of biofilm metabolism. Appl. Environ. Microbiol. 75, 4391-4397 (2009).

122. Kukavica-Ibrulj, I. Bragonzi, A., Paroni, M., Winstanley, C., Sanschagrin, F., O'Toole, G. A. \& Levesque, R. C. In vivo growth of Pseudomonas aeruginosa strains PAO1 and PA14 and the hypervirulent strain LESB58 in a rat model of chronic lung infection. J. Bacteriol. 190, 2804-13 (2008).

123. Kumon, H., Tomochika, K., Matunaga, T., Ogawa, M. \& Ohmori, H. A sandwich cup method for the penetration assay of antimicrobial agents through Pseudomonas exopolysaccharides. Microbiol. Immunol. 38, 615-619 (1994).

124. Kureishi, A., Diver, J. M., Beckthold, B., Schollaardt, T. \& Bryan, L. E. Cloning and nucleotide sequence of Pseudomonas aeruginosa DNA gyrase gyrA gene from strain PAO1 and quinolone-resistant clinical isolates. Antimicrob. Agents Chemother. 38, 19441952 (1994).

125. Lambert, P. A. Mechanisms of antibiotic resistance in Pseudomonas aeruginosa. J. R. Soc. Med. 95 Suppl 4, 22-26 (2002).

126. Lavelle, P., Decaëns, T., Aubert, M., Barot, S., Blouin, M., Bureau, F., Margerie, P., Mora, P., Rossi, J.-P. Soil invertebrates and ecosystem services. Eur. J. Soil Biol. 42, S3-S15 (2006). 
127. Lerman, L. S. Acridine Mutagens and Dna Structure. J. Cell. Physiol. 64, SUPPL $1: 1-18$ (1964).

128. Levings, R. S., Partridge, S. R., Lightfoot, D., Hall, R. M. \& Djordjevic, S. P.

New integron-associated gene cassette encoding a 3-N-aminoglycoside acetyltransferase. Antimicrob. Agents Chemother. 49, 1238-1241 (2005).

129. Lewis, K. Riddle of Biofilm Resistance. Antimicrob. Agent Chemother. 45, 9991007 (2001).

130. Lewis, K. Persister cells. Annu. Rev. Microbiol. 64, 357-372 (2010).

131. Lewis, K. Persister cells, dormancy and infectious disease. Nat. Rev. Microbiol. 5, $48-56$ (2007).

132. Lindsay, D. \& von Holy, A. Bacterial biofilms within the clinical setting: what healthcare professionals should know. J. Hosp. Infect. 64, 313-325 (2006).

133. Lister, P. D., Wolter, D. J. \& Hanson, N. D. Antibacterial-resistant Pseudomonas aeruginosa: Clinical impact and complex regulation of chromosomally encoded resistance mechanisms. Clin. Microbiol. Rev. 22, 582-610 (2009).

134. Lomovskaya, O. Warren, M. S., Lee, A., Galazzo, J., Fronko, R., Lee, M., BLais, J., Cho, D., Chamberland, S., Renau, T., Leger, R., Hecker, S., Watkins, W., Hoshino, K., Ishida, H. \& Lee, V. J. Identification and Characterization of Inhibitors of Multidrug Resistance Efflux Pumps in Pseudomonas aeruginosa : Novel Agents for Combination Therapy Identification and Characterization of Inhibitors of Multidrug Resistance Efflux Pumps in Pseudomonas a. Antimicrob. Agents Chemother. 45, 105-116 (2001).

135. Madsen, J. S., Burmølle, M., Hansen, L. H. \& Sørensen, S. J. The interconnection between biofilm formation and horizontal gene transfer. FEMS Immunol. Med. Microbiol. 65, 183-95 (2012).

136. Mah, T. F. C. \& O’Toole, G. A. Mechanisms of biofilm resistance to antimicrobial agents. Trends Microbiol. 9, 34-39 (2001).

137. Mariam, S. H., Werngren, J., Aronsson, J., Hoffner, S. \& Andersson, D. I. Dynamics of antibiotic resistant mycobacterium tuberculosis during long-term infection and antibiotic treatment. PLoS One 6, (2011). 
138. Marks, L. R., Davidson, B. A., Knight, P. R. \& Hakansson, A. P. Interkingdom signaling induces Streptococcus pneumoniae biofilm dispersion and transition from asymptomatic colonization to disease. MBio 4, e00438-13-(2013).

139. Martin, Karen W.; Ernst, E. Herbal medicines for treatment of bacterial infections: a review of controlled clinical trials. J. Antimicrob. Chemother. 51, 241-246 (2003).

140. Mates, S. M., Eisenberg, E. S., Mandel, L. J., Patel, L., Kaback, H. R. \& Miller, M. H. Membrane potential and gentamicin uptake in Staphylococcus aureus. Proc. Natl. Acad. Sci. U. S. A. 79, 6693-6697 (1982).

141. McLarnon, Stuart J.; Holden, Darren; Ward, Donald T.; Jones, Malcolm N.; Elliot, Austin C.; Riccardi, D. Aminoglycoside antibiotics induce pH-sensitive activation of the calcium-sensing receptor. Biochem. Biophys. Res. Commun. 297, 71 - 77 (2002).

142. Meyer, F., Paarmann, D., D’Souza, M., Olson, R., Glass, E. M., Kubal, M., Paczian, T., Rodriguez, A., Stevens, R., Wilke, A., Wilkening, J.\& Edwards, R. A.. The metagenomics RAST server - a public resource for the automatic phylo- genetic and functional analysis of metagenomes. BMC Bioinformatics 9, 386 (2008).

143. Milman, N. \& Dahlager, J. The pH-dependent influence of aminoglycoside antibiotics on iodohippurate accumulation in rabbit renal cortical slices. J. Antibiot. (Tokyo). 31, 1183-1187 (1978).

144. Mingeot-Leclercq, M. P., Glupczynski, Y. \& Tulkens, P. M. Aminoglycosides: Activity and resistance. Antimicrob. Agents Chemother. 43, 727-737 (1999).

145. Mogayzel, P. J., Naureckas, E. T., Robinson, K. A., Mueller, G., Hadjiliadis, D., Hoag, J. B., Lubsch, L., Hazel, L., Sabadosa, K. \& Marshall, B.Cystic fibrosis pulmonary guidelines: Chronic medications for maintenance of lung health. Am. J. Respir. Crit. Care Med. 187, 680-689 (2013).

146. Möker, N., Dean, C. R. \& Tao, J. Pseudomonas aeruginosa increases formation of multidrug-tolerant persister cells in response to quorum-sensing signaling molecules. $J$. Bacteriol. 192, 1946-55 (2010).

147. Molin, Søren; Tolker-Nielsen, T. Gene transfer occurs with enhanced efficiency in biofilms and induces enhanced stabilisation of the biofilm structure. Curr. Opin.

Biotechnol. 14, 255 - 261 (2003). 
148. Moore, N. M., Flaws, M. L., Flaws, M. L., Si, S. M. A. \& Univer-, R.

Antimicrobial Resistance Mechanisms in Pseudomonas aeruginosa difficult to treat because there are limited drugs with Focus on Pseudomonas aeruginosa. 24, 47-52 (2011).

149. Morita, Y., Kimura, N., Mima, T., Mizushima, T. \& Tsuchiya, T. Roles of MexXY- and MexAB-multidrug efflux pumps in intrinsic multidrug resistance of Pseudomonas aeruginosa PAO1. J. Gen. Appl. Microbiol. 47, 27-32 (2001).

150. Mukkada, A. J., Long, G. L. \& Romano, A. H. The uptake of 2-deoxy-D-glucose by Pseudomonas aeruginosa and its regulation. Biochem. J. 132, 155-162 (1973).

151. Mulcahy, H., Charron-Mazenod, L. \& Lewenza, S. Extracellular DNA chelates cations and induces antibiotic resistance in Pseudomonas aeruginosa biofilms. PLoS Pathog. 4, (2008).

152. Musk, D. J., Banko, D. A. \& Hergenrother, P. J. Iron salts perturb biofilm formation and disrupt existing biofilms of Pseudomonas aeruginosa. Chem. Biol. 12, 789-796 (2005).

153. Nazik, H., Ongen, B., Erturan, Z. \& Salcioğlu, M. Genotype and antibiotic susceptibility patterns of Pseudomonas aeruginosa and Stenotrophomonas maltophilia isolated from cystic fibrosis patients. Jpn. J. Infect. Dis. 60, 82-6 (2007).

154. Ng, F. M. \& Dawes, E. A. Chemostat studies on the regulation of glucose metabolism in Pseudomonas aeruginosa by citrate. Biochem. J. 132, 129-40 (1973).

155. Nguyen, D. et al. Active starvation responses mediate antibiotic tolerance in biofilms and nutrient-limited bacteria. Science 334, 982-6 (2011).

156. Nickel, J. C., Ruseska, I., Wright, J. B. \& Costerton, J. W. Tobramycin resistance of Pseudomonas aeruginosa cells growing as a biofilm on urinary catheter material. Antimicrob. Agents Chemother. 27, 619-24 (1985).

157. Nithya, C., Begum, M. F. \& Pandian, S. K. Marine bacterial isolates inhibit biofilm formation and disrupt mature biofilms of Pseudomonas aeruginosa PAO1. Appl. Microbiol. Biotechnol. 88, 341-358 (2010).

158. O’May, C. Y., Reid, D. W. \& Kirov, S. M. Anaerobic culture conditions favor biofilm-like phenotypes in Pseudomonas aeruginosa isolates from patients with cystic fibrosis. FEMS Immunol. Med. Microbiol. 48, 373-380 (2006). 
159. Overbeek, R., Olson, R., Pusch, G. D., Olsen, G. J., Davis, J. J., Disz, T., Edwards, R. A., Gerdes, S., Parrello, B., Shukla, M., Vonstein, V., Wattman, A. R., Xia, F. \& Stevens, R. The SEED and the Rapid Annotation of microbial genomes using Subsystems Technology (RAST). Nucleic Acids Res. 42, 1-9 (2013).

160. Palmer, K. L., Aye, L. M. \& Whiteley, M. Nutritional cues control Pseudomonas aeruginosa multicellular behavior in cystic fibrosis sputum. J. Bacteriol. 189, 8079-8087 (2007).

161. Paulander, W., Maisnier-Patin, S. \& Andersson, D. I. The fitness cost of streptomycin resistance depends on rpsL mutation, carbon source and RpoS $(\sigma S)$. Genetics 183, 539-546 (2009).

162. Pedersen, S. S., Høiby, N., Espersen, F. \& Koch, C. Role of alginate in infection with mucoid Pseudomonas aeruginosa in cystic fibrosis. Thorax 47, 6-13 (1992).

163. Piddock, L. J. Fluoroquinolone resistance: overuse of fluoroquinolones in human and veterinary medicine can breed resistance. BMJ 317, 1029-1030 (1998).

164. Poole, K. Poole 2005. Aminoglycoside Resistance in Pseudomonas aeruginosa. Antimicrob. Agent Chemother. 42, 479-487 (2005).

165. Popowska, M. Rzeczycka, M., Miernik, A., Krawczyk-Balska, A., Walsh, F. \& Duffy, B. Influence of Soil Use on Prevalence of Tetracycline, Streptomycin, and Erythromycin Resistance and Associated Resistance Genes. Antimicrob. Agents Chemother. 56, 1434-1443 (2012).

166. Qureshi, N., Annous, B. A., Ezeji, T. C., Karcher, P., Maddox, I. S. Biofilm reactors for industrial bioconversion processes: employing potential of enhanced reaction rates. Microb. Cell Fact. 4 1:24 (2005).

167. Ramirez, M. S. \& Tolmasky, M. E. Aminoglycoside modifying enzymes. Drug Resist. Updat. 13, 151-171 (2010).

168. Reimmann, C., Beyeler, M., Latifi, A., Winteler, H., Foglino, M., Lazdunski, A., \& Haas, D. The global activator GacA of Pseudomonas aeruginosa PAO positively controls the production of the autoinducer N-butyryl-homoserine lactone and the formation of the virulence factors pyocyanin, cyanide, and lipase. Mol. Microbiol. 24, 309-319 (1997). 
169. Rojo, F. Carbon catabolite repression in Pseudomonas: Optimizing metabolic versatility and interactions with the environment. FEMS Microbiol. Rev. 34, 658-684 (2010).

170. Rölleke, S., Muyzer, G., Wawer, C., Wanner, G. \& Lubitz, W. Identification of bacteria in a biodegraded wall painting by denaturing gradient gel electrophoresis of PCR-amplified gene fragments coding for 16S rRNA. Appl. Environ. Microbiol. 62, 2059-65 (1996).

171. Ronan, E. Investigating Microbial Ecology at Solid-Air Interfaces. (Ryerson University, 2011). at <http://digital.library.ryerson.ca/islandora/object/RULA\%3A1157>

172. Rosenkranz, H. S. Macromolecular synthesis and the bactericidal effect of streptomycin. J. Bacteriol. 87, 606-8 (1964).

173. Rossolini, G. M. \& Mantengoli, E. Treatment and control of severe infections caused by multiresistant Pseudomonas aeruginosa. Clin. Microbiol. Infect. 11 Suppl 4, 17-32 (2005).

174. Ryan, R. P., Monchy, S., Cardinale, M., Taghavi, S., Crossman, L., Avison, M. B., Berg, G., van der Lelie, D. ,\& Dow, J. M. The versatility and adaptation of bacteria from the genus Stenotrophomonas. Nat. Rev. Microbiol. 7, 514-25 (2009).

175. Sader, H. S. Antimicrobial susceptibility of uncommonly isolated non-enteric Gram-negative bacilli. Int. J. Antimicrob. Agents 25, 95 - 109

176. Salipante, S. J., Sengupta, D. J., Rosenthal, C., Costa, G., Spangler, J., Sims, E. H., Jacobs, M. A., Miller, S. I., Hoogestraat, D. R., Cookson, B. T., McCoy, C., Matsen, F. A., Shendure, J., Lee, C. C., Harkins, T. T., \& Hoffman, N. G. Rapid 16S rRNA nextgeneration sequencing of polymicrobial clinical samples for diagnosis of complex bacterial infections. PLoS One 8, e65226 (2013).

177. Salter, S. J., Cox, M. J., Turek, E. M., Calus, S. T., Cookson, W. O., Moffatt, M. O., Turner, P., Parkhill, J., Loman, N. J., \& Walker. A. W. Reagent and laboratory contamination can critically impact sequence-based microbiome analyses. BMC Biol. 12, 87 (2014).

178. Scholz, P., Haring, V., Wittmann-Liebold, B. Ashman,K., Bagdasarian, M. \& Scherzinger, E. Complete nucleotide sequence and gene organization of the broad-hostrange plasmid RSF1010. Gene 75, 271-288 (1989). 
179. Shapiro, H. M. Membrane potential estimation by flow cytometry. Methods 21, $271-279$ (2000).

180. Shaw, K. J., Rather, P. N., Hare, R. S. \& Miller, G. H. Molecular genetics of aminoglycoside resistance genes and familial relationships of the aminoglycosidemodifying enzymes. Microbiol. Rev. 57, 138-163 (1993).

181. Simões, M., Simões, L. C. \& Vieira, M. J. Species association increases biofilm resistance to chemical and mechanical treatments. Water Res. 43, 229-37 (2009).

182. Spencer, R. C. The emergence of epidemic, multiple-antibiotic-resistant Stenotrophomonas (Xanthomonas) maltophilia and Burkholderia (Pseudomonas) cepacia. J. Hosp. Infect. 30 Suppl, 453-464 (1995).

183. Spoering, aA. L. \& Lewis, K. Biofilms and planktonic cells of Pseudomonas aeruginosa have similar resistance to killing by antimicrobials. J. Bacteriol. 183, 67466751 (2001).

184. Stanier, R. Y., Palleroni, N. J. \& Doudoroff, M. The aerobic Pseudomonads: a taxonomic study. J. Gen. Microbiol. 43, 159-271 (1966).

185. Starner, T. D. \& McCray Jr., P. B. Pathogenesis of early lung disease in cystic fibrosis: A window of opportunity to eradicate bacteria. Ann. Intern. Med. 143, 816-822 (2005).

186. Stepanyan, K., Wenseleers, T., Duéñez-Guzmán, E. A., Muratori, F., Van den Bergh, B., Verstraeten, N., De Meester, L., Verstrepen, K. J., Fauvart, M. \& Michiels, J. Fitness trade-offs explain low levels of persister cells in the opportunistic pathogen Pseudomonas aeruginosa. Mol. Ecol. n/a-n/a (2015). doi:10.1111/mec.13127

187. Stewart, P. S. Biofilm accumulation model that predicts antibiotic resistance of Pseudomonas aeruginosa biofilms. Antimicrob. Agents Chemother. 38, 1052-8 (1994).

188. Stewart, P. S. Mechanisms of antibiotic resistance in bacterial biofilms. Int. J. Med. Microbiol. 292, 107-113 (2002).

189. Stewart, P. S. \& Costerton, J. W. Antibiotic resistance of bacteria in biofilms. Lancet 358, 135-138 (2001).

190. Stiefel, P., Schmidt-Emrich, S., Maniura-Weber, K., Ren, Q. Critical aspects of using bacterial cell viability assays with the fluorophores SYTO9 and propidium iodide. BMC Microbiol. 15 1, 36 (2015). 
191. Stockwell, V. O. \& Duffy, B. Use of antibiotics in plant agriculture Fire blight: the primary use of antibiotics on plants activity and mechanisms of resistance in Erwinia amylovora. 31, 199-210 (2012).

192. Stover, C., Pham, X., Erwin, A. \& Mizoguchi, S. Complete genome sequence of Pseudomonas aeruginosa PAO1, an opportunistic pathogen. Nature 406, 959-964 (2000).

193. Sulakvelidze, A. Phage therapy: an attractive option for dealing with antibioticresistant bacterial infections. Drug Discov. Today 10, 807-9 (2005).

194. Taber, H. W., Mueller, J. P., Miller, P. F. \& Arrow, a S. Bacterial uptake of aminoglycoside antibiotics. Microbiol. Rev. 51, 439-457 (1987).

195. Taghavi, S., Garafola, C., Monchy, S., Newman, L., Hoffman, A., Weyens, N., Barac, T., Vangronsveld, J., \& van der Lelie, D. Genome survey and characterization of endophytic bacteria exhibiting a beneficial effect on growth and development of poplar trees. Appl. Environ. Microbiol. 75, 748-57 (2009).

196. Tate, S., MacGregor, G., Davis, M., Innes, J. A. \& Greening, A. P. Airways in cystic fibrosis are acidified: detection by exhaled breath condensate. Thorax 57, 926-929 (2002).

197. Tenover, F. C. Mechanisms of Antimicrobial Resistance in Bacteria. Am. J. Med. 119, (2006).

198. Toole, G. O., Kaplan, H. B. \& Kolter, R. B Iofilm F Ormation As M Icrobial. 4979 (2000).

199. Torsvik, V. \& Øvreås, L. Microbial diversity and function in soil: from genes to ecosystems. Curr. Opin. Microbiol. 5, 240-5 (2002).

200. Tringe, S. G. \& Rubin, E. M. Metagenomics: DNA sequencing of environmental samples. Nat. Rev. Genet. 6, 805-814 (2005).

201. Tseng, B. S., Zhang, W., Harrison, J. J., Quach, T. P., Song, J. L., Penterman, J., Singh, P. K., Chopp, D. L., Packman, A. I., Parsek, M. R. The extracellular matrix protects Pseudomonas aeruginosa biofilms by limiting the penetration of tobramycin. Environ. Microbiol. 15, 2865-78 (2013).

202. Tseng, C.-C., Fang, W.-F., Huang, K.-T., Chang, P.-W., Tu, M.-L., Shiang, Y.-P., Douglas, I. S., Lin, M.-C. Risk factors for mortality in patients with nosocomial 
Stenotrophomonas maltophilia pneumonia. Infect. Control Hosp. Epidemiol. 30, 11931202 (2009).

203. van Overbeek, L. S., Wellington, Elisabeth, M. H., Egan, S., Smalla, K., Heuer, H., Collard, J.-M., Guillaume, Gillian, Karagouni, A. D>, Nikolakopoulou, T. L., \& van Elsas, J. D. Prevalence of streptomycin-resistance genes in bacterial populations in European habitats. FEMS Microbiol. Ecol. 42, 277-288 (2002).

204. Vidaver, A. K. Uses of antimicrobials in plant agriculture. Clin. Infect. Dis. 34 Suppl 3, S107-S110 (2002).

205. Villagra, N. A., Fuentes, J. A., Jofré, M. R., Hidalgo, A. A., García, P., \& Mora, G. C.. The carbon source influences the efflux pump-mediated antimicrobial resistance in clinically important Gram-negative bacteria. J. Antimicrob. Chemother. 67, 921-927 (2012).

206. Webber, M. A. \& Piddock, L. J. V. The importance of efflux pumps in bacterial antibiotic resistance. J. Antimicrob. Chemother. 51, 9-11 (2003).

207. Weinstock, G. M. Genomics and bacterial pathogenesis. Emerg. Infect. Dis. 6, 496-504 (2000).

208. Whiting, P. H.; Midgley, M.; Dawes, E. A. The role of glucose limitation in the regulation of the transport of glucose, gluconate and 2-oxogluconate, and of glucose metabolism in Pseudomonas aeruginosa. J. Gen. Microbiol. 92, 304-310 (1976).

209. Whooley, M. A. \& McLoughlin, A. J. The protonmotive force in Pseudomonas aeruginosa and its relationship to exoprotease production. J. Gen. Microbiol. 129, 989996 (1983).

210. Wolcott, R. D., Rhoads, D. D. \& Dowd, S. E. Biofi lms and chronic wound inflammation. J. Wound Care 17, 333-341 (2008).

211. Wolfaardt, G. M., Lawrence, J. R., Robarts, R. D., Caldwell, S. J. \& Caldwell, D. E. Multicellular organization in a degradative biofilm community. Appl. Environ. Microbiol. 60, 434-446 (1994).

212. Wolfgang, M. C., Kulasekara, B. R., Liang, X., Boyd, D., Wu, K., Yang, Q., ... Lory, S. Conservation of genome content and virulence determinants among clinical and environmental isolates of Pseudomonas aeruginosa. Proc. Natl. Acad. Sci. U. S. A. 100, 8484-8489 (2003). 
213. Wozniak, D. J., Wyckoff, T. J. O., Starkey, M., Keyser, R., Azadi, P., O’Toole, G. a, \& Parsek, M. R. Alginate is not a significant component of the extracellular polysaccharide matrix of PA14 and PAO1 Pseudomonas aeruginosa biofilms. Proc. Natl. Acad. Sci. U. S. A. 100, 7907-7912 (2003).

214. Wu, K., Yau, Y. C. W., Matukas, L. \& Waters, V. Biofilm compared to conventional antimicrobial susceptibility of Stenotrophomonas maltophilia isolates from cystic fibrosis patients. Antimicrob. Agents Chemother. 57, 1546-1548 (2013).

215. Xiong, Y. Q., Caillon, J., Drugeon, H., Potel, G. \& Baron, D. Influence of pH on adaptive resistance of Pseudomonas aeruginosa to aminoglycosides and their postantibiotic effects. Antimicrob. Agents Chemother. 40, 35-39 (1996).

216. Yang, L., Liu, Y., Wu, H., Hóiby, N., Molin, S., \& Song, Z. Current understanding of multi-species biofilms. Int. J. Oral Sci. 3, 74-81 (2011).

217. Yeung, C. W., Woo, M., Lee, K. \& Greer, C. W. Characterization of the bacterial community structure of Sydney Tar Ponds sediment. Can. J. Microbiol. 57, 493-503 (2011).

218. Zerbino, D. R. Using the Velvet de novo assembler for short-read sequencing technologies. Curr. Protoc. Bioinformatics Chapter 11, Unit 11.5 (2010).

219. Zhao, Q., Li, X. Z., Srikumar, R. \& Poole, K. Contribution of outer membrane efflux protein OprM to antibiotic resistance in Pseudomonas aeruginosa independent of MexAB. Antimicrob. Agents Chemother. 42, 1682-8 (1998).

220. Zuroff, T. R., Bernstein, H., Lloyd-Randolfi, J., Jimenez-Taracido, L., Stewart, P. S., \& Carlson, R. P. Robustness analysis of culturing perturbations on Escherichia coli colony biofilm beta-lactam and aminoglycoside antibiotic tolerance. BMC Microbiol. 10, 185 (2010). 